\title{
DISCOVERIES FROM A NEAR-INFRARED PROPER MOTION SURVEY USING MULTI-EPOCH TWO MICRON ALL-SKY SURVEY DATA*
}

\author{
J. Davy Kirkpatrick ${ }^{1}$, Dagny L. Looper ${ }^{2}$, Adam J. Burgasser ${ }^{3}$, Steven D. Schurr ${ }^{4}$, Roc M. Cutri ${ }^{1}$, Michael C. Cushing ${ }^{5}$, \\ Kelle L. Cruz ${ }^{6}$, Anne C. Sweet ${ }^{7}$, Gillian R. Knapp ${ }^{8}$, Travis S. Barman ${ }^{9}$, John J. Bochanski ${ }^{10}$, Thomas L. Roellig ${ }^{11}$, \\ IAN S. MCLEAN ${ }^{12}$, MARK R. MCGovern ${ }^{13}$, AND EMily L. RicE ${ }^{14}$ \\ ${ }^{1}$ Infrared Processing and Analysis Center, MS 100-22, California Institute of Technology, Pasadena, CA 91125, USA; davy@ipac.caltech.edu \\ ${ }^{2}$ Institute for Astronomy, University of Hawaii, 2680 Woodlawn Drive, Honolulu, HI 96822, USA \\ ${ }^{3}$ Center for Astrophysics and Space Science, University of California, San Diego, CA 92093, USA \\ ${ }^{4}$ Planck Science Center, MS 220-6, California Institute of Technology, Pasadena, CA 91125, USA \\ 5 Jet Propulsion Laboratory, Pasadena, CA 91109, USA \\ ${ }^{6}$ Department of Physics and Astronomy, Hunter College, New York, NY 10065, USA \\ ${ }^{7}$ British Consulate, San Francisco, CA 94104, USA \\ ${ }^{8}$ Department of Astrophysical Sciences, Princeton University, Princeton, NJ 08544, USA \\ ${ }^{9}$ Lowell Observatory, 1400 West Mars Hill Road, Flagstaff, AZ 86001, USA \\ ${ }^{10}$ Massachusetts Institute of Technology, 77 Massachusetts Avenue, Building 37, Cambridge, MA 02139, USA \\ ${ }^{11}$ NASA Ames Research Center, MS 245-6, Moffett Field, CA 94035-1000, USA \\ 12 Department of Physics and Astronomy, UCLA, Los Angeles, CA 90095-1562, USA \\ ${ }^{13}$ Antelope Valley College, Lancaster, CA 93536, USA \\ ${ }^{14}$ American Museum of Natural History, New York, NY 10024, USA \\ Received 2009 December 26; accepted 2010 July 12; published 2010 August 27
}

\begin{abstract}
We have conducted a $4030 \mathrm{deg}^{2}$ near-infrared proper motion survey using multi-epoch data from the Two Micron All-Sky Survey (2MASS). We find 2778 proper motion candidates, 647 of which are not listed in SIMBAD. After comparison to Digitized Sky Survey images, we find that 107 of our proper motion candidates lack counterparts at $B, R$, and $I$ bands and are thus 2MASS-only detections. We present results of spectroscopic follow-up of 188 targets that include the infrared-only sources along with selected optical-counterpart sources with faint reduced proper motions or interesting colors. We also establish a set of near-infrared spectroscopic standards with which to anchor near-infrared classifications for our objects. Among the discoveries are six young field brown dwarfs, five "red L" dwarfs, three L-type subdwarfs, twelve M-type subdwarfs, eight "blue L" dwarfs, and several T dwarfs. We further refine the definitions of these exotic classes to aid future identification of similar objects. We examine their kinematics and find that both the "blue L" and "red L" dwarfs appear to be drawn from a relatively old population. This survey provides a glimpse of the kinds of research that will be possible through time-domain infrared projects such as the UKIDSS Large Area Survey, various VISTA surveys, and WISE, and also through $z$ - or $y$-band enabled, multi-epoch surveys such as Pan-STARRS and LSST.
\end{abstract}

Key words: brown dwarfs - infrared: stars - proper motions - solar neighborhood - stars: late-type

Online-only material: color figures, machine-readable table

\section{INTRODUCTION}

During the first quarter of the 20th century, cataloging the stellar constituents of the Solar Neighborhood became a major focus of astronomical research. Although the closest of the bright stars were identifiable through dedicated trigonometric parallax programs, such programs were not feasible for the wealth of dimmer objects. As a result the search for the faintest of the nearby stars came to rely heavily on the fact that tangential motion of the closest objects would be measurable against the sea of non-moving background objects. As a result, these proper motion measurements using multi-epoch photographic plates became the primary means of discovery.

The closest star to the Sun, Proxima Centauri, was discovered as a distant, common proper motion companion of $\alpha$ Centauri

\footnotetext{
* Some of the spectroscopic data presented herein were obtained at the W. M. Keck Observatory, which is operated as a scientific partnership among the California Institute of Technology, the University of California, and the National Aeronautics and Space Administration. The Observatory was made possible by the generous financial support of the W. M. Keck Foundation. Other spectroscopic data were collected at the Subaru Telescope, which is operated by the National Astronomical Observatory of Japan.
}

AB by Innes (1915). Barnard (1916) discovered the second closest system - most commonly known as Barnard's star-via its proper motion, which is still the highest known for any star (10'.37 $\mathrm{yr}^{-1}$; Benedict et al. 1999). Wolf (1919) used proper motion to uncover the third closest system, known as Wolf 359, and Ross (1926) did the same to reveal the seventh, eighth, and eleventh nearest systems, known as Ross 154, Ross 248 , and Ross 128, respectively. Taking this work to even fainter limits, van Biesbroeck $(1944,1961)$ performed a photographic search for companions to nearby stars and revealed two late-M dwarfs, known as van Biesbroeck 8 (vB 8) and van Biesbroeck 10 (vB 10). Deeper, larger scale proper motion surveys by Giclas et al. (1971, 1978) and by Luyten (1979a, 1979b, 1980a, 1980b) were performed in the mid-20th century. These Luyten references tabulate the results of all previous motion surveys done through the 1970s. These surveys accounted for a total of over 58,000 motion stars with $\mu>00^{\prime} .18 \mathrm{yr}^{-1}$ down to the $B$-band plate limit of the Palomar Observatory Sky Survey (POSS) and to brighter limits for stars south of the POSS itself.

A major limitation of these previous surveys was their reliance on optical data, primarily at $B$ and $R$ bands; a lack of deep, dual-epoch material in the far southern sky; and an 
inability to probe close to the Galactic plane. Recent largearea surveys have attempted to remove some of these biases while still using photographic plate material. Luyten's surveys, which generally required that objects be detectable on both the $B$ - and $R$-band plates and be located at relatively high galactic latitude, have now been supplemented with the LSPM/LSR proper motion survey of Lépine et al. $(2002,2003)$ and Lépine $(2005,2008)$, which uses two-epoch $R$-band data only (when two epochs are available) to push to intrinsically redder and fainter objects. Similar investigations, many concentrating more heavily on the less-complete southern sky, have been performed by the Wroblewski \& Torres survey (WT; Wroblewski \& Costa 2001 and references therein), the Calan European Southern Observatory survey (Calan-ESO; Ruiz et al. 2001), the Automated Plate Measuring Proper Motion survey (APMPM; Scholz et al. 2000), the SuperCOSMOS Sky Survey Proper Motion survey (SSSPM; Scholz \& Meusinger 2002), the Liverpool-Edinburgh High Proper Motion survey (LEHPM; Pokorny et al. 2004), and the SuperCOSMOSRECONS survey (SCR; Finch et al. 2007 and references therein).

CCD-based surveys have also begun to have an impact. The SkyMorph database from the Near Earth Asteroid Tracking program uncovered a previously unrecognized late-M dwarf moving at $5^{\prime \prime} .05 \mathrm{yr}^{-1}$ that now ranks as one of the closest stars to the Sun (Teegarden et al. 2003). This discovery highlights the fact that deeper visible-light surveys with shorter epoch differences will be able to detect nearby stars with motions too large to have been detected with prior searches. Surveys by the Panoramic Survey Telescope and Rapid Response System (Pan-STARRS; Kaiser 2004) in the northern hemisphere and Large Synoptic Survey Telescope (LSST; Ivezic et al. 2008) and SkyMapper Telescope (Southern Sky Survey, aka S3; Keller et al. 2007) in the southern hemisphere will provide an ultradeep CCD-based proper motion catalog for the entire sky.

Limiting the search to optical surveys will, of course, render the census very incomplete for the faintest, coolest targets that have their peak flux at longer wavelengths. As photometric searches of the Two Micron All-Sky Survey (2MASS), the Sloan Digital Sky Survey (SDSS), and the Deep Near-infrared Survey of the Southern Sky (DENIS) have shown, the Solar Neighborhood is replete with, and perhaps even dominated by, dwarfs cooler than type M. Most of these objects have been uncovered using color selections appropriate for cool dwarfs with solar age and solar metallicity, but this method will miss cool halo objects with very low metallicity and any other objects of unusual color. Therefore, proper motion surveys at these longer wavelengths are needed to search for classes of very cool, nearby objects that may have heretofore gone unnoticed.

With this point in mind, researchers have begun to perform proper motion surveys beyond $1 \mu \mathrm{m}$. The Southern Infrared Proper Motion Survey (SIPS; Deacon \& Hambly 2007) compares $I$-band SuperCOSMOS data to 2 MASS $J, H$, and $K_{s}$ data, although in this case objects are chosen using 2MASS colors before being paired up with SuperCOSMOS I-band data, hence leading to some of the same biases and incompletenesses noted above. Sheppard \& Cushing (2009) have performed a proper motion search free of these photometric-selection biases by performing a cross-matching of 2MASS $\left(J, H, K_{s}\right)$ with SDSS Data Releases 1 through $5(u, g, r, i, z)$. This work uncovered 36 objects with $0^{\prime \prime} 2 \mathrm{yr}^{-1}<\mu<1^{\prime \prime} .0 \mathrm{yr}^{-1}$ that were not cataloged previously. Six of these have been followed up spectroscopically and the results include one mid-T dwarf, a late-M subdwarf, and an unusually blue L dwarf. Even more recently, Deacon et al. (2009) have matched up data from the United Kingdom Infrared Deep Sky Survey (UKIDSS; Lawrence et al. 2007) Data Release 4 with 2MASS to identify 267 low-mass stars and brown dwarfs via their proper motions; of these, ten known $\mathrm{L}$ dwarfs and one known $\mathrm{T}$ dwarf were recovered and nine new $\mathrm{L}$ dwarf candidates were identified.

We present here a large-area $\left(\sim 4030 \mathrm{deg}^{2}\right.$, or $\sim 10 \%$ of the sky) proper motion survey where both epochs of coverage lie longward of $1 \mu \mathrm{m}$. By performing a color-free proper motion search we can achieve several goals. (1) We can determine the location of unusual objects in color space to enable future photometric searches for these objects. (2) The kinematic bias present in a proper motion survey will enable us to find very old brown dwarfs, and such newfound discoveries will help to expand the list of observed low-metallicity, low-temperature atmospheres and provide invaluable tests of atmospheric theory. (3) We can begin to explore the mass function of substellar halo objects, allowing us for the first time to probe the efficiency of brown dwarf formation in low-metallicity environments.

\section{SURVEY METHODOLOGY}

\subsection{The 1X-6X Survey (List 1)}

For the first part of our survey we used data from the 2MASS All-Sky Point Source Catalog (PSC) as epoch 1 and from the 2MASS 6X Point Source Working Database/Catalog as epoch 2. The latter catalog comprises a set of special observations made at the end of 2MASS operations and taken with exposures six times longer than the main survey. The area covered by the $6 \mathrm{X}$ observations and hence the total area overlapped by both data sets is $\sim 580 \mathrm{deg}^{2}$. The epoch difference is 1-3 years. See Figure 1 for a graphical representation of the $6 \mathrm{X}$ coverage of the sky.

For each of the $1376 \mathrm{X}$ nights, a file was produced during 2MASS 6X data processing that listed all of the objects in the $6 \mathrm{X}$ scan that failed to match a source within a 0.5 search radius in the 2MASS $1 \mathrm{X}$ data. This resulted in 824,383 missing sources. After removing objects that were within $10^{\prime \prime}$ of the $6 \mathrm{X}$ scan edges, objects identified as known minor planets, objects that were confused with other detections in the scan, and sources of less than ideal photometric quality (i.e., sources for which none of the bands had photometric quality ph_qual $=\mathrm{A}$ ), the source list was reduced to 1181 sources. All of these were checked for additional problems by looking at side-by-side images of the field from both 2MASS and the Digital Sky Surveys (DSS) ${ }^{15}$. Problems identified at this stage were usually false motions induced by source confusion or by extended morphology.

After these visual checks, a total of 359 confirmed sources remained, most of which are clearly visible on the DSS plates. $185(51.5 \%)$ of the sources are not listed in SIMBAD, and 29 $(8.1 \%)$ have no counterpart within a 3".0 radius in the USNO-B Catalog (Monet et al. 2003). Of these 29, 8 (2.2\% of the original sample) are 2MASS-only sources because they have no visible counterpart on the DSS plates once motion and faint counterparts (such as I-only sources, not included in the USNO-B Catalog) are accounted for. All eight of these infrared-only discoveries lie in front of or in the immediate vicinity of the Large and Small Magellanic Clouds (LMC, SMC). This is no surprise since the LMC and SMC were the two largest targets of the 2MASS 6X observing campaign (see Figure 1).

\footnotetext{
15 See http://archive.stsci.edu/dss/sites.html.
} 


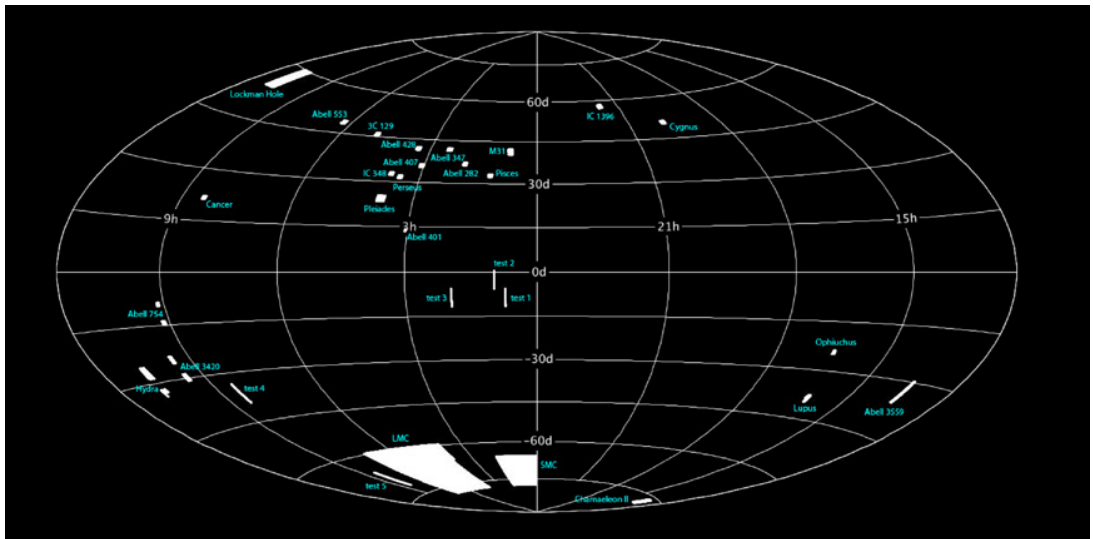

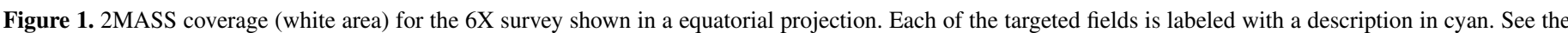
2MASS Explanatory Supplement (Cutri et al. 2003) for more information.

(A color version of this figure is available in the online journal.)

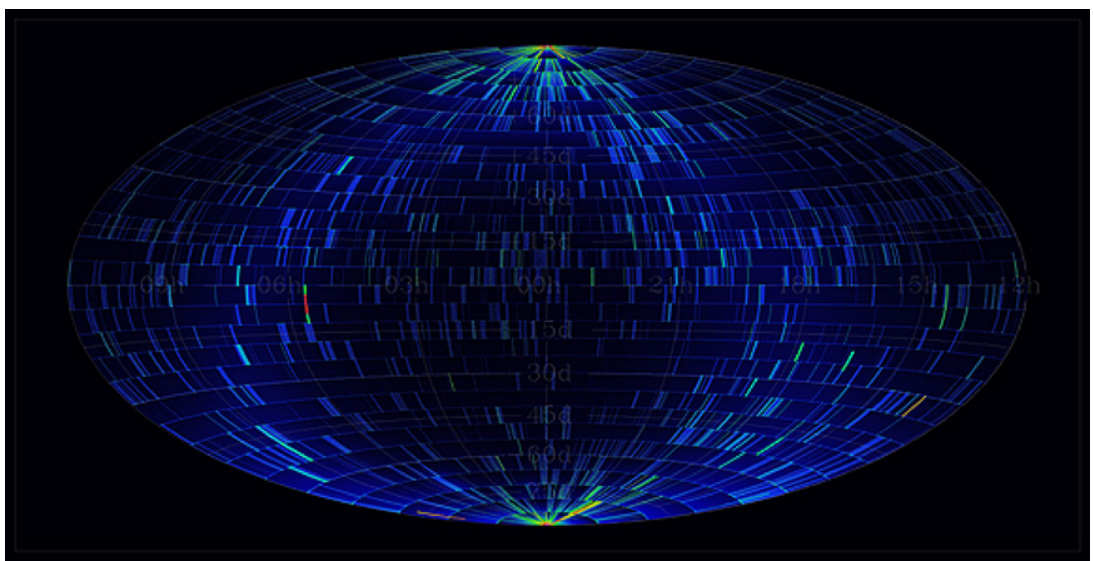

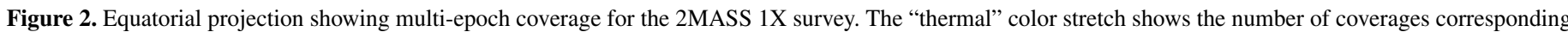

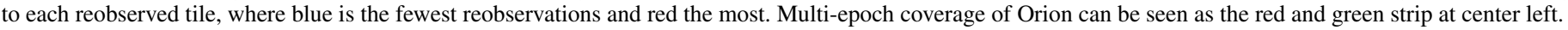
(A color version of this figure is available in the online journal.)

\subsection{The $1 X-1 X$ Survey}

\subsubsection{Pure Kinematic Selection (List 2)}

The second part of our survey used $1 \mathrm{X}$ data only. Although the 2MASS $1 \mathrm{X}$ survey was fundamentally a single-epoch coverage of the sky, approximately $30 \%$ of the sky was observed more than once in photometric conditions while the twin telescopes were in operation between 1997 and 2001. Figure 2 shows the sky coverage of the dual-epoch $1 \mathrm{X}$ data. Slightly more than half of this area is in the R.A. and decl. overlap regions between adjacent survey tiles. The remaining area is covered by tiles that were reobserved to improve minimum data quality, to provide additional survey validation, or to enable other scientific investigations. The 2MASS Survey Point and Extended Source Working Databases contain source information extracted during pipeline data reduction of all survey observations, and may therefore contain multiple, independent measurements of sources detected in the regions of the sky observed more than once. The 2MASS All-Sky Release Catalogs were constructed from the Working Databases but used only one measurement of multiply-detected sources to maintain survey uniformity.

To conduct this proper motion survey we used the 2MASS Survey Point Source Working Database (which is essentially a union of the 2MASS All-Sky Point Source Catalog and the 2MASS Survey Point Source Reject Table) and the 2MASS
Survey Merged Point Source Information Table. The Merged Point Source Information Table contains the averaged positional and photometric measurements for sources with more than one detection in the Point Source Working Database and was created by positionally autocorrelating the Point Source Survey Working Database to find "groups" of independent source extractions that fall within 1 ".5 of each other. The mean and rms position, the mean and rms brightness, and various detection statistics were computed for each associated group of detections and entered into the Merged Source Table. The merged source entries were also assigned warning flags if they could not be unambiguously separated from other nearby groups, if any of their constituents were resolved during pipeline data reduction, or if any of their constituents had measurements that were possibly contaminated by image artifacts.

We selected proper motion candidates in the Merged Point Source Information Table by using the IRSA/Gator search engine $^{16}$ to search for groups that satisfy the following criteria (conditions in parentheses after each description refer to the parameters listed in the Merged Point Source Table).

1. Are detected on two or more independent survey scans $($ sdet $\geqslant 2)$;

\footnotetext{
16 See http://irsa.ipac.caltech.edu.
} 
2. Have positional differences of at least $0^{\prime \prime} 4\left(0^{\circ} 000111\right)$ between the first and last detection epoch ${ }^{17}$ (sep_jdmax $\geqslant 0.000111$ ) and, by default (see above), positional differences of less than 1".5;

3. Have average $J$-band magnitudes brighter than $15.8 \mathrm{mag}$ $\left(j \_ \text {mavg } \leqslant 15.8\right)^{18}$;

4. Are not flagged as confused in the merging process $(\mathrm{gcnf}=0)$;

5. Contain no extended group members $\left(\mathrm{n} \_\right.$galcontam $=0$ ); and

6. Contain no group members with contaminated measurements $\left(\mathrm{ce} \_\right.$flg $\left.=0\right)$.

Candidate groups falling within $10^{\circ}$ of the Galactic Plane were further culled from the list, as source confusion there can severely limit the astrometric accuracy. The apparent proper motion of each candidate group was then computed by dividing the angular positional difference between the first and last epoch measurement by the time separating the observations. We imposed criteria of $\mu \geqslant 0.2 \mathrm{yr}^{-1}$ and epoch time differences $\geqslant 0.2 \mathrm{yr}$ for all of our proper motion candidates ${ }^{19}$. The constraint on epochal difference limits the search to a total area of $\sim 3440 \mathrm{deg}^{2}$. See Figure 6 of Section A2.1 in the 2MASS Explanatory Supplement (Cutri et al. 2003).

Finder charts for each candidate were constructed and examined by eye. These charts were comprised of five different images: 2MASS $J, H$, and $K_{s}$ bands; DSS $R, B$, or $V$ band; and XDSS $R$ band $^{20}$. This eliminated spurious sources that clearly showed no motion between the longer time baseline afforded by the DSS to 2MASS comparison. In the final analysis we identified 2301 proper motion candidates using these selections, $384(16.7 \%)$ of which are not listed in SIMBAD. Of these 2301 proper motion candidates, $225(9.8 \%)$ have no counterpart within a 3".0 radius in the USNO-B Catalog. A total of 37 (1.6\% of the original sample) are invisible by-eye on the DSS $B-, R$-, and $I$-band images and are thus 2MASS-only sources.

\subsubsection{Kinematic Selection with Imposed Color Criteria (List 3)}

As an adjunct to this second survey, we searched for proper motion candidates having fainter 2MASS $J$ magnitudes and/or smaller time and positional differences between the first and last 2MASS epochs; however, we restricted the search to portions of 2MASS color-color space where mid- to late- $\mathrm{L}$ and mid- to late-T dwarfs lie. The common constraints for these color-based surveys were the following.

1. Are not flagged as confused in the merging process $(\mathrm{gcnf}=0)$;

2. Contain no extended group members $\left(\mathrm{n} \_\right.$galcontam $=0$ ); and

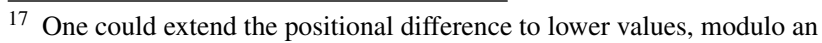
increase in the number of false motion sources because the positional difference would then be comparable to the single-epoch astrometric error.

${ }^{18}$ For objects with $0.000111 \leqslant s e p_{-} j d \max <0.000139 \mathrm{deg}$, the magnitude range $15.8 \mathrm{mag} \leqslant J \leqslant 16.0 \mathrm{mag}$ was searched only partially.

19 The maximum epoch difference for any of the 2MASS reobserved regions is about $3 \mathrm{yr}$. This means that only those sources with $\mu<7^{\prime \prime} .5 \mathrm{yr}^{-1}$ can be detected for cases with the smallest epoch difference, or $\mu<00^{\prime \prime} 5 \mathrm{yr}^{-1}$ for cases with the maximum difference. Because the epoch difference varies from spot to spot, it is not possible to place a fixed value on the maximum proper motion.

20 The DSS and XDSS images were pulled from the CADC service. See http://cadcwww.dao.nrc.ca/cadcbin/getdss. DSS refers to the First Generation Digitized Sky Survey and XDSS refers to the Second Generation Digitized Sky Survey.
}

3. Contain no group members with contaminated measurements $($ ce_flg $=0)$.

A search of L dwarf color space (specifically, j_mavg $h \_m a v g \geqslant 0.8 \mathrm{mag}$ and $h \_m a v g-k \_m a v g \geqslant 0.5 \mathrm{mag}$ ) included the additional constraints $0.000042 \leqslant s e p_{-} j d m a x<$ $0.000111 \mathrm{deg}$, sdet $\geqslant 2$, and epoch difference $\geqslant 0.1 \mathrm{yr}$. $\mathrm{T}$ dwarf color space had two searches performed on it, both using constraints $j \_$mavg $\leqslant 16.5 \mathrm{mag}$, epoch difference $\geqslant 0.1 \mathrm{yr}$, and total proper motion $\geqslant 0.2 \mathrm{yr}^{-1}$. The first of these searched color space with $j \_$mavg $-h \_$mavg $\leqslant 0.0$ mag and $h \_$mavg $k_{\_}$mavg $\leqslant 0.4 \mathrm{mag}$ and applied additional constraints of $s e p_{-} j d \max \geqslant 0.000028 \mathrm{deg}$ and $s d e t \geqslant 2$. The second of these searched $\mathrm{T}$ dwarf color space with $j \_$mavg $-k \_$mavg $\leqslant$ 0.5 mag and applied additional constraints of $s e p_{-} j d m a x \geqslant$ $0.000111 \mathrm{deg}$ and $s d e t \geqslant 3$.

Using these criteria, we identified 131 proper motion candidates. Of these, $82(62.6 \%)$ are not listed in SIMBAD and 79 (60.3\%) have no counterpart within a 3."0 radius in the USNO-B Catalog. A total of $62(47.3 \%)$ are invisible on the DSS $B-, R-$, and $I$-band images and hence are 2MASS-only sources.

\section{LIST OF PROPER MOTION CANDIDATES}

\subsection{Construction of the Table}

Table 1 gives composite information on all of these proper motion candidates. Columns 1-4 list general information on each target. Column 1 gives the name of the object as listed in the 2MASS All-Sky Survey Point Source Catalog; the name includes the sexagesimal $\mathbf{J} 2000$ position encoded as hhmmss[.]ss \pm ddmmss[.]s. Column 2 gives the list from which the proper motion candidate came: either List 1 (Section 2.1), List 2 (Section 2.2.1), or List 3 (Section 2.2.2), corresponding to the three searches above. For objects not visible on the $B$ or $R$-band plates of the Digitized Sky Surveys, Column 3 gives the shortest wavelength band where the object is seen $-I$ if it is visible in the DSS $I$-band plates or $J$ if it is seen only in 2MASS. Column 4 lists an asterisk for those objects we have followed up spectroscopically; follow-up observations for these are detailed in Table 2.

Columns 5-11 give 2MASS-specific data. Column 5 is the 2MASS-measured, absolute proper motion. For sources in List 1 this is computed by differencing the positional information from the 2MASS All-Sky Point Source Catalog (the earlier epoch) and the positional information for the same object in the 2MASS 6X Point Source Working Database (the later epoch) then dividing by the epoch difference; the error in the proper motion is the root sum square of the positional uncertainties (available for each source record in both catalogs) divided by the epoch difference. For sources in Lists 2 and 3 the calculations are the same except that the positional difference comes from the earliest and latest epochs contained in the 2MASS Point Source Working Database. Column 6 gives the position angle for the proper motion vector, calculated in the standard way as the number of degrees east of due north. To gauge the significance of the proper motion measurement, we give in Column 7 the astrometric displacement divided by the larger of the astrometric uncertainties in the two epochs. This value can be thought of as the number of standard deviations $(\sigma)$ that the positional difference lies away from zero motion. Columns 8-10 are the default $J, H$, and $K_{s}$ magnitudes and total photometric uncertainties; for List 1 sources these are $6 \mathrm{X}$ measurements taken from the 2MASS 6X Point Source Working Database/Catalog, and for List 2 and List 3 sources these 
Table 1

List of 2MASS Proper Motion Candidates

\begin{tabular}{|c|c|c|c|c|c|c|c|c|c|c|c|c|c|c|c|c|c|c|c|c|c|}
\hline $\begin{array}{l}\text { 2MASS Designation } \\
\text { J2000 Coords } \\
\text { (1) }\end{array}$ & $\begin{array}{l}\text { List } \\
\text { (2) }\end{array}$ & Band & $\begin{array}{c}\text { Spec. } \\
\text { (4) }\end{array}$ & $\begin{array}{c}\text { 2MASS PM } \\
\left(\operatorname{arcsec}^{-1} r^{-1}\right) \\
(5)\end{array}$ & $\begin{array}{c}\text { 2M Theta } \\
\text { (deg) } \\
\text { (6) }\end{array}$ & $\begin{array}{l}\text { Motion Sig. } \\
\text { (7) }\end{array}$ & $\begin{array}{c}J \\
(\mathrm{mag}) \\
(8)\end{array}$ & $\begin{array}{c}H \\
(\mathrm{mag}) \\
(9)\end{array}$ & $\begin{array}{c}K_{s} \\
(\mathrm{mag}) \\
(10)\end{array}$ & $\begin{array}{c}\mathrm{H}_{J} \\
(\mathrm{mag}) \\
(11) \\
\end{array}$ & $\begin{array}{c}\text { USNO-B PM } \\
\left(\begin{array}{c}\left.\operatorname{arcsec}_{\mathrm{yr}}^{-1}\right) \\
(12)\end{array}\right.\end{array}$ & $\begin{array}{l}\text { US Theta } \\
\text { (deg) } \\
(13)\end{array}$ & $\begin{array}{c}\text { B1 } \\
(\mathrm{mag}) \\
(14) \\
\end{array}$ & $\begin{array}{c}\mathrm{B} 2 \\
(\mathrm{mag}) \\
(15)\end{array}$ & $\begin{array}{c}\mathrm{R} 1 \\
(\mathrm{mag}) \\
(16)\end{array}$ & $\begin{array}{c}\mathrm{R} 2 \\
(\mathrm{mag}) \\
(17)\end{array}$ & $\begin{array}{c}\mathrm{I} \\
(\mathrm{mag}) \\
(18)\end{array}$ & $\begin{array}{c}\text { SIMBAD PM } \\
\left(\operatorname{arcsec}_{\mathrm{yr}^{-1}}\right) \\
(19)\end{array}$ & $\begin{array}{c}\begin{array}{c}\text { SI Theta } \\
\text { (deg) } \\
(20)\end{array} \\
\end{array}$ & $\begin{array}{c}\text { SIMBAD Name } \\
\text { (21) }\end{array}$ & $\begin{array}{c}\text { Spec. Type } \\
(22)\end{array}$ \\
\hline J00020936-7715065 & 1 & $\ldots$ & $\cdots$ & $0.26 \pm 0.12$ & 143 & 2.09 & $8.942 \pm 0.021$ & $8.462 \pm 0.026$ & $8.375 \pm 0.023$ & 11.00 & & & 11.54 & 11.07 & 10.19 & 10.14 & 9.76 & $0.21 \pm 0.01$ & 118 & NLTT 58838 & \\
\hline $0021076-4309560$ & 2 & $\ldots$ & $\ldots$ & $1.00 \pm 0.14$ & 137 & 6.74 & $12.597 \pm 0.026$ & $12.425 \pm 0.023$ & $12.445 \pm 0.024$ & 17.59 & $0.656 \pm 0.032$ & 181 & & 20.77 & & 13.04 & 12.40 & $1.02 \pm 0.14$ & 135 & LHS 1005 & DAs \\
\hline $0023356+4343145$ & 2 & $\ldots$ & $\ldots$ & $0.32 \pm 0.06$ & 81 & 4.90 & $12.629 \pm 0.021$ & $12.066 \pm 0.021$ & $11.954 \pm 0.018$ & 15.18 & $0.321 \pm 0.004$ & 78 & 16.10 & 15.65 & 14.28 & 14.31 & 13.79 & $0.35 \pm 0.02$ & 77 & G 171-37 & \\
\hline J00024468-2428426 & 2 & $\ldots$ & $\ldots$ & $0.41 \pm 0.07$ & 99 & 5.88 & $11.765 \pm 0.021$ & $11.204 \pm 0.023$ & $10.957 \pm 0.025$ & 14.84 & $0.404 \pm 0.003$ & 97 & 17.18 & 17.54 & 14.73 & 14.98 & 13.22 & $0.42 \pm 0.01$ & 98 & NLTT 10 & $\ldots$ \\
\hline J00024961-7636194 & 1 & $\ldots$ & $\ldots$ & $0.21 \pm 0.07$ & 267 & 3.20 & $9.884 \pm 0.023$ & $9.285 \pm 0.024$ & $9.118 \pm 0.023$ & 11.49 & $0.219 \pm$. & 237 & 13.14 & 12.76 & 11.98 & 11.94 & 11.60 & $0.22 \pm 0.01$ & 237 & NLTT 29 & $\ldots$ \\
\hline
\end{tabular}

(This table is available in its entirety in a machine-readable form in the online journal. A portion is shown here for guidance regarding its form and content.) 
Table 2

Spectroscopic Follow-up of Discoveries

\begin{tabular}{|c|c|c|c|c|c|c|c|}
\hline $\begin{array}{c}\text { 2MASS Designation } \\
\text { J2000 Coords } \\
(1)\end{array}$ & $\begin{array}{c}\text { Telescope } \\
\text { (2) }\end{array}$ & Instr. & $\begin{array}{l}\text { Obs. Date } \\
\text { (UT) } \\
(4)\end{array}$ & $\begin{array}{c}\text { Weather } \\
\text { (5) }\end{array}$ & $\begin{array}{l}\text { Int. } \\
(s) \\
(6)\end{array}$ & $\begin{array}{c}\text { Opt. Sp. Ty. } \\
\text { (7) }\end{array}$ & NIR Sp. Ty. \\
\hline 2MASS J00040288-6410358 & Magellan & LDSS3 & 2006 Nov 21 & Patchy clds & 2700 & $\mathrm{~L} 1 \gamma$ & $\ldots$ \\
\hline 2MASS J00054327+0836483 & Magellan & MagE & 2008 Nov 27 & Clear & 1500 & M4.5 & $\ldots$ \\
\hline \multirow[t]{2}{*}{ 2MASS J00054517+0723423 } & IRTF & SpeX & 2007 Oct 12 & Clear & 480 & $\ldots$ & sdM6 \\
\hline & Magellan & MagE & 2008 Nov 27 & Clear & 1500 & M4 & $\ldots$ \\
\hline \multirow[t]{2}{*}{ 2MASS J00150206+2959323 } & IRTF & SpeX & 2005 Oct 18 & Cirrus & 960 & $\ldots$ & L7.5 pec (blue) \\
\hline & Magellan & LDSS3 & 2008 Aug 13 & Clear & 1800 & L7 & $\ldots$ \\
\hline 2MASS J00163761+3448368 & IRTF & SpeX & 2005 Sep 09 & Cirrus & 960 & $\ldots$ & M8.5 \\
\hline 2MASS J00184613-6356122 & Magellan & LDSS3 & 2006 Nov 21 & Patchy clds & 900 & $\mathrm{~L} 2$ & $\ldots$ \\
\hline 2MASS J00252923-8910199 & Magellan & LDSS3 & 2006 Aug 28 & Clear? & 1800 & M7 & $\ldots$ \\
\hline 2MASS J00451330+1228538 & IRTF & SpeX & 2006 Aug 28 & lt. cirrus & 960 & $\ldots$ & M8.5 \\
\hline 2MASS J00524886+1712434 & IRTF & SpeX & 2008 Oct 12 & 1t. cirrus & 960 & $\ldots$ & esdM5 \\
\hline \multirow[t]{2}{*}{ 2MASS J00554279+1301043 } & IRTF & SpeX & 2006 Dec 20 & Clear & 960 & $\ldots$ & $\mathrm{d} / \mathrm{sdM} 7$ \\
\hline & Subaru & FOCAS & 2007 Aug 21 & Clear & 1200 & M6 & $\ldots$ \\
\hline \multirow[t]{2}{*}{ 2MASS J01153252-7519079f } & Gemini-South & GMOS & 2004 Dec 08 & Clear & 2400 & M7 & $\ldots$ \\
\hline & Magellan & LDSS3 & 2005 Dec 02 & Clear & 2700 & M7 & $\ldots$ \\
\hline 2MASS J01170586+1752568 & IRTF & SpeX & 2005 Sep 08 & Clear & 960 & $\ldots$ & M8 \\
\hline 2MASS J01251802-4545310 & IRTF & SpeX & 2006 Sep 01 & Clear & 400 & $\ldots$ & WD \\
\hline 2MASS J01330461-1355271 & IRTF & SpeX & 2005 Oct 20 & Clear & 960 & $\ldots$ & M8 \\
\hline 2MASS J01340012-7239330 & Gemini-South & GMOS & 2004 Sep 13 & Clear & 2400 & M6 & $\ldots$ \\
\hline \multirow[t]{2}{*}{ 2MASS J01340281+0508125 } & Keck-II & NIRSPEC & 2005 Jul 18 & Clear & 1200 & $\ldots$ & $\mathrm{L} 2^{\mathrm{a}}$ \\
\hline & IRTF & SpeX & 2006 Dec 08 & Clear & 1440 & $\ldots$ & L1 \\
\hline 2MASS J01405263+0453302 & IRTF & SpeX & 2006 Aug 17 & Clear & 720 & $\ldots$ & M5.5 \\
\hline 2MASS J01414839-1601196 & IRTF & SpeX & 2005 Oct 18 & Cirrus & 960 & $\ldots$ & L7: \\
\hline 2MASS J01452055-7322292 & Gemini-South & GMOS & 2004 Dec 08 & Clear & 1200 & M6.5 & $\ldots$ \\
\hline 2MASS J01472702+4731142 & IRTF & SpeX & 2006 Aug 28 & lt. cirrus & 960 & $\ldots$ & L1.5 \\
\hline 2MASS J01481478+1202447A & IRTF & SpeX & 2007 Oct 12 & Clear & 80 & $\ldots$ & M3 \\
\hline 2MASS J01481478+1202447B & IRTF & SpeX & 2007 Oct 12 & Clear & 80 & $\ldots$ & WD \\
\hline 2MASS J01574939-1146113 & IRTF & SpeX & 2005 Sep 09 & Cirrus & 840 & $\ldots$ & M9: \\
\hline 2MASS J02304442-3027275 & IRTF & SpeX & 2006 Sep 01 & Clear & 880 & $\cdots$ & $\mathrm{L} 1$ \\
\hline 2MASS J02330145-6403420 & Magellan & LDSS3 & 2005 Oct 31 & Clear & 1200 & $\mathrm{~L} 2$ & $\ldots$ \\
\hline 2MASS J02340093-6442068 & Magellan & LDSS3 & 2005 Dec 04 & Clear & 1800 & $\mathrm{~L} 0 \gamma$ & $\ldots$ \\
\hline 2MASS J02465552-8247010 & Magellan & LDSS3 & 2005 Dec 04 & Clear & 1500 & M8 & $\ldots$ \\
\hline \multirow[t]{2}{*}{ 2MASS J02481204+2445141 } & IRTF & SpeX & 2006 Aug 18 & Clear & 480 & $\ldots$ & M8: \\
\hline & IRTF & SpeX & 2006 Dec 09 & lt. cirrus & 960 & $\ldots$ & M8 \\
\hline 2MASS J03001631+2130205 & IRTF & SpeX & 2006 Aug 28 & 1t. cirrus & 840 & $\ldots$ & L6 pec (sl. blue) \\
\hline 2MASS J03032042-7312300 & Magellan & LDSS3 & 2006 Nov 21 & Patchy clds & 1200 & $\mathrm{~L} 2 \gamma$ & $\ldots$ \\
\hline 2MASS J03073402-1736375 & Magellan & LDSS3 & 2005 Nov 01 & Clear & 3600 & usdM2 & $\ldots$ \\
\hline 2MASS J03253013-7644247 & Magellan & MagE & 2009 Jan 08 & Clear & 2400 & M6 & $\ldots$ \\
\hline 2MASS J03280716+3022432 & IRTF & SpeX & 2006 Sep 02 & Clear & 960 & $\ldots$ & (bkg) \\
\hline 2MASS J03305571+3146272 & IRTF & SpeX & 2003 Sep 18 & Thin cirrus & 480 & $\cdots$ & M8 \\
\hline 2MASS J03382862+0001296 & IRTF & SpeX & 2006 Sep 02 & Clear & 800 & $\ldots$ & M7.5 \\
\hline 2MASS J03422594+3148496 & IRTF & SpeX & 2005 Oct 18 & Cirrus & 960 & $\ldots$ & (bkg) \\
\hline 2MASS J03431581+3210455 & IRTF & SpeX & 2006 Sep 02 & Clear & 960 & $\ldots$ & (giant) \\
\hline 2MASS J03440399+2730504 & Keck-I & LRIS & 2009 Oct 12 & Clear & 1200 & sdM3.5 & $\ldots$ \\
\hline 2MASS J03444306+3137338 & IRTF & SpeX & 2005 Oct 18 & Cirrus & 480 & $\ldots$ & (M giant) \\
\hline 2MASS J03542527+3724388 & IRTF & SpeX & 2006 Sep 02 & Clear & 960 & $\ldots$ & (bkg) \\
\hline 2MASS J03544410-4311383 & Magellan & LDSS3 & 2005 Dec 02 & Clear & 1500 & M6 & $\ldots$ \\
\hline 2MASS J03551017-0333210 & Keck-I & LRIS & 2009 Oct 12 & Clear & 600 & M8.5 & $\ldots$ \\
\hline 2MASS J04024153-4037476 & IRTF & SpeX & 2007 Nov 10 & Clear & 360 & $\ldots$ & WD \\
\hline \multirow[t]{2}{*}{ 2MASS J04062677-3812102 } & IRTF & SpeX & 2006 Dec 08 & Clear & 960 & $\ldots$ & L1 pec (low-g) \\
\hline & Keck-I & LRIS & 2009 Oct $11 / 12$ & Clear & 8400 & $\mathrm{~L} 0 \gamma$ & $\ldots$ \\
\hline 2MASS J04150602+8230557 & Gemini-North & GMOS & 2006 Dec 23 & $\ldots$ & 1800 & L3 & $\ldots$ \\
\hline 2MASS J04291099-6649434 & Gemini-South & GMOS & 2004 Dec 07 & Thin cirrus & 2400 & M9.5 & $\ldots$ \\
\hline \multirow[t]{3}{*}{ 2MASS J04382218+2553503 } & IRTF & SpeX & 2005 Dec 31 & lt. cirrus & & $\ldots$ & (bkg) \\
\hline & IRTF & SpeX & 2006 Aug 28 & lt. cirrus & 480 & $\ldots$ & (bkg) \\
\hline & IRTF & SpeX & 2006 Dec 09 & lt. cirrus & 960 & $\ldots$ & (bkg) \\
\hline \multirow[t]{2}{*}{ 2MASS J04470652-1946392 } & IRTF & SpeX & 2004 Sep 08 & $\cdots$ & & $\cdots$ & sdM7: \\
\hline & IRTF & SpeX & 2006 Dec 23 & $\ldots$ & & $\ldots$ & sdM7.5 \\
\hline
\end{tabular}


Table 2

(Continued)

\begin{tabular}{|c|c|c|c|c|c|c|c|}
\hline $\begin{array}{l}\text { 2MASS Designation } \\
\text { J2000 Coords } \\
(1)\end{array}$ & $\begin{array}{c}\text { Telescope } \\
\text { (2) }\end{array}$ & $\begin{array}{l}\text { Instr. } \\
\text { (3) }\end{array}$ & $\begin{array}{l}\text { Obs. Date } \\
\text { (UT) } \\
(4)\end{array}$ & $\begin{array}{c}\text { Weather } \\
\text { (5) }\end{array}$ & $\begin{array}{l}\text { Int. } \\
(\mathrm{s}) \\
(6)\end{array}$ & $\begin{array}{l}\text { Opt. Sp. Ty. } \\
\text { (7) }\end{array}$ & $\begin{array}{l}\text { NIR Sp. Ty. } \\
\text { (8) }\end{array}$ \\
\hline 2MASS J04484476-6401100 & Gemini-South & GMOS & 2004 Dec 08 & clear & 2400 & M7.5 & $\theta$ \\
\hline 2MASS J05253876-7426008 & Gemini-South & GMOS & 2004 Dec 08 & clear & 3600 & $\mathrm{~L} 2$ & \\
\hline 2MASS J05314149+6856293 & IRTF & SpeX & 2006 Dec 09 & 1t. cirrus & 960 & $\ldots$ & M9 \\
\hline \multirow{2}{*}{ 2MASS J05341594-0631397 } & IRTF & SpeX & 2006 Dec 08 & clear & 720 & $\ldots$ & M8 pec (low-g) \\
\hline & Keck-I & LRIS & 2009 Oct 11 & clear & 1200 & M8 $\gamma$ & $\ldots$ \\
\hline 2MASS J05363713-0328492 & IRTF & SpeX & 2006 Sep 30 & $\ldots$ & 480 & $\ldots$ & M8 \\
\hline 2MASS J05363776+1000232 & IRTF & SpeX & 2006 Dec 09 & 1t. cirrus & 960 & $\ldots$ & (giant?) \\
\hline 2MASS J05381018-0554261 & IRTF & SpeX & 2006 Dec 08 & clear & 960 & $\ldots$ & (bkg) \\
\hline 2MASS J05460407-0003228 & IRTF & SpeX & 2006 Dec 08 & clear & 720 & $\ldots$ & (bkg) \\
\hline 2MASS J05484335-7151165 & Magellan & LDSS3 & 2005 Oct 31 & clear & 1800 & (bkg) & $\ldots$ \\
\hline 2MASS J05593843-6939230 & Gemini-South & GMOS & 2004 Dec 08 & clear & 1200 & M7 & $\ldots$ \\
\hline 2MASS J06122818-8556480 & Magellan & LDSS3 & 2005 Nov 01 & clear? & 1800 & M7 & $\ldots$ \\
\hline \multirow[t]{2}{*}{ 2MASS J06211484+6558164 } & IRTF & SpeX & 2006 Dec 21 & clear & 320 & $\ldots$ & $<$ esdM5 \\
\hline & Keck-I & LRIS & 2009 Oct 12 & clear & 360 & esdM0 & $\ldots$ \\
\hline 2MASS J06272155+7354319 & Gemini-North & GMOS & 2006 Nov 18 & $\ldots$ & 1800 & M7 & $\ldots$ \\
\hline 2MASS J06414609-7909036 & Magellan & LDSS3 & 2005 Dec 03 & clear & 2700 & M7 & $\ldots$ \\
\hline 2MASS J06423542-7506067 & Gemini-South & GMOS & 2004 Dec 09 & clear & 2400 & M6.5 & $\ldots$ \\
\hline \multirow[t]{3}{*}{ 2MASS J06453153-6646120 } & Gemini-South & GMOS & 2004 Dec 14 & clear & 3600 & sdL8 & $\ldots$ \\
\hline & SOAR & OSIRIS & 2007 Mar 13 & clear & 1080 & $\ldots$ & sdL8 \\
\hline & Magellan & LDSS3 & 2008 Feb 23 & clear & 1800 & sdL8 & $\ldots$ \\
\hline 2MASS J07340502+5810484 & IRTF & SpeX & 2007 Nov 11 & clear & 1800 & $\ldots$ & $<\mathrm{M} 3$ \\
\hline 2MASS J07415784+0531568 & IRTF & SpeX & 2006 Apr 08 & cirrus/clds & 720 & $\ldots$ & $\mathrm{L} 1.5$ \\
\hline 2MASS J07552301-8104368 & Magellan & LDSS3 & 2006 May 06 & clear & 1800 & M6 & $\ldots$ \\
\hline \multirow[t]{3}{*}{ 2MASS J09171104-1650010 } & IRTF & SpeX & 2006 Apr 09 & $\ldots$ & 1440 & $\ldots$ & M7 \\
\hline & IRTF & SpeX & 2008 Jan 25 & cirrus & 720 & $\ldots$ & M7 \\
\hline & Magellan & LDSS3 & 2008 Feb 24 & clear & 1500 & M7 & $\ldots$ \\
\hline 2MASS J09243114+2143536 & IRTF & SpeX & 2006 Jun 01 & Clear & 960 & $\ldots$ & M7.5 \\
\hline 2MASS J09384022-2748184 & IRTF & SpeX & 2006 Apr 10 & $\ldots$ & $120(?)$ & $\ldots$ & M7.5 \\
\hline 2MASS J09395909-3817217 & IRTF & SpeX & 2006 Apr 11 & $\ldots$ & 300 & $\ldots$ & M7: \\
\hline 2MASS J10042715-4221314 & Magellan & LDSS3 & 2006 May 06 & Clear & 900 & M7 & $\ldots$ \\
\hline 2MASS J10315064+3349595 & IRTF & SpeX & 2006 Jun 01 & Clear & 960 & $\ldots$ & $\mathrm{L} 2$ \\
\hline 2MASS J10334372-3337056 & IRTF & SpeX & 2008 Jan 13 & $\ldots$ & 1200 & $\ldots$ & M5.5 \\
\hline 2MASS J10363150-2714487 & Keck-I & LRIS & 2003 Dec 22 & Clear & 300 & M7 & $\ldots$ \\
\hline 2MASS J10433508+1213149 & IRTF & SpeX & 2006 May 31 & Clear & 960 & $\ldots$ & L9 \\
\hline 2MASS J10452428-2254481 & Magellan & LDSS3 & 2006 May 07 & Clear & 1200 & M8 & $\ldots$ \\
\hline 2MASS J10454932+1254541 & IRTF & SpeX & 2006 Jan 04 & $\ldots$ & 1200 & $\ldots$ & M8 \\
\hline \multirow[t]{2}{*}{ 2MASS J10461875+4441149 } & Keck-II & NIRSPEC & 2005 Dec 09 & Clear & 1200 & $\ldots$ & $\mathrm{L} 4^{\mathrm{a}}$ \\
\hline & IRTF & SpeX & 2006 Dec 08 & Clear & 960 & $\ldots$ & L5 pec (sl. blue) \\
\hline 2MASS J10462067+2354307 & IRTF & SpeX & 2008 Jan 13 & $\ldots$ & 960 & $\ldots$ & sdM6 \\
\hline 2MASS J11001126-2718386 & Magellan & LDSS3 & 2006 May 07 & Clear & 750 & L1 & $\ldots$ \\
\hline \multirow[t]{2}{*}{ 2MASS J11061191+2754215 } & Spitzer & IRS & 2005.958 & $(\mathrm{~N} / \mathrm{A})$ & 3904 & $\ldots$ & $\ldots$ \\
\hline & IRTF & SpeX & 2006 Apr 08 & Cirrus/clds & 480 & & $\mathrm{~T} 2.5$ \\
\hline 2MASS J11151545-1657098 & Magellan & LDSS3 & 2006 May 08 & 1t. cirrus & 1800 & M7.5 & $\ldots$ \\
\hline \multirow[t]{3}{*}{ 2MASS J11181292-0856106 } & Magellan & LDSS3 & 2006 May 08 & lt. cirrus & 1800 & L6 & $\ldots$ \\
\hline & IRTF & SpeX & 2008 Jan 14 & $\ldots$ & 900 & $\ldots$ & L5: pec (blue) \\
\hline & IRTF & SpeX & 2008 Apr 28 & Hazy & 960 & $\ldots$ & L6 pec (blue) \\
\hline 2MASS J11304030+1206306 & IRTF & SpeX & 2008 Jan 13 & $\ldots$ & 960 & $\ldots$ & M6.5 \\
\hline 2MASS J11391441-4321106 & Magellan & LDSS3 & 2006 May 06 & Clear & 2400 & $\mathrm{~L} 2.5$ & $\ldots$ \\
\hline \multirow[t]{3}{*}{ 2MASS J11582077+0435014 } & Keck-II & NIRSPEC & 2005 Dec 09 & Clear & 1200 & $\ldots$ & $\operatorname{sdL} 7^{\mathrm{a}}$ \\
\hline & IRTF & SpeX & 2006 Apr 08 & Cirrus/clds & 1080 & . & sdL7 \\
\hline & Keck-I & LRIS & 2009 Feb 17 & Clear & 2400 & sdL7 & $\ldots$ \\
\hline 2MASS J12154938-0036387 & IRTF & SpeX & 2006 Apr 10 & $\ldots$ & 750 & $\ldots$ & M7.5 \\
\hline 2MASS J12171942-4830012 & Magellan & LDSS3 & 2006 May 06 & Clear & 3000 & L1 & $\ldots$ \\
\hline 2MASS J12304562+2827583 & IRTF & SpeX & $2007 \mathrm{Jul} 03$ & Clear & 480 & $\ldots$ & L4: \\
\hline 2MASS J12341814+0008359 & IRTF & SpeX & 2006 Apr 10 & $\ldots$ & 360 & $\ldots$ & M7 \\
\hline 2MASS J12414645-2238178 & IRTF & SpeX & 2006 Jun 01 & Clear & 480 & $\ldots$ & M8.5 \\
\hline 2MASS J12425052+2357231 & IRTF & SpeX & 2006 Apr 09 & $\ldots$ & 1080 & $\ldots$ & M9 \\
\hline 2MASS J12471472-0525130 & IRTF & SpeX & 2006 Apr 10 & $\ldots$ & 630 & $\ldots$ & M4.5 \\
\hline 2MASS J12474944-1117551 & IRTF & SpeX & 2007 Jul 03 & Clear & 960 & $\ldots$ & L0: \\
\hline 2MASS J12490872+4157286 & IRTF & SpeX & 2007 Mar 18 & Clear & 960 & $\ldots$ & M9 \\
\hline \multirow{2}{*}{ 2MASS J13023811+5650212 } & IRTF & SpeX & $2007 \mathrm{Jul} 04$ & 1t. cirrus & 960 & $\ldots$ & L3: pec (sl. blue) \\
\hline & Keck-I & LRIS & 2009 Feb 17 & Clear & 2400 & L2 & $\ldots$ \\
\hline 2MASS J13032137+2351110 & IRTF & SpeX & 2006 Apr 09 & $\ldots$ & 80 & $\ldots$ & $<\mathrm{M} 3$ \\
\hline 2MASS J13080147+3553169 & IRTF & SpeX & 2007 Mar 18 & Clear & 960 & $\cdots$ & M8 \\
\hline
\end{tabular}


Table 2

(Continued)

\begin{tabular}{|c|c|c|c|c|c|c|c|}
\hline $\begin{array}{l}\text { 2MASS Designation } \\
\text { J2000 Coords } \\
\text { (1) }\end{array}$ & $\begin{array}{c}\text { Telescope } \\
\text { (2) }\end{array}$ & $\begin{array}{l}\text { Instr. } \\
\text { (3) }\end{array}$ & $\begin{array}{l}\text { Obs. Date } \\
\text { (UT) } \\
(4)\end{array}$ & $\begin{array}{c}\text { Weather } \\
\text { (5) }\end{array}$ & $\begin{array}{l}\text { Int. } \\
(s) \\
(6) \\
\end{array}$ & $\begin{array}{l}\text { Opt. Sp. Ty. } \\
\text { (7) }\end{array}$ & $\begin{array}{l}\text { NIR Sp. Ty. } \\
\text { (8) }\end{array}$ \\
\hline 2MASS J13133360-0536110 & Magellan & LDSS3 & 2006 May 07 & Clear & 900 & M7 & $\ldots$ \\
\hline 2MASS J13141212-0324550 & Magellan & LDSS3 & 2006 May 07 & Clear & 1650 & M6 & $\ldots$ \\
\hline 2MASS J13151802+1103169 & Magellan & LDSS3 & 2006 May 08 & lt. cirrus & 1200 & M8 & $\ldots$ \\
\hline 2MASS J13184794+1736117 & IRTF & SpeX & 2007 Mar 18 & Clear & 960 & $\ldots$ & L5.5 \\
\hline 2MASS J13243553+6358281 & IRTF & SpeX & 2006 Apr 11 & $\ldots$ & 600 & $\ldots$ & $\mathrm{T} 2.5 \mathrm{pec}$ \\
\hline \multirow{2}{*}{ 2MASS J13313310+3407583 } & IRTF & SpeX & 2007 Jul 04 & lt. cirrus & 720 & $\ldots$ & L1 pec (red) \\
\hline & Keck-I & LRIS & 2009 Feb 17 & Clear & 1200 & L0 & $\ldots$ \\
\hline 2MASS J13341216-8226232 & Magellan & LDSS3 & 2006 May 07 & Clear & 1050 & L0 & $\ldots$ \\
\hline 2MASS J13342806+5258199 & IRTF & SpeX & 2007 Mar 18 & Clear & 480 & $\ldots$ & M7.5 \\
\hline 2MASS J13544271+2150309 & IRTF & SpeX & 2006 Jun 01 & Clear & 480 & $\ldots$ & M6 \\
\hline \multirow[t]{2}{*}{ 2MASS J14044948-3159330 } & Magellan & LDSS3 & 2006 May 08 & lt. cirrus & 3000 & ear- $\mathrm{T}$ & $\ldots$ \\
\hline & IRTF & SpeX & 2006 Jun 01 & Clear & 1440 & $\ldots$ & $\mathrm{T} 3$ \\
\hline 2MASS J14075592-3033593 & Magellan & LDSS3 & 2006 May 08 & lt. cirrus & 1350 & L1 & $\ldots$ \\
\hline 2MASS J14113696+2112471 & IRTF & SpeX & 2006 Apr 10 & $\ldots$ & 300 & $\ldots$ & M3.5 \\
\hline 2MASS J14182962-3538060 & IRTF & SpeX & 2006 Apr 11 & $\ldots$ & 900 & $\ldots$ & L1.5 \\
\hline 2MASS J14185890+6000194 & IRTF & SpeX & 2006 Aug 28 & 1t. cirrus & 960 & $\ldots$ & M9 \\
\hline 2MASS J14192618+5919047 & IRTF & SpeX & 2007 Jul 04 & lt. cirrus & 1200 & $\ldots$ & L1: \\
\hline 2MASS J14270666+4808164 & IRTF & SpeX & 2008 Jan 11 & $\ldots$ & 360 & $\ldots$ & $\leqslant$ sdM5 \\
\hline 2MASS J14344976-3121454 & Magellan & LDSS3 & 2006 May 08 & lt. cirrus & 1050 & M9.5 & $\ldots$ \\
\hline 2MASS J14370929-3512430 & IRTF & SpeX & 2007 Jul 03 & Clear & 480 & $\ldots$ & M8.5 \\
\hline \multirow[t]{2}{*}{ 2MASS J14403186-1303263 } & IRTF & SpeX & 2006 May 31 & Clear & 960 & $\ldots$ & L1 pec (sl. blue) \\
\hline & Magellan & LDSS3 & 2008 Aug 12 & Clear & 1200 & L1 & $\ldots$ \\
\hline 2MASS J14532582+1420410 & Keck-II & NIRSPEC & $2005 \mathrm{Jul} 19$ & Clear & 1200 & $\ldots$ & $\mathrm{L} 1^{\mathrm{a}}$ \\
\hline 2MASS J14594626+0004427 & Keck-II & NIRSPEC & $2005 \mathrm{Jul} 19$ & Clear & 1200 & $\ldots$ & $<\mathrm{M}^{\mathrm{a}}$ \\
\hline 2MASS J15111466+0607431 & IRTF & SpeX & $2007 \mathrm{Jul} 28$ & Clear & 960 & $\ldots$ & $\mathrm{T} 2 \mathrm{pec}$ \\
\hline 2MASS J15141384+1201451 & IRTF & SpeX & 2006 Apr 10 & $\ldots$ & 540 & $\ldots$ & M8 \\
\hline 2MASS J15200224-4422419A & IRTF & SpeX & 2006 Apr 08 & Cirrus/clds & 540 & $\ldots$ & $\mathrm{L} 1.5^{\mathrm{c}}$ \\
\hline 2MASS J15200224-4422419B & IRTF & SpeX & 2006 Apr 08 & Cirrus/clds & 720 & $\ldots$ & $\mathrm{L} 4.5^{\mathrm{c}}$ \\
\hline 2MASS J15201746-1755307 & IRTF & SpeX & 2006 Apr 10 & $\ldots$ & 360 & $\ldots$ & M8 \\
\hline 2MASS J15293306+6730215 & IRTF & SpeX & $2007 \mathrm{Jul} 28$ & Clear & 480 & $\ldots$ & L0: \\
\hline \multirow[t]{3}{*}{ 2MASS J15575011-2952431 } & IRTF & SpeX & 2006 Jun 01 & Clear & 960 & $\ldots$ & M9 pec (low-g) \\
\hline & IRTF & SpeX & 2008 Jul 29 & Cirrus & 1280 & $\ldots$ & M9 pec (low-g) \\
\hline & Magellan & LDSS3 & 2008 Aug 12 & Clear & 1800 & M9 $\delta$ & $\ldots$ \\
\hline 2MASS J16002647-2456424 & IRTF & SpeX & 2006 Aug 28 & lt. cirrus & 960 & $\ldots$ & M7.5 pec \\
\hline 2MASS J16130315+6502051 & IRTF & SpeX & 2008 Jul 30 & Clear & 900 & $\ldots$ & sdM6 \\
\hline 2MASS J16293044-2912524 & Keck-II & NIRSPEC & $2005 \mathrm{Jul} 18$ & Clear & 1200 & $\ldots$ & $(\mathrm{bkg})^{\mathrm{a}}$ \\
\hline 2MASS J16382073+1327354 & IRTF & SpeX & 2006 Sep 02 & Clear & 960 & $\ldots$ & $\mathrm{L} 2$ \\
\hline 2MASS J16390818+2839015 & IRTF & SpeX & 2006 Sep 02 & Clear & 960 & $\ldots$ & M8 \\
\hline \multirow[t]{2}{*}{ 2MASS J16423481-2355027 } & IRTF & SpeX & 2006 Sep 12 & lt. cirrus & 960 & $\ldots$ & (bkg) \\
\hline & IRTF & SpeX & 2007 Jul 28 & Clear & 480 & $\ldots$ & (bkg) \\
\hline 2MASS J17033593+2119071 & IRTF & SpeX & 2006 May 31 & Clear & 960 & $\ldots$ & M9 \\
\hline 2MASS J17220990-1158127A & IRTF & SpeX & 2006 Sep 12 & lt. cirrus & 480 & $\ldots$ & M5.5: \\
\hline 2MASS J17220990-1158127B & IRTF & SpeX & 2006 Sep 12 & 1t. cirrus & 480 & $\ldots$ & M7: \\
\hline 2MASS J17264629-1158036 & IRTF & SpeX & 2006 Sep 12 & 1t. cirrus & 960 & $\cdots$ & M5.5: \\
\hline 2MASS J17320014+2656228 & IRTF & SpeX & 2006 Jun 01 & Clear & 960 & $\ldots$ & L1 \\
\hline 2MASS J17343053-1151388 & IRTF & SpeX & 2005 Sep 08 & Clear & 480 & $\ldots$ & M8.5 \\
\hline 2MASS J17392515+2454421 & IRTF & SpeX & 2005 Oct 20 & Clear & 960 & $\ldots$ & $\mathrm{L} 4$ \\
\hline \multirow[t]{4}{*}{ 2MASS J17561080+2815238 } & IRTF & SpeX & 2005 Oct 20 & Clear & 840 & $\ldots$ & L1 pec (blue) \\
\hline & Gemini-North & GMOS & 2006 Jul 29 & $\ldots$ & 1800 & $\operatorname{sdL} 1$ & $\ldots$ \\
\hline & Keck-II & NIRSPEC & 2006 Aug 04 & Clear & 1200 & $\ldots$ & L1 pec \\
\hline & Subaru & FOCAS & 2007 Aug 21 & Clear & 1800 & sdL1 & $\ldots$ \\
\hline 2MASS J18131803+5101246 & IRTF & SpeX & 2006 May 31 & Clear & 960 & $\ldots$ & L5 \\
\hline \multirow[t]{4}{*}{ 2MASS J18212815+1414010 } & Keck-II & NIRSPEC & 2005 Jul 18 & Clear & 1200 & $\ldots$ & $\mathrm{L}^{\mathrm{a}}$ \\
\hline & IRTF & SpeX & 2005 Aug 10 & Clear & 480 & $\ldots$ & L5 pec (red) \\
\hline & Spitzer & IRS & 2006.304 & $(\mathrm{~N} / \mathrm{A})$ & 3904 & $\ldots$ & $\ldots$ \\
\hline & Subaru & FOCAS & 2007 Aug 20 & Clear & 2400 & L4.5 & $\ldots$ \\
\hline 2MASS J18282794+1453337 & IRTF & SpeX & 2005 Oct 20 & Clear & 960 & $\ldots$ & M8.5 \\
\hline \multirow[t]{4}{*}{ 2MASS J18284076+1229207 } & IRTF & SpeX & 2005 Sep 08 & Clear & 480 & $\ldots$ & M7.5 pec \\
\hline & Spitzer & IRS & 2006.302 & $(\mathrm{~N} / \mathrm{A})$ & 2562 & $\ldots$ & $\ldots$ \\
\hline & Gemini-North & GMOS & 2006 Jun 29 & $\ldots$ & 1200 & M8 & $\ldots$ \\
\hline & IRTF & SpeX & 2008 Aug 28 & $\ldots$ & 840 & $\ldots$ & M7.5 pec \\
\hline \multirow[t]{3}{*}{ 2MASS J18355309-3217129 } & Keck-II & NIRSPEC & 2005 Jul 19 & Clear & 1200 & $\ldots$ & M7 pec ${ }^{\mathrm{a}}$ \\
\hline & IRTF & SpeX & 2005 Aug 10 & poor(?) & 900 & $\ldots$ & $\mathrm{d} / \mathrm{sdM} 7$ \\
\hline & Magellan & LDSS3 & 2008 Oct 05 & Clear & 1200 & M6.5 & $\ldots$ \\
\hline
\end{tabular}


Table 2

(Continued)

\begin{tabular}{|c|c|c|c|c|c|c|c|}
\hline $\begin{array}{c}\text { 2MASS Designation } \\
\text { J2000 Coords } \\
\text { (1) }\end{array}$ & $\begin{array}{c}\text { Telescope } \\
\text { (2) }\end{array}$ & $\begin{array}{l}\text { Instr. } \\
\text { (3) }\end{array}$ & $\begin{array}{l}\text { Obs. Date } \\
\text { (UT) } \\
(4)\end{array}$ & $\begin{array}{c}\text { Weather } \\
\text { (5) }\end{array}$ & $\begin{array}{l}\text { Int. } \\
(s) \\
(6)\end{array}$ & $\begin{array}{l}\text { Opt. Sp. Ty. } \\
\text { (7) }\end{array}$ & $\begin{array}{l}\text { NIR Sp. Ty. } \\
\text { (8) }\end{array}$ \\
\hline 2MASS J18505160-5019113 & Magellan & LDSS3 & 2006 May 08 & 1t. cirrus & 1500 & L4 & \\
\hline 2MASS J19163888-3700026 & IRTF & SpeX & 2006 May 31 & Clear & 960 & $\ldots$ & L1 \\
\hline 2 MASS J19240858+5506524 ${ }^{\mathrm{d}}$ & Gemini-North & GMOS & 2006 Jul 29 & $\ldots$ & 360 & M7.5 & $\ldots$ \\
\hline 2MASS J19415458+6826021 & IRTF & SpeX & 2006 Jun 01 & Clear & 960 & $\ldots$ & L2 \\
\hline 2MASS J19475198-3438572 & IRTF & SpeX & 2005 Oct 19 & cirrus & 960 & $\ldots$ & M7.5 \\
\hline \multirow[t]{2}{*}{ 2MASS J19495702+6222440 } & IRTF & SpeX & 2006 Aug 28 & 1t. cirrus & 720 & $\ldots$ & L2 pec (sl. blue) \\
\hline & Keck-I & LRIS & 2009 Oct 12 & Clear & 4800 & L2 pec (blue) & \\
\hline 2MASS J20033545+1158552 & IRTF & SpeX & 2006 Jun 01 & Clear & 840 & $\ldots$ & M5.5 \\
\hline 2MASS J20111253+7153122 & Gemini-North & GMOS & 2006 Sep 19 & $\ldots$ & 1800 & $\mathrm{~L} 2$ & $\ldots$ \\
\hline 2MASS J20283175+6925116 & IRTF & SpeX & 2006 Sep 02 & Clear & 400 & $\ldots$ & M4.5 \\
\hline 2MASS J20342948+6727398 & IRTF & SpeX & 2005 Oct 20 & Clear & 600 & $\ldots$ & (bkg) \\
\hline 2MASS J20553767+7443343 & Gemini-North & GMOS & 2006 Aug 30 & $\ldots$ & 1800 & M7 pec & $\ldots$ \\
\hline 2MASS J20593353+7727266 & Gemini-North & GMOS & 2006 Aug 29 & $\ldots$ & 1800 & (bkg) & $\ldots$ \\
\hline 2MASS J21210987+6557255 & IRTF & SpeX & 2006 Sep 02 & Clear & 960 & $\ldots$ & (bkg) \\
\hline \multirow[t]{3}{*}{ 2MASS J21265916+7617440 } & Keck-II & NIRSPEC & 2005 Dec 09 & Clear & 1200 & $\ldots$ & T0 pec ${ }^{\mathrm{a}}$ \\
\hline & Keck-II & NIRSPEC & 2006 Aug 04 & Clear & 1200 & $\ldots$ & T0 pec \\
\hline & Subaru & FOCAS & 2007 Aug 20 & Clear & 3600 & L7 & $\ldots$ \\
\hline 2MASS J21403907+3655563 & IRTF & SpeX & 2006 May 31 & Clear & 480 & $\ldots$ & M8 pec \\
\hline 2MASS J21420390+2252290 & IRTF & SpeX & 2006 Sep 01 & Clear & 800 & $\ldots$ & WD \\
\hline \multirow[t]{6}{*}{ 2MASS J21481633+4003594 } & IRTF & SpeX & 2005 Sep 08 & Clear & 960 & $\ldots$ & L6.5 pec (red) \\
\hline & IRTF & SpeX & 2005 Sep 09 & Cirrus & 720 & $\ldots$ & L6.5 pec (red) \\
\hline & Keck-II & NIRSPEC & 2005 Dec 09 & Clear & 1200 & $\ldots$ & L6 pec ${ }^{\mathrm{a}}$ \\
\hline & Spitzer & IRS & 2005.956 & $(\mathrm{~N} / \mathrm{A})$ & 3904 & $\ldots$ & $\ldots$ \\
\hline & Keck-II & NIRSPEC & 2006 Aug 04 & Clear & 800 & $\ldots$ & L7 pec $\mathrm{e}^{\mathrm{e}}$ \\
\hline & Subaru & FOCAS & 2007 Aug 20/21 & Clear & 6000 & L6 & $\ldots$ \\
\hline \multirow{2}{*}{ 2MASS J21513979+3402444 } & IRTF & SpeX & 2005 Oct 20 & Clear & 960 & $\ldots$ & L6:: \\
\hline & IRTF & SpeX & 2008 Aug 28 & $\ldots$ & 1440 & $\ldots$ & L7 pec (sl. blue) \\
\hline 2MASS J21555848+2345307 & IRTF & SpeX & 2006 Sep 01 & Clear & 400 & $\ldots$ & L2 \\
\hline 2MASS J22021302-0228558 & IRTF & SpeX & 2005 Sep 08 & Clear & 960 & $\ldots$ & M8.5 \\
\hline \multirow{2}{*}{ 2MASS J22085600-1227448 } & Keck-II & NIRSPEC & $2005 \mathrm{Jul} 18$ & Clear & 1200 & $\ldots$ & $\mathrm{M}^{\mathrm{a}}$ \\
\hline & Magellan & LDSS3 & 2005 Oct 30 & Clear & 3000 & M9 & $\ldots$ \\
\hline \multirow[t]{2}{*}{ 2MASS J22114470+6856262 } & IRTF & SpeX & 2005 Sep 08 & Clear & 960 & $\ldots$ & L2 \\
\hline & IRTF & SpeX & 2005 Oct 20 & Clear & 960 & $\ldots$ & L2 \\
\hline \multirow[t]{2}{*}{ 2MASS J22120703+3430351 } & IRTF & SpeX & 2005 Oct 19 & Cirrus & 1440 & $\ldots$ & L6: \\
\hline & IRTF & SpeX & $2008 \mathrm{Jul} 12$ & Clear & 1200 & $\ldots$ & L6 \\
\hline 2MASS J22175686-4530532 & Magellan & LDSS3 & 2006 Aug 28 & Clear? & 3300 & M8 & $\ldots$ \\
\hline 2MASS J22210433-8905086 & Magellan & LDSS3 & 2006 Aug 28 & Clear? & 3000 & M6 & $\ldots$ \\
\hline 2MASS J22225588-4446197 & IRTF & SpeX & 2006 Aug 28 & lt. Cirrus & 960 & $\ldots$ & M8 \\
\hline 2MASS J22274034+1753215 & IRTF & SpeX & 2006 Sep 01 & Clear & 360 & $\ldots$ & WD \\
\hline 2MASS J23051335-7703177 & Magellan & LDSS3 & 2006 Aug 28 & Clear? & 1500 & M8 & $\ldots$ \\
\hline 2MASS J23092857+3246175 & IRTF & SpeX & 2005 Oct 18 & Cirrus & 960 & $\ldots$ & M8 \\
\hline \multirow[t]{2}{*}{ 2MASS J23174712-4838501 } & IRTF & SpeX & 2006 Sep 01 & Clear & 960 & $\ldots$ & L6.5 pec (red) \\
\hline & Magellan & LDSS3 & 2007 Aug 19 & $\ldots$ & & L4 pec & $\ldots$ \\
\hline \multirow[t]{2}{*}{ 2MASS J23294790-1607551 } & IRTF & SpeX & 2007 Jul 04 & 1t. Cirrus & 960 & $\ldots$ & M9 \\
\hline & Magellan & MagE & 2008 Nov 27 & Clear & 1800 & M9.5 & $\ldots$ \\
\hline 2MASS J23343177-1509294 & IRTF & SpeX & 2005 Sep 09 & Cirrus & 960 & $\ldots$ & M8.5 \\
\hline 2MASS J23343735-1646042A & IRTF & SpeX & 2006 Dec 20 & Clear & 720 & $\ldots$ & $<\mathrm{M} 3$ \\
\hline 2MASS J23343735-1646042B & IRTF & SpeX & 2006 Dec 20 & Clear & 960 & $\ldots$ & $<\mathrm{M} 3$ \\
\hline 2MASS J23355852-0013040 & IRTF & SpeX & 2006 Aug 28 & 1t. Cirrus & 960 & $\ldots$ & M8: \\
\hline 2MASS J23470713+0219127 & IRTF & SpeX & 2007 Oct 12 & Clear & 360 & $\ldots$ & $\mathrm{d} / \mathrm{sdM} 7$ \\
\hline 2MASS J23504023+7248408 & Gemini-North & GMOS & 2006 Aug 30 & $\ldots$ & 1800 & M5 (sl. pec?) & $\ldots$ \\
\hline \multirow{2}{*}{ 2MASS J23512200+3010540 } & IRTF & SpeX & 2005 Sep 08 & Clear & 1440 & $\ldots$ & L5 pec (red) \\
\hline & Magellan & LDSS3 & 2008 Oct 05 & Clear & 3600 & $\mathrm{~L} 5.5$ & $\ldots$ \\
\hline 2MASS J23531922+3656457 & IRTF & SpeX & 2006 Aug 17 & Clear & 720 & $\ldots$ & M8.5 \\
\hline
\end{tabular}

Notes. Additional spectroscopic data for 2MASS J1821+1414 and 2MASS J2148+4003 are presented in Looper et al. 2008.

a The near-infrared classification here is based only on a NIRSPEC N3 spectrum covering $1.15-1.35 \mu \mathrm{m}$.

b 2MASS J1043+1213: this discovery has since been independently confirmed and published by Chiu et al. (2006).

c 2MASS J1520-4422AB: near-infrared spectra types are taken from Burgasser et al. (2007).

d 2MASS J1924+5506: this shares common proper motion with an object $10^{\prime \prime} 0$ distant and at P.A. $=115^{\circ}$. This other object has a 960 sec IRTF-SpeX spectrum from 2005 Oct 19 (UT) that shows it to be a white dwarf.

e 2MASS J2148+4003: spectral type based on a NIRSPEC N1+N2 spectrum covering 0.95-1.30 $\mu \mathrm{m}$.

f 2MASS J0115-7519: this object was found in both the $1 \mathrm{X}-6 \mathrm{X}$ and the $1 \mathrm{X}-1 \mathrm{X}$ survey.

g 2MASS J0100+1711: this object is also known as LHS 1174. 
are $1 \mathrm{X}$ measurements taken from the 2MASS All-Sky Point Source Catalog for whichever epoch had the smaller overall uncertainties. $^{21}$ Column 7 lists the reduced proper motion at $J$ band, defined as $H_{J}=J+5 \log (\mu)+5$, computed using the proper motion $(\mu)$ and $J$ magnitude reported in Columns 5 and 8 , respectively.

Additional data for these proper motion candidates were gleaned from other sources. A 3 arcsec position around each 2MASS source was searched for a corresponding entry in the USNO-B1.0 Catalog (Monet et al. 2003). For sources with USNO-B matches, information for the nearest such match is listed in Columns 12-18. The total USNO-B-measured proper motion, error, and position angle are listed in Columns 12-13. Columns 14-18 give the B1, B2, R1, R2, and I magnitudes, respectively. Dual values of the $B$ and $R$ photometry may exist because the photographic survey material covers two epochs of the sky at both of these bands. Errors on the photographic magnitudes are generally $\sim 0.3 \mathrm{mag}$ (Monet et al. 2003). When doing the positional cross matching, we chose not to proper move the 2MASS PSC values to epoch and equinox J2000.0 because the epoch of the 2MASS measures is near J2000.0 anyway (2MASS surveyed the sky over the years 1997-2001) and the positions in the USNO-B Catalog are proper moved to epoch and equinox J2000.0 already. As a result, the only 2MASS proper motion candidates that lack a counterpart within our 3 arcsec radius are those that are too faint to be detected in the optical, those that have substantial motions $\left(>1^{\prime \prime} \mathrm{yr}^{-1}\right)$ that will have moved them beyond our search radius in the $\sim 3$ years between the 2MASS images and the given USNO-B J2000.0 positions, or those with problematic USNO-B measurements (blends, sources in crowded fields, etc.).

Finally, Columns 19-22 report SIMBAD ${ }^{22}$ info on each object, current as of mid-2008, if a SIMBAD match was found within 10 arcsec of the 2MASS PSC position. This larger match radius was chosen because positional info in SIMBAD comes from a variety of sources and could represent an epoch far removed (as much as 50 years) from the 2MASS position. Column 19 contains the SIMBAD-reported value for proper motion and error, and Column 20 contains the computed position angle of the motion vector. Column 21 gives the name of the object as listed in SIMBAD, and Column 22 gives the SIMBADreported spectral type.

The total number of unique proper motion candidates resulting from the searches above is 2778. Thirteen objects-2MASS J01153252-7519079, 2MASS J01182974-7504544, 2MASS J03431558+2354453, 2MASS J04112809-6859167, 2MASS J04212447-5954026, 2MASS J05205071-7755133, 2MASS J05254550-7425263, 2MASS J06195065-5952247, 2MASS J07025026-6102482, 2MASS J13271966-3110394, 2MASS J13273959-3551009, 2MASS J13375084-3549174, and 2MASS J13275892-3327329-were discovered in both the $1 \mathrm{X}-6 \mathrm{X}$ and the $1 \mathrm{X}-1 \mathrm{X}$ surveys. Of the 2778 unique objects, 647 lacked SIMBAD entries, 332 were not found within 3".0 of any source in the USNO-B Catalog, and 107 were 2MASS-only detections.

\footnotetext{
21 Photometry from epoch 1 would be selected if at least two of the following conditions were met in the 2MASS All-Sky Point Source Catalog:

$j \_$msigcom at epoch $1<j \_$msigcom at epoch $2, h \_$msigcom at epoch 1 $<h \_m s i g c o m$ at epoch $2, k \_$msigcom at epoch $1<k \_$msigcom at epoch 2 . Otherwise, photometry from epoch 2 is reported instead.

22 See http://simbad.u-strasbg.fr/simbad/.
}

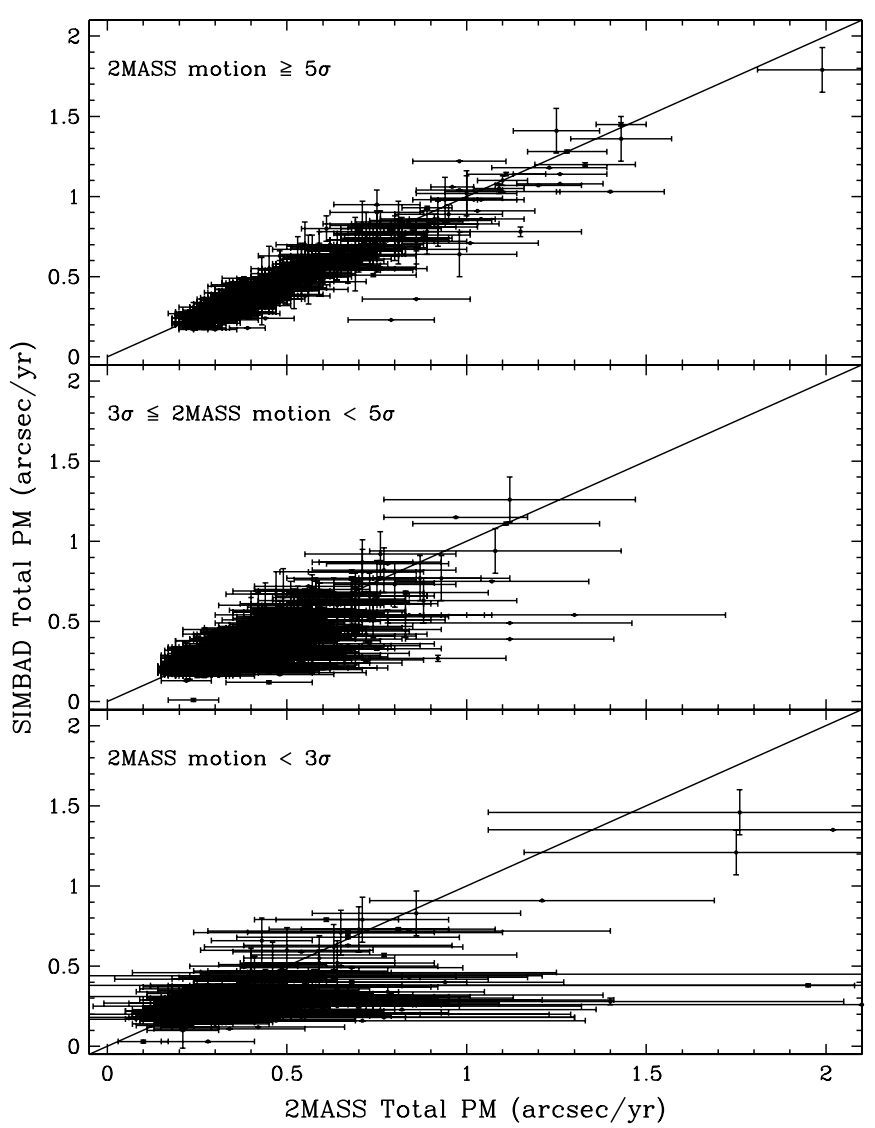

Figure 3. Comparison of $2 \mathrm{MASS}$ derived proper motions to those listed at SIMBAD.

\subsection{Diagnostic Plots}

\subsubsection{Judging the Robustness of the 2MASS Measures}

We can use the tabulated data in Table 1 to judge the credibility of our 2MASS-measured motions. In Figure 3, we compare the 2MASS motions to independent measurements from SIMBAD and find an excellent correlation for those objects whose motions are the most significant ( $>5 \sigma$, top panel). The correlation is not as tight for objects having 2MASS motions of smaller significance (bottom two panels) as one would expect.

Despite the excellent correlation in the top panel, it is important to understand why a few outliers still exist. One of these, 2MASS J19570113-5917285 has a 2MASS-measured motion of $\mu=0.79 \pm 0$.' $12 \mathrm{yr}^{-1}$, whereas the SIMBAD value is $\mu=0.23 \mathrm{yr}^{-1}$. Another outlier, 2MASS J02532235+0608003, has a 2MASS-measured motion of $\mu=0$ '. $86 \pm 0.15 \mathrm{yr}^{-1}$, whereas the SIMBAD value is $\mu=0.36 \mathrm{yr}^{-1}$.

Measuring the motion of 2MASS J1957-5917 using the 20 year baseline between the DSS images and 2MASS, we find a motion of $\mu \sim 0^{\prime \prime} .20 \mathrm{yr}^{-1}$, in agreement with the SIMBAD value. Hence, the 2MASS-measured motion appears to be in error. 2MASS J1957-5917 is fairly bright (7.0-8.0 mag in $J H K_{S}$ ) and stars in this brightness range are faint in the 2MASS $1 \mathrm{X}$ $51 \mathrm{~ms}$ Read1 exposures but nearly saturated in the $1.3 \mathrm{~s}$ Read2Read 1 exposures. For such objects, the astrometry can be less reliable than for stars just brighter (which have higher signalto-noise ratio $(\mathrm{S} / \mathrm{N})$ and are more robustly measured on the Read1 frames) or just dimmer (which are farther from saturation and easier to centroid in the Read2-Read1 frames). Thus, the 2MASS astrometry needs to be carefully scrutinized for objects in this near-infrared brightness range. 


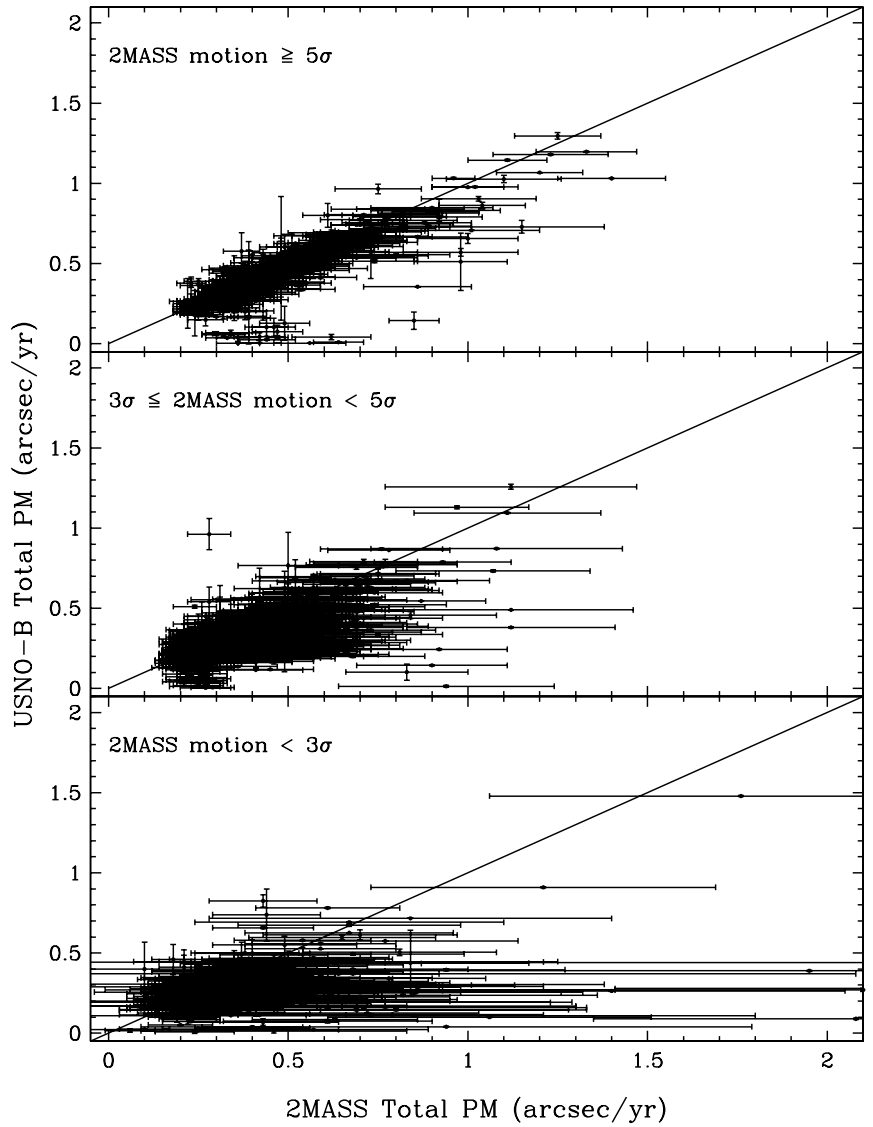

Figure 4. Comparison of $2 \mathrm{MASS}$ derived proper motions to those listed in the USNO-B Catalog.

In the case of 2MASS J0253+0608, positional measures using the 45 year baseline between the earliest DSS images and 2MASS suggest a motion of $\mu \sim 0$.'37 $\mathrm{yr}^{-1}$, also in agreement with the SIMBAD value. The two 2MASS imaging epochs are separated by only nine months, which means that an additional positional difference of $\sim 0$ '. 4 is being introduced between the 2MASS images. In this case, a study of a few bright 2MASS stars in the vicinity of this source show a $\sim 0$ '. 3 difference between the reconstructed positions of the two epochs. 2MASS J0253+0608 is located near the end of a six-degree-long 2MASS tile, and it appears that the astrometric solution for one of the two scans (scan 037 on 991217n) wandered off slightly, creating a $\sim 0^{\prime \prime} .3$ systematic error at that epoch. It is only because this particular object shows appreciable, real motion between the DSS and 2MASS images that it survived our by-eye checks.

Figure 4 shows the proper motion from the USNO-B Catalog plotted against the 2MASS-measured value. There is more scatter in the correlations here than in Figure 3 but this is to be expected since the USNO-B motions are measured in bulk and those from SIMBAD are generally well vetted. In general, the discrepancies here are caused by spurious measurements in the USNO-B. One example of this, from the upper panel of the figure, is 2MASS J06105984-6512200 (LHS 1832) whose 2MASS motion of $\mu=0.85 \pm 0.07 \mathrm{yr}^{-1}$ and USNO-B motion of $\mu=0^{\prime} .144 \pm 00^{\prime \prime} 054 \mathrm{yr}^{-1}$ are in clear disagreement. SIMBAD lists this object as having $\mu=0$ '. $78 \pm 0.0^{\prime} 01 \mathrm{yr}^{-1}$, agreeing within $1 \sigma$ of the 2MASS measurement. The USNO-B measurement is presumably the result of an incorrect association across epochs because this is a very crowded field in the outskirts of the Large Magellanic Cloud.

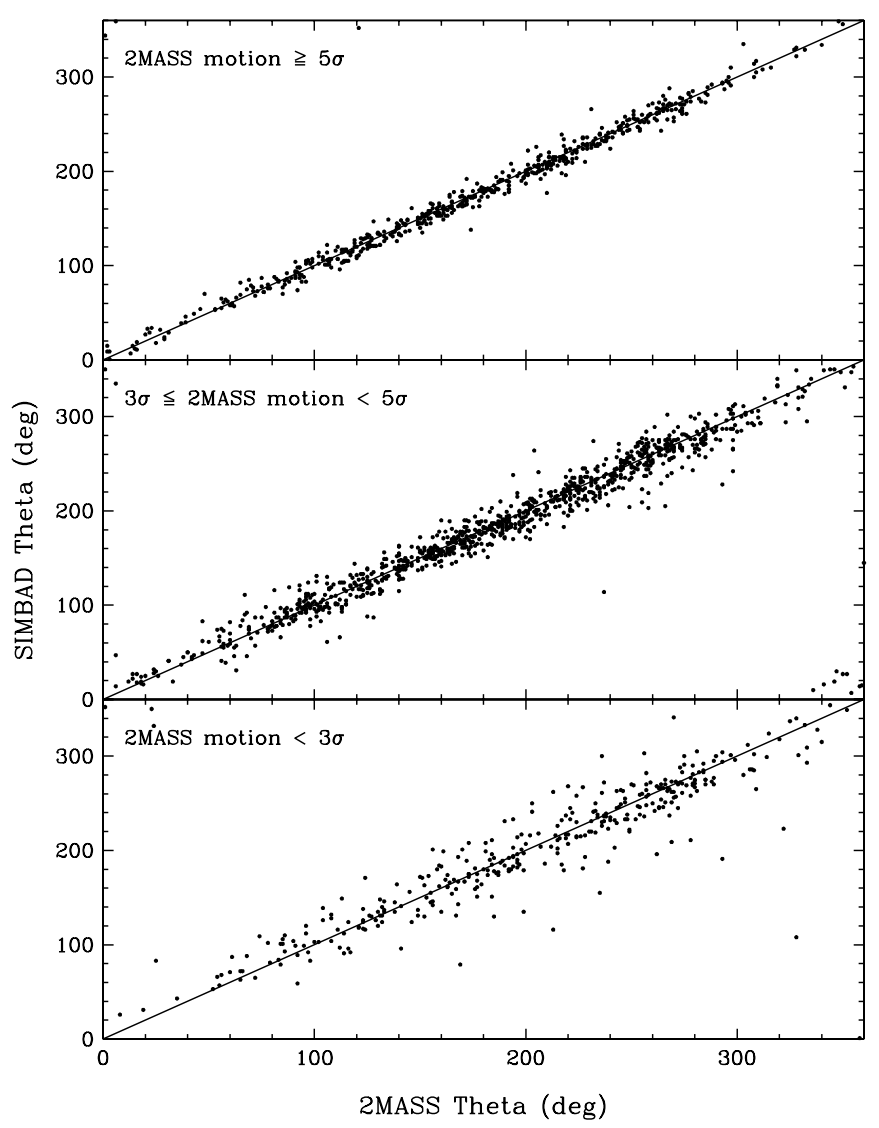

Figure 5. Comparison of 2MASS derived proper motion thetas to those listed at SIMBAD.

A similar situation holds true for the star 2MASS J01132818-7056507, whose discrepancy is obvious on the middle panel of Figure 4. This is the brighter component of the common proper motion pair CCDM J01135-7057AB, which lies in another crowded field, this time in the outskirts of the Small Magellanic Cloud. 2MASS measures identical motions of $\mu=0.28 \pm 0.06 \mathrm{yr}^{-1}$ for the two objects, whereas USNO-B reports non-identical and much higher values of $\mu=0.962 \pm 0.097 \mathrm{yr}^{-1}$ and $\mu=0.541 \pm 0.092 \mathrm{yr}^{-1}$ for the A and B components, respectively. Comparison of the DSS and 2MASS images, which represent a 23 year baseline, confirm the 2MASS measures.

Figures 5 and 6 show the correlation between measurements of the position angle. As with the two previous figures, these show an excellent correlation for those objects with 2MASS motions $>5 \sigma$, with the USNO-B agreement again being less tight than that of SIMBAD. The top panel of Figure 5 shows only one highly discrepant point, that of 2MASS J02192807-1938416, a previously known L1 dwarf whose 2MASS measurement of $\theta=121^{\circ}$ disagrees with the SIMBAD value of $\theta=352^{\circ}$, which itself appears to originate from the USNO-B Catalog. A comparison of the DSS $R$ - and $I$-band images with 2MASS images shows that the 2MASS value appears to be correct, the USNO-B value having been calculated from optical data with lower $S / N$.

These plots show that the 2MASS-measured proper motions agree well with published values. Nonetheless, like any other catalog derived from bulk-measured motions, some problems persist so users are advised to use caution and to perform their own quality assurance of the results before proceeding with analysis or follow-up. 


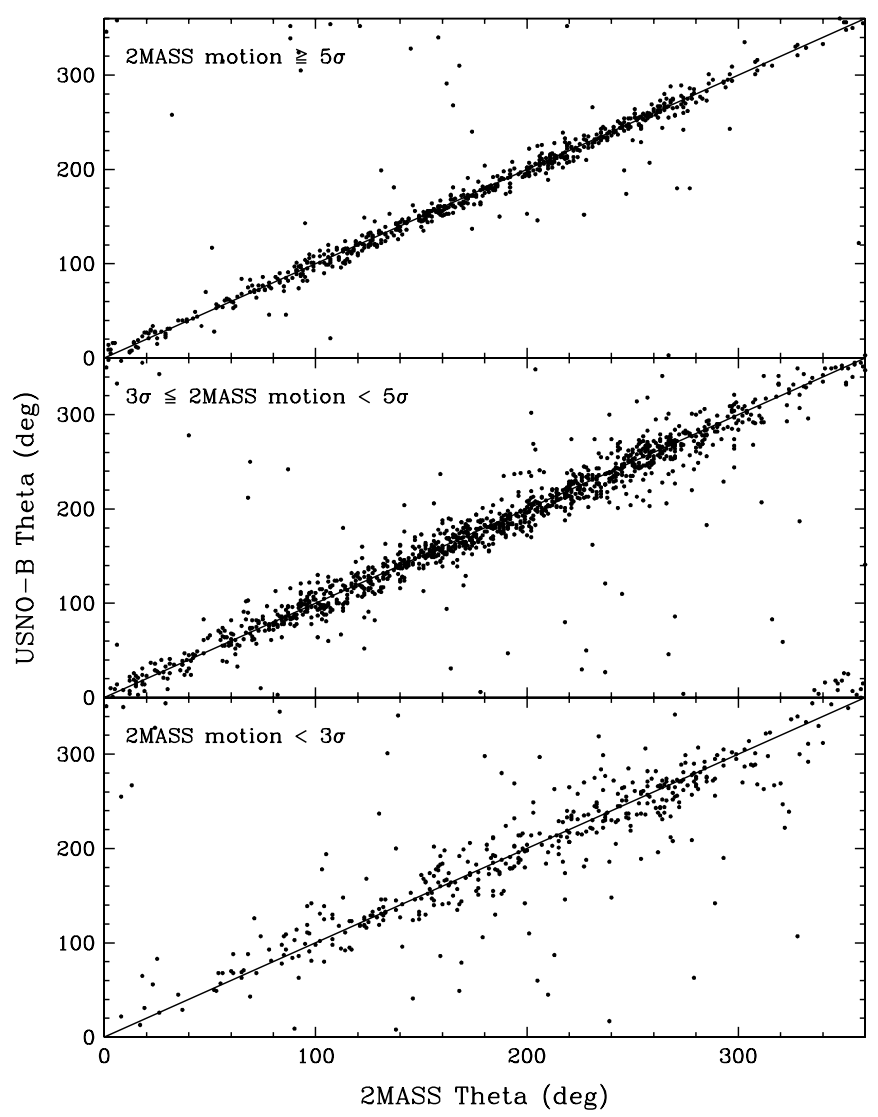

Figure 6. Comparison of $2 \mathrm{MASS}$ derived proper motion thetas to those listed in the USNO-B Catalog.

\subsubsection{Selecting the Choicest Sources}

On average, objects closer to the Sun should have larger proper motions than those more distant, so proper motion should be proportional to trigonometric parallax. With this in mind, Luyten (1925) substituted proper motion, $\mu$, for parallax, $\pi$, in the equation for distance modulus and invented a quantity, $H$, which he referred to as the reduced proper motion. This is defined by $H_{m}=m+5 \log (\mu)-5$, where $m$ is a particular photometric band such as 2MASS $J$. The quantity $H$ can be used to create a plot much like an H-R diagram, which can then be used to select potentially nearby stars for further followup. However, stars of higher than average kinematics will have artificially faint values of $H$, making them also stand out on such a diagram. Thus, reduced proper motion is also a valuable quantity for selecting potentially old objects for further followup. Figure 7 shows $H_{J}$, the reduced proper motion at $J$ band, plotted against $J-K_{s}$ color for objects in the 2MASS proper motion sample.

The $H_{J}$ versus $J-K_{s}$ diagram has several distinctive features. First, main-sequence stars earlier than late-K have $J-K_{s}<0.8$ and small values of $H_{J}$ whereas dwarfs later than mid-M have $J-K_{s}>0.9$ and larger values of $H_{J}$. Dwarfs between late-K and mid-M have nearly identical colors $\left(0.8<J-K_{s}<0.9\right)$ and fall at a wide range of $H_{J}$ bridging the earlier dwarfs in the upper left and the late-M and L dwarfs in the lower right. White dwarfs fall in the lower left portion because they tend to have blue near-infrared colors but are intrinsically faint for their motion (large $H_{J}$ ). T dwarfs, which have a wide range of near-infrared color $\left(-1<J-K_{s}<2\right)$ but are intrinsically faint (large $H_{J}$ ), span the entire lower portion of the diagram. Subdwarfs tend to be bluer in the near-infrared color than their

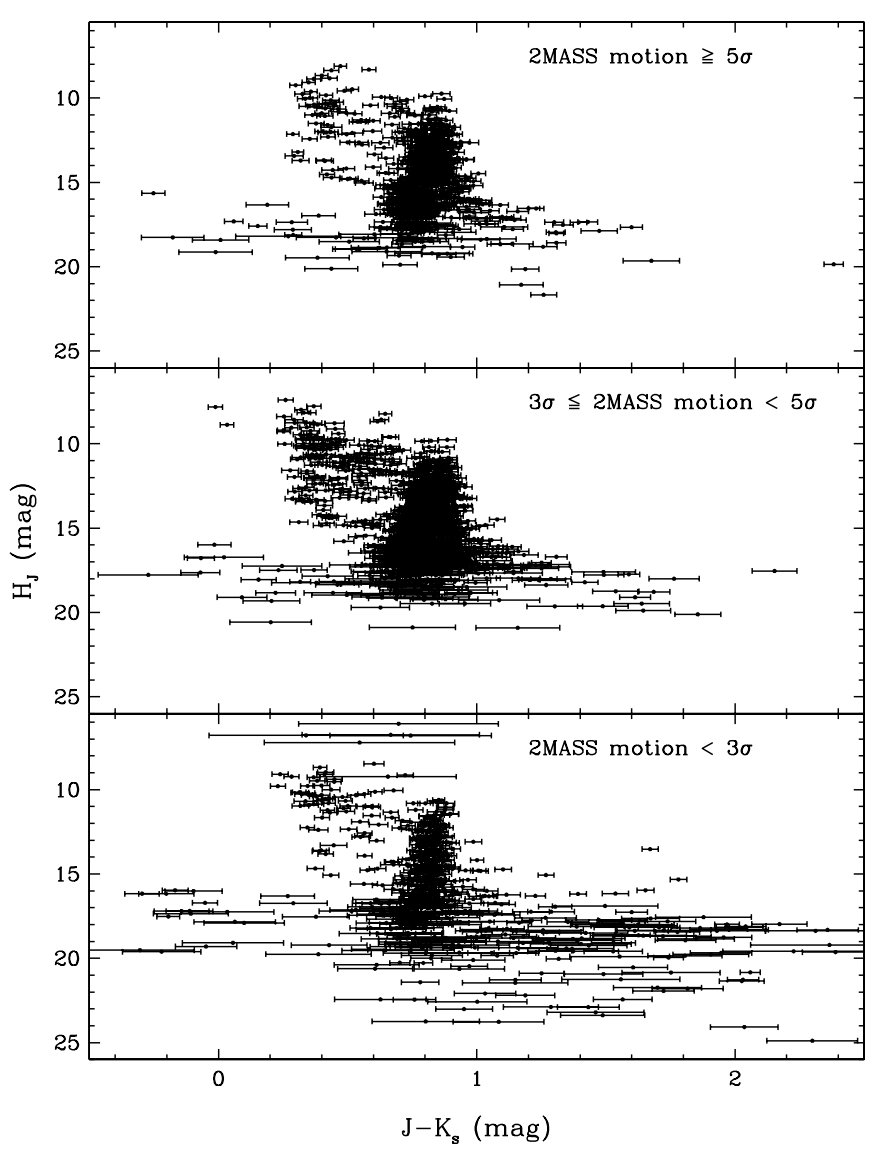

Figure 7. Reduced proper motion at $J$ band $\left(\right.$ aka $\left.H_{J}\right)$ vs. $J-K_{s}$ color for 2 MASS PM discoveries at various confidence-of-motion values. As described further in the text, the spectral sequence from pre-K to late-L begins in the upper left of the diagram, extends down the center of the figure at $0.8<J-K_{s}<0.9$, and continues toward the lower right. T dwarfs, which have a wide range of near-infrared color $\left(-1<J-K_{s}<2\right)$ but are intrinsically faint (large $H_{J}$ ), span the entire lower portion of the diagram. White dwarfs fall at lower left because they tend to have blue near-infrared colors but are intrinsically faint for their motion (large $H_{J}$ ). Old, low-metallicity objects (the subdwarfs) are pushed down and to the left relative to normal field objects because of their bluer colors and higher relative motions. Many of the objects with $0.6<J-K_{s}<0.9$ and $H_{J}>15$ are M subdwarfs falling below and to the left of the main population of field M dwarfs.

higher metallicity counterparts and because they are older tend to have higher motions as well. This pushes them down and to the left relative to normal field objects. The reason that the concentration of objects found with $0.6<J-K_{s}<0.9$ tends to show a blueward trend with increasing $H_{J}$ is because $\mathrm{M}$ subdwarfs dominate in the blueward extension stretching to larger $H_{J}$ values.

We have used Figure 7 to select subdwarf candidates and nearby, late-type candidates for spectroscopic study. Color-color diagrams can also be used as further diagnostics for candidate selection. One example is shown in Figure 8, which plots 2MASS $J-H$ versus $H-K_{s}$ color, useful for selecting $\mathrm{L}$ dwarfs (upper right quadrant of each panel) or midto late-T dwarfs and white dwarfs (lower left quadrant of each panel). The diagnostic ability of these reduced proper motion and color-color plots will be discussed further after the discussion of the spectroscopic follow-up below.

\section{SPECTROSCOPIC OBSERVATIONS}

Spectroscopic observations of 189 targets were obtained using eleven different optical/near-infrared instruments at eight 


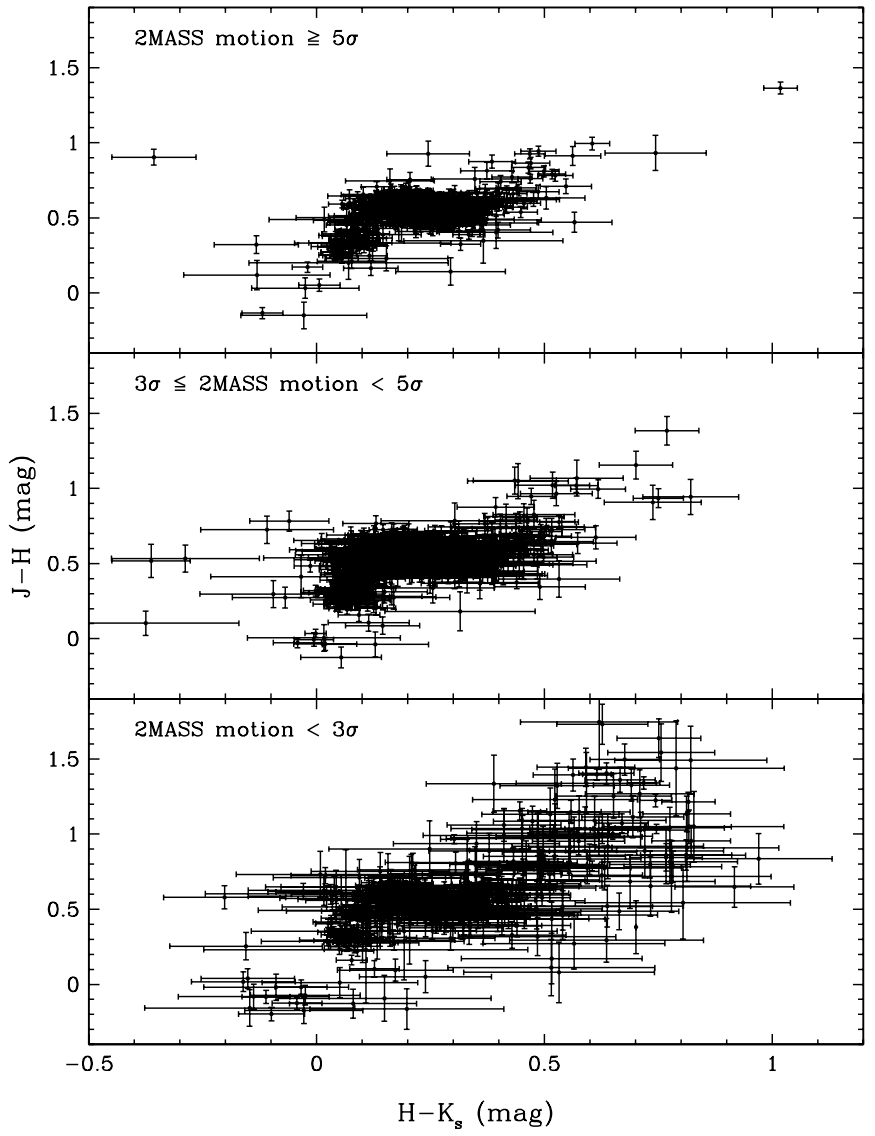

Figure 8. $J-H$ vs. $H-K_{s}$ color-color plot of all 2MASS proper motion sources. This figure can be compared to Figure 39, which shows the location of field dwarfs of spectral type B to L.

different observatories. Details of this follow-up are given in Table 2. This table gives the object's 2MASS PSC designation in Column 1, along with telescope and instrument used, the UT date of observation, weather conditions, and integration time in Columns 2-6. The spectral classifications, which are discussed in detail later, are given in Column 7 or Column 8. In addition, supporting calibration observations of a suite of $\mathrm{M}$ and $\mathrm{L}$ dwarfs and subdwarfs were also acquired, as listed in Table 3. Details of the observing setups and reductions are given below.

\subsection{Optical \\ 4.1.1. Magellan/LDSS3}

We obtained optical spectroscopy for sources in the southern hemisphere using the Low Dispersion Survey Spectrograph (LDSS-3) mounted on the Magellan $6.5 \mathrm{~m}$ Clay Telescope. LDSS-3 is an imaging spectrograph, upgraded by M. Gladders from the original LDSS-2 (Allington-Smith et al. 1994) for improved red sensitivity. We employed the VPH-red grism (660 line $\mathrm{mm}^{-1}$ ) with a 0.75 -wide long slit to obtain $6050-10500 \AA$ spectra across the entire chip with an average resolution of $4.5 \AA$. The OG590 longpass filter was used to eliminate second-order light shortward of $5900 \AA$. Two long exposures were obtained for each target, followed immediately by a series of helium/ neon/argon arc lamp and flat-field quartz lamp exposures.

Data were reduced in the $\operatorname{IRAF}^{23}$ environment. Raw science images were trimmed and subtracted by a median com-

\footnotetext{
23 IRAF is distributed by the National Optical Astronomy Observatory, which is operated by the Association of Universities for Research in Astronomy, Inc., under cooperative agreement with the National Science Foundation.
}

bined set of bias frames. The resulting images were divided by the corresponding normalized, median-combined, and biassubtracted set of flat-field frames. Spectra were then extracted using the apall task with background subtraction but without variance weighting (i.e., not "optimal extraction"). The dispersion solution for each target was determined using the tasks refspec, identify, and dispcor, and arc lamp spectra extracted using the same dispersion trace; solutions were typically accurate to 0.05 pixels, or $0.07 \AA$. Flux calibration was determined using the tasks standard and sensfunc with observations of the spectral standards from Hamuy et al. (1994).

\subsubsection{Magellan/MagE}

The Magellan Echellette (MagE) Spectrograph (Marshall et al. 2008) on the Magellan Clay Telescope was also used for follow-up. MagE is a cross-dispersed, medium-resolution ( $R \sim 4100)$ optical spectrograph, spanning 3000 to $10500 \AA$. All observations employed the $0{ }^{\prime \prime} 7$ slit, corresponding to a dispersion of $\sim 22 \mathrm{~km} \mathrm{~s}^{-1}$. The instrument was aligned with the parallactic angle to avoid atmospheric dispersion. Each observation was followed by a thorium-argon arc lamp to provide wavelength calibration.

The data were reduced using a preliminary version of the MagE Spectral Extractor (MASE; Bochanski et al. 2009). MASE is an IDL data reduction pipeline and GUI, which contains the entire reduction and calibration process. Briefly, each frame is bias corrected and flat fielded. Wavelength calibration is determined from ThAr observations and followed by sky subtraction and extraction. The pipeline employs simultaneous B-spline fitting (Kelson 2003) to both the background and object to measure the spectrum. Finally, the orders are combined and a relative flux calibration is applied.

\subsubsection{Gemini-North/GMOS and Gemini-South/GMOS}

The Gemini Multi-object Spectrometer (GMOS; Hook et al. 2004) on the Gemini-North and Gemini-South Telescopes was used during queue observations taken as programs GS-2004BQ-12, GN-2006A-Q-70, GS-2006B-Q-59, and GN-2006B-Q76. Spectra of known late-type subdwarfs were also taken for comparison under program GN-2004B-Q-15 (see Table 3). Observations were made using the RG610 order-blocking filter and 400 line $\mathrm{mm}^{-1}$ grating blazed at $7640 \AA$. In both cases data cover the wavelength range from 6000 to $10000 \AA$. Two consecutive observations, with the central wavelength offset slightly between exposures, were taken of each target to provide complete wavelength coverage despite the array gap. For the 2004B programs the array was read out in a traditional mode whereas in subsequent semesters the array was read out in nodand-shuffle mode, the latter providing improved sky subtraction at longer wavelengths where the $\mathrm{OH}$ telluric lines are more plentiful. In all cases, a 0 '.75-wide slit was used and provided a resolution of $\sim 5.5 \AA$.

The GMOS package under the IRAF Gemini tools was used to reduce the data. Specific details on our reduction procedures, both for traditional and nod-and-shuffle GMOS data are given elsewhere ${ }^{24}$, but in summary the dark frames were subtracted and data flat fielded using the gsreduce task, then sky lines were subtracted using gnsskysub. All spectra were extracted using gsextract. Standard stars (e.g., LTT 1020 and Feige 34) were taken with the same instrumental setup and were used to flux calibrate using calibrate. Two objects, 2MASS proper

\footnotetext{
${ }^{24}$ See Kelle Cruz's homepage, currently located at http://KelleCruz.com.
} 
Table 3

Supporting Observations of $\mathrm{M}$ and L Dwarfs and Subdwarfs

\begin{tabular}{|c|c|c|c|c|c|c|c|c|c|}
\hline $\begin{array}{l}\text { 2MASS Designation } \\
\text { J2000 Coords } \\
(1)\end{array}$ & Other Name & Telescope & $\begin{array}{l}\text { Instr. } \\
\text { (4) }\end{array}$ & $\begin{array}{l}\text { Obs. Date } \\
\text { (UT) } \\
(5)\end{array}$ & Weather & $\begin{array}{l}\text { Int. } \\
(\mathrm{s}) \\
(7)\end{array}$ & $\begin{array}{c}\text { Opt. Sp. Ty. } \\
\text { (8) }\end{array}$ & $\begin{array}{l}\text { New Opt. } \\
\text { Sp. Type } \\
\text { (9) }\end{array}$ & $\begin{array}{l}\text { Adopted } \\
\text { Type } \\
(10)\end{array}$ \\
\hline 2MASS J00114208-2057129 & LHS 1035 & Gemini-North & GMOS & 2004 Oct 06 & Clear & 600 & sdM6 & $\mathrm{b}$ & $\mathrm{d} / \mathrm{sdM} 5$ \\
\hline 2MASS J00433134+2054316 & LHS 1135 & Gemini-North & GMOS & 2004 Oct 06 & Clear & 900 & sdM6.5 & b & $\mathrm{d} / \mathrm{sdM} 5$ \\
\hline 2MASS J01031971+6221557 & Gl 51 & Keck-II & DEIMOS & $2004 \mathrm{Jul} 05$ & Clear & 420 & M5 & $\cdots$ & M5 \\
\hline 2MASS J01572792+0116433 & LP 589-7 & Gemini-North & GMOS & 2004 Sep 12 & Clear & 1200 & esdM5 & esdM5.5 & esdM5.5 \\
\hline 2MASS J05103896+1924078 & LHS 1742a & Gemini-North & GMOS & 2004 Sep 13 & Clear & 1200 & esdM5.5 & esdM6.5 & esdM6.5 \\
\hline 2MASS J05325346+8246465 & $\ldots$ & Gemini-North & GMOS & 2004 Nov 12 & Clear & 3600 & (e?)sdL7 & $\ldots$ & (e?)sdL7 \\
\hline 2MASS J05585891-2903267 & APMPM J0559-2903 & Gemini-North & GMOS & 2004 Nov 08 & Clear & 1200 & esdM7 & esdM7.5 & $\operatorname{esdM} 7.5^{\mathrm{c}}$ \\
\hline 2MASS J08223369+1700199 & LSR J0822+1700 & Gemini-North & GMOS & 2004 Nov 08 & Clear & 2400 & esdM6.5 & usdM8 & usdM7.5 \\
\hline 2MASS J08305161+3612577 & LHS 2023 & Gemini-North & GMOS & 2004 Nov 09 & Clear & 1200 & esdM6 & esdM6.5 & esdM6.5 \\
\hline 2MASS J08535631-2446565 & LHS 2067 & Gemini-North & GMOS & 2004 Nov 10 & Clear & 600 & sdM5 & $\ldots{ }^{b}$ & d/sdM5 \\
\hline 2MASS J13004255+1912354 & $\ldots$ & Keck-II & DEIMOS & 2004 Jul 05 & Clear & 1800 & L1 & & $\mathrm{L} 1$ \\
\hline 2MASS J14213789+5627180 & LP 97-817 & Keck-II & DEIMOS & 2004 Jul 05 & Clear & 600 & sdM5 & & M5 \\
\hline 2MASS J14250510+7102097 & LSR J1425+7102 & Keck-II & DEIMOS & $2004 \mathrm{Jul} 05$ & Clear & 1800 & sdM8 & sdM8 & sdM8 \\
\hline 2MASS J14284323+3310391 & LHS 2924 & Keck-II & DEIMOS & $2004 \mathrm{Jul} 05$ & Clear & 1800 & M9 & & M9 \\
\hline 2MASS J14390030+1839385 & LHS 377 & Keck-II & DEIMOS & $2004 \mathrm{Jul} 05$ & Clear & 1200 & sdM7 & $b$ & $\mathrm{~d} / \mathrm{sdM} 5$ \\
\hline 2MASS J14562713+1755001 & LHS 3001 & Keck-II & DEIMOS & 2004 Jul 05 & Clear & 600 & M4.5 & $\ldots$ & M4.5 \\
\hline 2MASS J14562776+1755090 & LHS 3002 & Keck-II & DEIMOS & 2004 Jul 05 & Clear & 600 & M6 & $\ldots$ & M6 \\
\hline 2MASS J15074769-1627386 & $\ldots$ & Keck-II & DEIMOS & $2004 \mathrm{Jul} 05$ & Clear & 1800 & L5 & $\ldots$ & L5 \\
\hline 2MASS J15202946+1434391 & LHS 3061 & Keck-II & DEIMOS & $2004 \mathrm{Jul} 05$ & Clear & 1200 & esdM5.5 & esdM5.5 & esdM5.5 \\
\hline 2MASS J15454034-2036157 & LHS 407 & Keck-II & DEIMOS & $2004 \mathrm{Jul} 05$ & Clear & 1200 & sdM5 & sdM4.5 & sdM4.5 \\
\hline 2MASS J16102900-0040530 & LSR J1610-0040 & Keck-II & DEIMOS & $2004 \mathrm{Jul} 05$ & Clear & 900 & sdM? odd & $\ldots{ }^{b}$ & $\ldots{ }^{\mathrm{f}}$ \\
\hline 2MASS J16201478-0116398 & LHS 3181 & Keck-II & DEIMOS & 2004 Jul 05 & Clear & 720 & sdM5+? & $\operatorname{sdM} 2.5$ & $\operatorname{sdM} 2.5$ \\
\hline 2MASS J16243393+3951492 & LHS 3189 & Keck-II & DEIMOS & 2004 Jul 05 & Clear & 900 & sdM5 & & M4: \\
\hline 2MASS J16553529-0823401 & vB 8 & Keck-II & DEIMOS & $2004 \mathrm{Jul} 05$ & Clear & 600 & M7 & & M7 \\
\hline 2MASS J17312974+2721233 & $\ldots$ & Keck-II & DEIMOS & $2004 \mathrm{Jul} 05$ & Clear & 600 & L0 & & L0 \\
\hline 2MASS J19165762+0509021 & vB 10 & Keck-II & DEIMOS & 2004 Jul 05 & Clear & 600 & M8 & $\cdots$ & M8 \\
\hline 2MASS J20362165+5100051 & LSR J2036+5059 & Keck-II & DEIMOS & 2004 Jul 05 & Clear & 1800 & $\operatorname{sdM} 7.5$ & $\operatorname{sdM} 7.5$ & $\mathrm{sdM} 7.5$ \\
\hline 2MASS J21225632+3656001 & LSR J2122+3656 & Keck-II & DEIMOS & 2004 Jul 05 & Clear & 900 & esdM5 & esdM5.5 & esdM5.5 \\
\hline
\end{tabular}

Notes.

a Typed on the subdwarf classification system of Lépine et al. (2007) using the spectra obtained here.

${ }^{b}$ Does not warrant a subdwarf classification on the new Lépine et al. (2007) system.

c Established as the esdM7.5 standard in Lépine et al. (2007).

d Established as the usdM7.5 standard in Lépine et al. (2007).

e Established as the sdM8 standard in Lépine et al. (2007).

${ }^{\mathrm{f}}$ See Cushing \& Vacca (2006) for more information on the classification and interpretation of LSR J1610-0040.

motion candidate 2MASS J06453153-6646120 along with the known subdwarf 2MASS J05325346+8246465, were taken concurrently with the G0 dwarfs CPD-65 772 and BD+82 165 , respectively. This was done to correct the data for telluric absorption by interpolating across the telluric features in the $\mathrm{G}$ dwarf, multiplying the uncorrected $\mathrm{G}$ dwarf spectrum by the corrected version, then multiplying the result against the target spectrum.

\subsubsection{Subaru/FOCAS}

Other optical spectroscopic observations were carried out on 2007 August 20 and 21 (UT) at the Subaru Telescope on Mauna Kea, Hawai'i, using the Faint Object Camera And Spectrograph (FOCAS; Kashikawa et al. 2002). FOCAS was used with the $300 \mathrm{R}$ grating blazed at $7500 \AA$ and the S058 filter to block second-order light from wavelengths shorter than $5800 \AA$. The grating tilt was set so that the wavelength region from 5850 to $10250 \AA$ was covered. Use of a long slit of width $0{ }^{\prime} 8$ resulted in a resolution of $8.5 \AA$. Both nights were clear but the seeing was variable over the course of both nights. On 2007 August 20 the seeing varied from 0.5 to 2".5. On 2007 August 21 the seeing was somewhat more stable, ranging only from $0{ }^{\prime} 6$ to 1".5. Subaru employs an atmospheric dispersion corrector, so keeping the slit aligned with the parallactic angle was not necessary.

The data were reduced and calibrated using standard IRAF routines. The overscan region of the array was used for bias subtraction, and a median of five dome flats taken on the first night were used to normalize the response of the detector. Because of strong, broad spectral signatures in the flat-field lamps themselves, special care was needed during the flat-field correction step. The FOCAS chip is designed to be used with multi-object spectroscopy, but for our single-slit observations most of the detector area is not used or needed for sky subtraction. This allowed us to perform a block average (in the spatial dimension) on a much smaller $21 \times 3260$ pixel region of the median dome flat corresponding to the area where the primary target spectrum and sky subtraction region would fall in subsequent exposures. This block-averaged, one-dimensional, vertical slice encompasses the gross undulations of the dome flat in the spectral dimension, and we replicated this slice across the spatial dimension to create a two-dimensional image. This image was then divided back into the median flat to produce a map that contains only small-scale flat-field variations. This map was then normalized using the IRAF routine imsurfit and divided into all other data frames to remove the flat-field signature across the detector. 


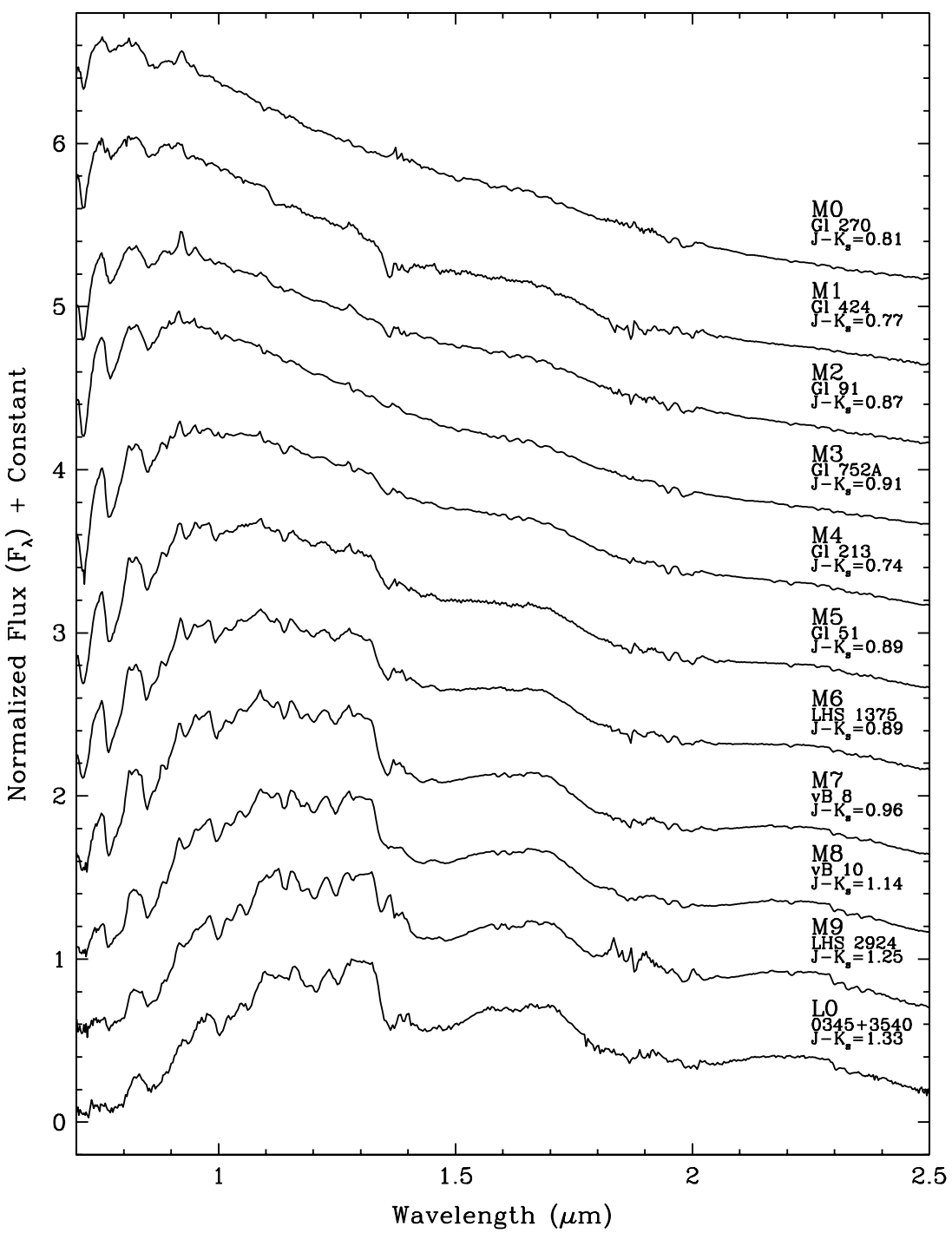

Figure 9. Standard dwarf spectral sequence covering 0.7 to $2.5 \mu \mathrm{m}$ for M0 through L0. All spectra were taken with SpeX in prism mode. Spectra have been normalized to one at $1.28 \mu \mathrm{m}$ and integer offsets added to separate the spectra vertically.

The individual stellar spectra were then extracted using the apall package. Wavelength calibration was achieved using arc lamps taken after each program object. Spectra were flux calibrated using an observation of LTT 9491 (Hamuy et al. 1994) taken on the first night. We checked this fluxing using another standard, Wolf 1346 (Massey \& Gronwall 1990), taken on the second night and the agreement was excellent. The G0 dwarfs SAO 103565, SAO 10140, and HD 207827 were acquired after the targets 2MASS J18212815+1414010, 2MASS $\mathrm{J} 21265916+7617440$, and 2MASS J21481633+4003594, respectively to remove the telluric bands of $\mathrm{O}_{2}$ at $6867-7000 \AA$ (the Fraunhofer B band) and 7594-7685 $\AA$ (the Fraunhofer A band) as well as $\mathrm{H}_{2} \mathrm{O}$ at $7186-7273,8162-8282$, and 〜8950-9650 A.

\subsubsection{Keck/LRIS}

Additional optical spectra were obtained with the Keck Low Resolution Imaging Spectrometer (LRIS, Oke et al. 1995) on the $10 \mathrm{~m} \mathrm{~W}$. M. Keck Observatory atop Mauna Kea, Hawaii. A 400 lines $\mathrm{mm}^{-1}$ grating blazed at $8500 \AA$ was used with a $1^{\prime \prime}$ slit and $2048 \times 2048$ CCD to produce $10 \AA$ resolution spectra covering the range $6300-10100 \AA$. The OG570 order-blocking filter was used to eliminate second-order light. The data were reduced and calibrated using standard IRAF routines. Flat-field exposures of the interior of the telescope dome were used to normalize the response of the detector.

Individual stellar spectra were extracted using the apextract routine in IRAF, allowing for the slight curvature of a pointsource spectrum viewed through the LRIS optics and using a template where necessary. Wavelength calibration was achieved using neon+argon arc lamp exposures taken after each program object. Finally, the spectra were flux calibrated using observations of standards from Hamuy et al. (1994).

\subsubsection{Keck/DEIMOS}

Other comparison spectra (Table 3) were obtained on 2004 July 5 (UT) using the Deep Imaging Multi-object Spectrograph (DEIMOS; Faber et al. 2003) at the $10 \mathrm{~m}$ Keck-II telescope. The instrument was used in single-object mode utilizing a $0 ! 7$ wide long slit. The 600 line $\mathrm{mm}^{-1}$ grating blazed at $7500 \AA$ was used along with a GG495 order-blocking filter to provide continuous wavelength coverage from 4950 to $10250 \AA$ at a resolution of $3.5 \AA$. Quartz lamps were used for flat fielding the detectors and a neon/argon/krypton lamp was used for wavelength calibration. The calibrator star Feige 56 (Hamuy et al. 1994) was used for flux calibration. Spectra were not 


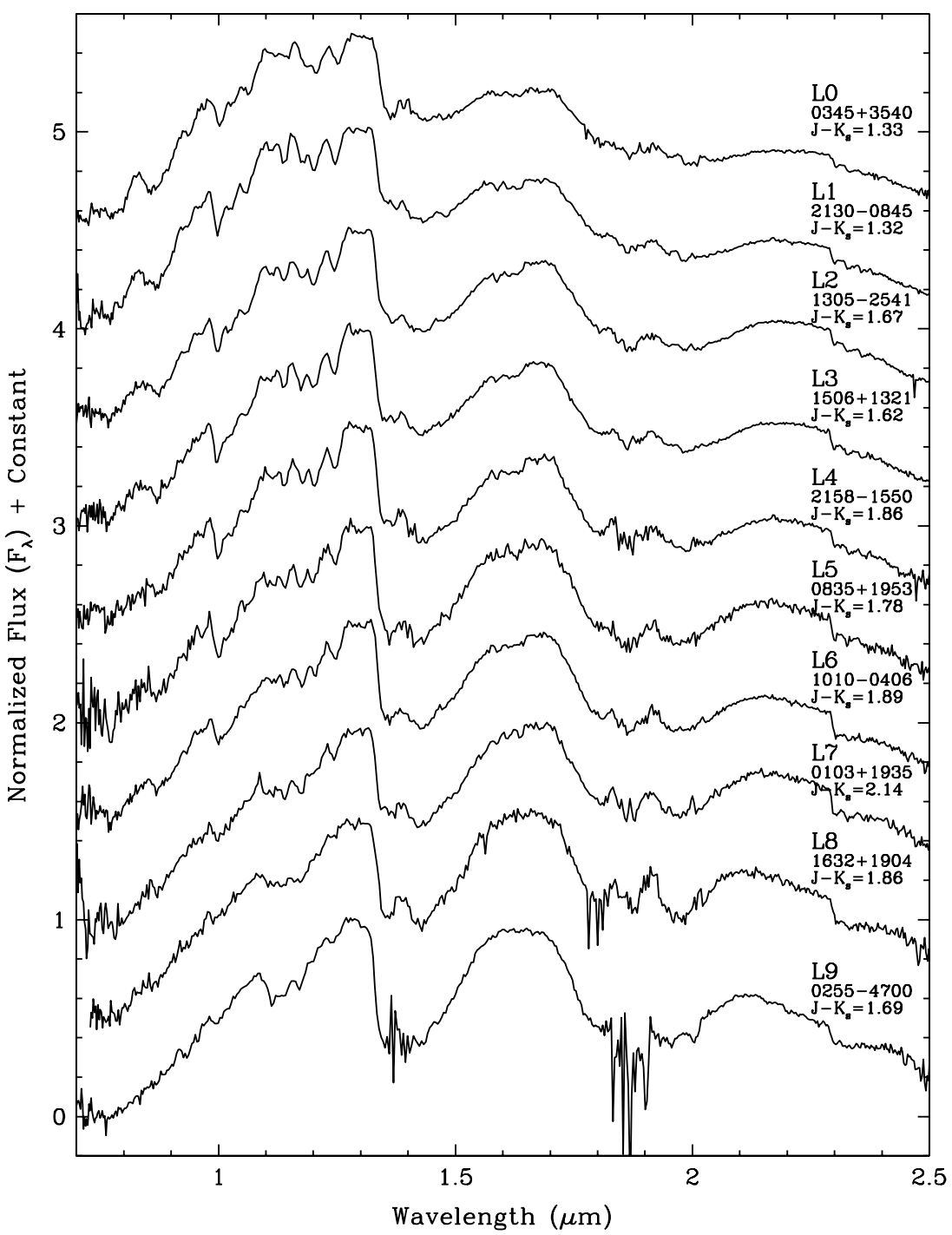

Figure 10. Standard dwarf spectral sequence covering 0.7 to $2.5 \mu \mathrm{m}$ for L0 through L9. All spectra were taken with SpeX in prism mode. Spectra have been normalized to one at $1.28 \mu \mathrm{m}$ and integer offsets added to separate the spectra vertically.

corrected for telluric absorption. Because DEIMOS was used in single-object mode, only two of the eight arrays in the mosaic contained science data. Standard procedures identical to those for the LRIS data above were used for data reduction.

\subsection{Infrared}

\subsubsection{IRTF/SpeX}

Most of our near-infrared spectra were obtained from the 3.0 m NASA Infrared Telescope Facility using SpeX (Rayner et al. 2003) in prism mode. Prism mode provided continuous coverage from $0.7-2.5 \mu \mathrm{m}$ in a single order on the $1024 \times 1024$ InSb array. Use of the 0.5 slit resulted in a resolving power of $R \approx 100$ in $J$ band to $\approx 300$ in $K$ band. For accurate sky subtraction, targets were observed in four nodded pairs generally having $120 \mathrm{~s}$ or $180 \mathrm{~s}$ integrations per position. Stars of spectral type $\mathrm{A} 0$ were observed immediately prior or after each target and at a similar airmass to provide flux calibration. Calibration flat fields were also acquired along with argon arc lamp exposures for wavelength calibration. Standard reductions were employed using the Spextool package, version 3.2 (Cushing et al. 2004; Vacca et al. 2003).

\subsubsection{Keck/NIRSPEC}

Additional spectra were obtained with the Near-Infrared Spectrometer (NIRSPEC; McLean et al. 1998, 2000) on the $10 \mathrm{~m}$ W. M. Keck Observatory. All objects were observed in the N3 configuration (see McLean et al. 2003) that, at low resolution, covers the portion of the $J$-band window from 1.14 to $1.36 \mu \mathrm{m}$ where the most diagnostic NIR features lie. Use of the $0^{\prime \prime} 38$ slit results in a resolving power of $R \equiv \lambda / \Delta \lambda \approx 2500$.

2MASS J21481633+4003594 was observed not only with the N3 configuration but also with the N1 and N2 configurations to provide full coverage from 0.95 to $1.36 \mu \mathrm{m}$. Two other targets-2MASS J17561080+2815238 and 2MASS J21265916+7617440-were observed in N1, N2, N3, N4, N6a, and N6b to cover the entire wavelength range from 0.94 to $2.35 \mu \mathrm{m}$. More information on the specifics of these instrument configurations can be found in McLean et al. (2003).

Data were obtained in two sets of dithered pairs, with typically a $300 \mathrm{~s}$ exposure obtained at each position. To measure telluric absorption and to calibrate the flux levels, dwarfs of type A0 were observed near in time and airmass to the target objects. Other calibrations consisted of neon and argon arc lamp spectra, a dark frame, and a spectrum of a flat-field lamp. We employed 


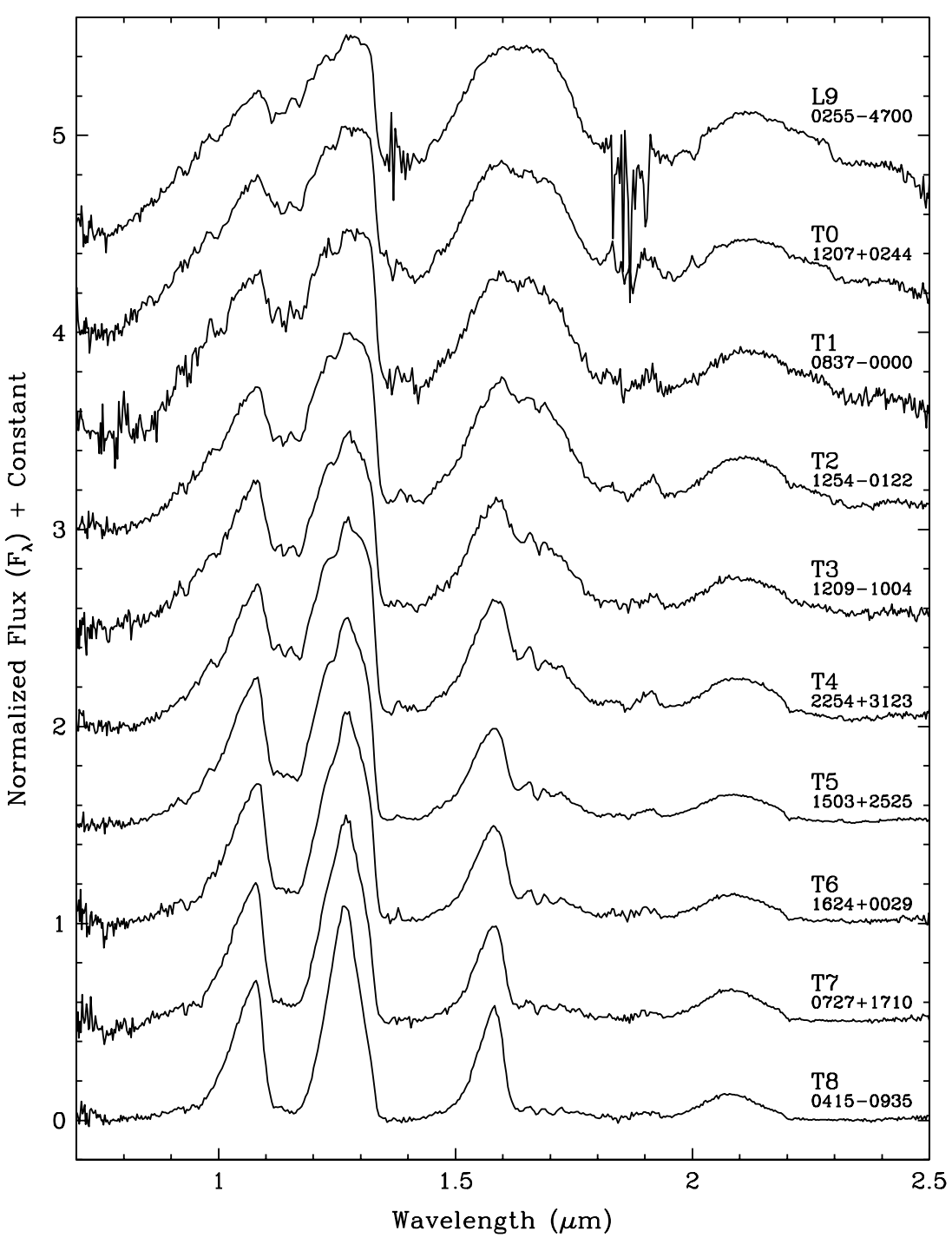

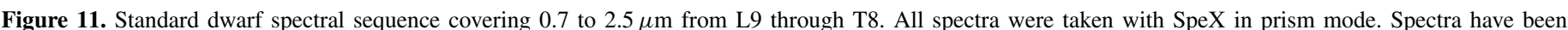

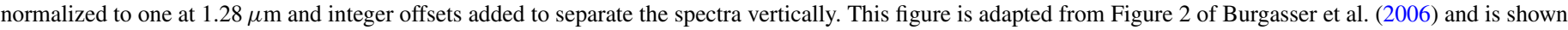
here to provide continuity with the $\mathrm{M}$ and $\mathrm{L}$ dwarf sequences in the previous two figures.

standard reductions using the IDL package, REDSPEC, as described in detail in McLean et al. (2003).

For the three objects with multiple wavelength coverages, spectra were stitched together using the overlaps between pieces. The N4 and N6a spectra almost touch but do not overlap in wavelength so a best guess was made to the normalization between the pieces. Synthetic photometry was produced for the final, stitched spectra and this photometry compared to actual values obtained in the 2MASS All-Sky Point Source Catalog. (Filter profiles for the 2MASS filters can be found in section VI.4.a of the 2MASS Explanatory Supplement.) The program sbands in IRAF was used to derive the fluxes in each bandpass. A comparison of the synthetic colors to the 2MASS $J-H, H-K_{s}$, and $J-K_{s}$ colors showed excellent agreement. All synthetic colors were typically within the $1 \sigma$ limits of the 2MASS measures, except for the $J-H$ color of 2MASS J21265916+7617440 (which was still within $2 \sigma$ ).

\subsubsection{SOAR/OSIRIS}

2MASS J0645-6646 was observed with the Ohio State Infrared Imager/Spectrometer (OSIRIS) mounted at the $4.1 \mathrm{~m}$
Southern Astrophysical Research Telescope (SOAR) located at Cerro Pachon, Chile. We used the 1".0 wide slit which provides a resolving power of $R \approx 1400$ across the $1.18-2.35 \mu \mathrm{m}$ wavelength range in three spectral orders. A series of nine $180 \mathrm{~s}$ integrations were obtained along the $24^{\prime \prime}$-long slit. The A0 dwarf HIP 33717 was also observed to correct for absorption due to Earth's atmosphere and to flux calibrate the final spectrum. The data were reduced using a modified version of the SpeXtool data reduction package (see IRTF/SpeX discussion, above). Wavelength calibration was accomplished using the $\mathrm{OH}$ airglow lines in the science frames. The spectra were then corrected for telluric absorption using the observations of the A0 V standard star via the method described in Vacca et al. (2003). The three spectral orders were then merged into a single spectrum covering the entire wavelength range.

\subsubsection{Spitzer/IRS}

Four targets-2MASS J11061191+2754215, 2MASS $\mathrm{J} 18212815+1414010,2 \mathrm{MASS} \mathrm{J} 18284076+1229207$, and 2MASS J21481633+4003594-were observed using the ShortLow (SL) module of the Infrared Spectrograph (IRS; Houck 
Table 4

New M and L Dwarfs Spectral Standards in the Near-infrared

\begin{tabular}{|c|c|c|c|c|c|c|c|}
\hline $\begin{array}{l}\text { Designation } \\
\text { J2000 coords } \\
\text { (1) }\end{array}$ & $\begin{array}{c}\text { Other Name } \\
\text { (2) }\end{array}$ & $\begin{array}{c}\text { Adopted Near-IR } \\
\text { Spec. Type } \\
\text { (3) }\end{array}$ & $\begin{array}{c}\text { 2MASS } J \\
\text { (mag) } \\
(4)\end{array}$ & $\begin{array}{c}\text { 2MASS } J-H \\
\text { (mag) } \\
(5)\end{array}$ & $\begin{array}{c}\text { 2MASS } J-K_{S} \\
(\mathrm{mag}) \\
(6)\end{array}$ & $\begin{array}{c}\text { Published Opt. } \\
\text { Spec. Ty. } \\
\text { (7) }\end{array}$ & $\begin{array}{l}\text { Optical } \\
\text { Ref. } \\
(8)\end{array}$ \\
\hline 2MASS J07193126+3249482 & G1 270 & M0 & $7.184 \pm 0.020$ & $0.643 \pm 0.039$ & $0.808 \pm 0.028$ & M0 & 3 \\
\hline 2MASS J11200526+6550470 & Gl 424 & M1 & $6.306 \pm 0.018$ & $0.576 \pm 0.027$ & $0.772 \pm 0.025$ & M1 & 8 \\
\hline 2MASS J02135359-3202282 & Gl 91 & M2 & $6.961 \pm 0.020$ & $0.634 \pm 0.033$ & $0.868 \pm 0.026$ & M2 & 8 \\
\hline 2MASS J19165526+0510086 & Gl 752A & M3 & $5.583 \pm 0.025$ & $0.654 \pm 0.040$ & $0.910 \pm 0.028$ & M3 & 3 \\
\hline 2MASS J05420897+1229252 & Gl 213 & M4 & $7.124 \pm 0.013$ & $0.497 \pm 0.028$ & $0.735 \pm 0.015$ & M4 & 3 \\
\hline 2MASS J01031971+6221557 & Gl 51 & M5 & $8.611 \pm 0.021$ & $0.597 \pm 0.035$ & $0.893 \pm 0.025$ & M5 & 3 \\
\hline 2MASS J02162977+1335136 & LHS 1375 & M6 & $9.871 \pm 0.021$ & $0.557 \pm 0.030$ & $0.890 \pm 0.021$ & M6 & 8 \\
\hline 2MASS J16553529-0823401 & vB 8 & M7 & $9.776 \pm 0.026$ & $0.575 \pm 0.038$ & $0.960 \pm 0.033$ & M7 & 3 \\
\hline 2MASS J19165762+0509021 & vB 10 & M8 & $9.908 \pm 0.021$ & $0.682 \pm 0.036$ & $1.143 \pm 0.030$ & M8 & 3 \\
\hline 2MASS J14284323+3310391 & LHS 2924 & M9 & $11.990 \pm 0.017$ & $0.765 \pm 0.036$ & $1.246 \pm 0.029$ & M9 & 3 \\
\hline 2MASS J03454316+2540233 & $\ldots$ & L0 & $13.997 \pm 0.027$ & $0.786 \pm 0.040$ & $1.325 \pm 0.036$ & L0 & 5 \\
\hline 2MASS J21304464-0845205 & $\ldots$ & L1 & $14.137 \pm 0.032$ & $0.803 \pm 0.045$ & $1.322 \pm 0.046$ & $\mathrm{~L} 1.5$ & 6 \\
\hline 2MASS J13054019-2541059 & Kelu-1AB & L2 & $13.414 \pm 0.026$ & $1.022 \pm 0.036$ & $1.667 \pm 0.035$ & L2 & 5 \\
\hline 2MASS J15065441+1321060 & $\ldots$ & L3 & $13.365 \pm 0.023$ & $0.985 \pm 0.031$ & $1.624 \pm 0.030$ & L3 & 7 \\
\hline 2MASS J21580457-1550098 & $\ldots$ & L4 & $15.040 \pm 0.040$ & $1.173 \pm 0.052$ & $1.855 \pm 0.054$ & L4: & 6 \\
\hline 2MASS J08350622+1953050 & $\cdots$ & L5 & $16.094 \pm 0.075$ & $1.205 \pm 0.094$ & $1.775 \pm 0.090$ & $\ldots$ & $\ldots$ \\
\hline 2MASS J10101480-0406499 & $\ldots$ & L6 & $15.508 \pm 0.059$ & $1.123 \pm 0.070$ & $1.889 \pm 0.075$ & L6 & 2 \\
\hline 2MASS J01033203+1935361 & $\ldots$ & L7 & $16.288 \pm 0.080$ & $1.391 \pm 0.098$ & $2.139 \pm 0.099$ & L6 & 1 \\
\hline 2MASS J16322911+1904407 & $\ldots$ & L8 & $15.867 \pm 0.070$ & $1.255 \pm 0.080$ & $1.864 \pm 0.084$ & L8 & 3 \\
\hline 2MASS J02550357-4700509 & $\ldots$ & L9 & $13.246 \pm 0.027$ & $1.042 \pm 0.036$ & $1.688 \pm 0.036$ & L8 & 6 \\
\hline
\end{tabular}

References. (1) Kirkpatrick et al. 2000; (2) Cruz et al. 2003; (3) Kirkpatrick et al. 1991; (4) Kirkpatrick et al. 1995; (5) Kirkpatrick et al. 1999; (6) Kirkpatrick et al. 2008; (7) Gizis et al. (2000); (8) unpublished from Kirkpatrick, as listed in DwarfArchives.org.

Table 5

New Low-gravity Objects

\begin{tabular}{cccccc}
\hline \hline $\begin{array}{c}\text { 2MASS Designation } \\
(1)\end{array}$ & $\begin{array}{c}\text { Opt. Sp. Ty. } \\
(2)\end{array}$ & $\begin{array}{c}\text { NIR Sp. Ty. } \\
(3)\end{array}$ & $\begin{array}{c}J-H \\
(4)\end{array}$ & $\begin{array}{c}H-K_{s} \\
(5)\end{array}$ & $J-K_{s}$ \\
2MASS J00040288-6410358 & L1 $\gamma$ & $\ldots$ & $0.955 \pm 0.103$ & $0.822 \pm 0.087$ & $1.777 \pm 0.113$ \\
2MASS J02340093-6442068 & L0 $\gamma$ & $\ldots$ & $0.883 \pm 0.089$ & $0.590 \pm 0.098$ & $1.473 \pm 0.093$ \\
2MASS J03032042-7312300 & L2 $\gamma$ & $\ldots$ & $1.041 \pm 0.137$ & $0.775 \pm 0.120$ & $1.816 \pm 0.137$ \\
2MASS J04062677-3812102 & L0 $\gamma$ & L1 pec & $1.057 \pm 0.162$ & $0.598 \pm 0.154$ & $1.655 \pm 0.172$ \\
2MASS J05341594-0631397 & M8 $\gamma$ & M8 pec & $0.685 \pm 0.124$ & $0.433 \pm 0.137$ & $1.118 \pm 0.124$ \\
2MASS J15575011-2952431 & M9 $\delta$ & M9 pec & $0.866 \pm 0.159$ & $0.605 \pm 0.153$ & $1.471 \pm 0.163$ \\
\hline
\end{tabular}

Notes. Optical spectral types use the Greek suffix designations of Cruz et al. (2009) in which $\gamma$ is used to denote low gravity (as opposed to normal ( $\alpha$, usually dropped for convenience) or intermediate $(\beta)$ gravity). Objects of extremely low gravity are further denoted by $\delta$, as originally outlined in Kirkpatrick (2005) and Kirkpatrick et al. (2006).

Table 6

New Red L Dwarfs

\begin{tabular}{cllccc}
\hline \hline \multicolumn{1}{c}{ 2MASS Designation } & Opt. Sp. Ty. & NIR Sp. Ty. & $J-H$ & $H-K_{s}$ & $J-K_{s}$ \\
$(1)$ & $(2)$ & \multicolumn{1}{c}{$(3)$} & $(4)$ & $(5)$ & $(6)$ \\
\hline 2MASS J13313310+3407583 & L0 & L1 pec (red) & $0.932 \pm 0.041$ & $0.516 \pm 0.038$ & $1.448 \pm 0.035$ \\
2MASS J18212815+1414010 & L4.5 & L5 pec (red) & $1.035 \pm 0.031$ & $0.746 \pm 0.028$ & $1.781 \pm 0.032$ \\
2MASS J21481633+4003594 & L6 & L6.5 pec (red) & $1.364 \pm 0.041$ & $1.018 \pm 0.038$ & $2.382 \pm 0.037$ \\
2MASS J23174712-4838501 & L4 pec & L6.5 pec (red) & $1.225 \pm 0.044$ & $0.744 \pm 0.036$ & $1.969 \pm 0.048$ \\
2MASS J23512200+3010540 & L5.5 & L5 pec (red) & $1.278 \pm 0.123$ & $0.553 \pm 0.093$ & $1.831 \pm 0.118$ \\
\hline
\end{tabular}

Note. ${ }^{a}$ See Looper et al. (2008) for in-depth discussion.

et al. 2004) on board the Spitzer Space Telescope. These were observed as Spitzer AORs 16201728, 16176128, 16202240, and 16201984 , respectively. The SL module covers $5.2-15.3 \mu \mathrm{m}$ at $R \approx 90$ in two orders. The observations consisted of a series of exposures taken at two different positions along each slit. The total exposure time for each target was 3904 s, except for 2MASS J1828+1229 which was observed for only $2562 \mathrm{~s}$. The raw data were processed with the IRS pipeline (version S13) at the Spitzer Science Center and were further reduced as described in Cushing et al. (2006). Briefly, the spectra were extracted with a fixed-width aperture of $6^{\prime \prime}$ and observations of $\alpha$ Lac obtained as part of the IRS calibration observations were used to remove the instrument response function and to flux calibrate.

\section{SPECTROSCOPIC CLASSIFICATION}

The spectroscopic data discussed above have been used to classify each object in the optical and/or near-infrared, 
Table 7

New M and L Subdwarfs

\begin{tabular}{|c|c|c|c|c|c|}
\hline $\begin{array}{c}\text { 2MASS Designation } \\
\text { (1) }\end{array}$ & $\begin{array}{c}\text { Opt. Sp. Ty. } \\
\text { (2) }\end{array}$ & $\begin{array}{l}\text { NIR Sp. Ty. } \\
\text { (3) }\end{array}$ & $\begin{array}{c}J-H \\
(4)\end{array}$ & $\begin{array}{c}H-K_{s} \\
\quad(5)\end{array}$ & $\begin{array}{c}J-K_{s} \\
(6)\end{array}$ \\
\hline 2MASS J00054517+0723423 & M4 & sdM6 & $0.467 \pm 0.072$ & $0.314 \pm 0.089$ & $0.781 \pm 0.072$ \\
\hline 2MASS J00524886+1712434 & $\ldots$ & esdM5 & $0.471 \pm 0.124$ & $0.180 \pm 0.156$ & $0.651 \pm 0.134$ \\
\hline 2MASS J00554279+1301043 & M6 & $\mathrm{d} / \mathrm{sdM} 7$ & $0.650 \pm 0.112$ & $0.332 \pm 0.124$ & $0.982 \pm 0.114$ \\
\hline 2MASS J03073402-1736375 & usdM2 & $\ldots$ & $0.313 \pm 0.112$ & $0.390 \pm 0.148$ & $0.703 \pm 0.132$ \\
\hline 2MASS J03440399+2730504 & sdM3.5 & $\ldots$ & $0.549 \pm 0.078$ & $0.227 \pm 0.100$ & $0.776 \pm 0.089$ \\
\hline 2MASS J04470652-1946392 & sdM7.5 & sdM7.5 & $0.230 \pm 0.084$ & $0.153 \pm 0.134$ & $0.383 \pm 0.123$ \\
\hline 2MASS J06211484+6558164 & esdM0 & $<$ esdM5 & $0.472 \pm 0.042$ & $0.199 \pm 0.040$ & $0.671 \pm 0.034$ \\
\hline 2MASS J06453153-6646120 & sdL8 & sdL8 & $0.874 \pm 0.044$ & $0.385 \pm 0.051$ & $1.259 \pm 0.050$ \\
\hline 2MASS J10462067+2354307 & $\ldots$ & sdM6 & $0.440 \pm 0.106$ & $0.166 \pm 0.156$ & $0.606 \pm 0.145$ \\
\hline 2MASS J11582077+0435014 & sdL 7 & sdL7 & $0.927 \pm 0.085$ & $0.245 \pm 0.091$ & $1.172 \pm 0.084$ \\
\hline 2MASS J14270666+4808164 & $\ldots$ & $\leqslant$ sdM5 & $0.455 \pm 0.064$ & $0.076 \pm 0.095$ & $0.531 \pm 0.090$ \\
\hline 2MASS J16130315+6502051 & $\ldots$ & sdM6 & $0.535 \pm 0.101$ & $0.256 \pm 0.113$ & $0.791 \pm 0.107$ \\
\hline 2MASS J17561080+2815238 & sdL1 & L1 pec (blue) & $0.577 \pm 0.053$ & $0.322 \pm 0.058$ & $0.899 \pm 0.053$ \\
\hline 2MASS J18355309-3217129 & M6.5 & $\mathrm{d} / \mathrm{sdM} 7$ & $0.368 \pm 0.101$ & $0.358 \pm 0.129$ & $0.726 \pm 0.105$ \\
\hline 2MASS J23470713+0219127 & $\ldots$ & $\mathrm{d} / \mathrm{sdM} 7$ & $0.489 \pm 0.040$ & $0.304 \pm 0.041$ & $0.793 \pm 0.036$ \\
\hline
\end{tabular}

Table 8

Known L Subdwarfs ${ }^{\mathrm{a}}$

\begin{tabular}{lccc}
\hline \hline $\begin{array}{c}\text { 2MASS Designation } \\
\text { J2000 Coords }\end{array}$ & Ref. & Opt. Type & NIR Type \\
$(1)$ & $(2)$ & $(3)$ & $(4)$ \\
\hline 2MASS J05325346+8246465 & 2 & $(\mathrm{e}$ ?)sdL7 & $(\mathrm{e}$ ?)sdL7 \\
2MASS J06164006-6407194 & 3 & sdL5 & sdL: \\
2MASS J06453153-6646120 & 1 & sdL8 & sdL8 \\
2MASS J11582077+0435014 & 1 & sdL7 & sdL7 \\
SDSS J125637.13-022452.4 & 4 & sdL3.5 & sdL3.5 \\
SDSS J141624.08+134826.7 & 6 & sdL7 & sdL7 \\
2MASS J16262034+3925190 & 5 & sdL4 & sdL4 \\
2MASS J17561080+2815238 & 1.05 & 0.08 \\
\hline
\end{tabular}

Notes.

a Another purported L subdwarf, ULAS J135058.86+081506.8, has been published by Lodieu et al. (2010), but the limited spectroscopic evidence cannot distinguish between an L subdwarf and a blue L dwarf.

b Both Schmidt et al. (2010) and Bowler et al. (2010) prefer to call this object a blue L dwarf rather than an L subdwarf. Although low-metallicity signatures are more subtle than in 2MASS J0532+8246, we believe that they still point to a subdwarf nature for this object.

References. (1) This paper; (2) Burgasser et al. 2003; (3) Cushing et al. 2009; (4) Sivarani et al. via Burgasser et al. 2007; (5) Burgasser 2004; (6) Schmidt et al. 2010 and Bowler et al. 2010.

Table 9

New Blue L Dwarfs

\begin{tabular}{|c|c|c|c|c|c|}
\hline $\begin{array}{c}\text { 2MASS Designation } \\
\text { (1) }\end{array}$ & $\begin{array}{l}\text { Opt. Sp. Ty. } \\
\text { (2) }\end{array}$ & $\begin{array}{l}\text { NIR Sp. Ty. } \\
\text { (3) }\end{array}$ & $\begin{array}{c}J-H \\
(4)\end{array}$ & $\begin{array}{c}H-K_{s} \\
(5)\end{array}$ & $\begin{array}{c}J-K_{s} \\
(6)\end{array}$ \\
\hline 2MASS J00150206+2959323 & L7 & L7.5 pec (blue) & $0.932 \pm 0.117$ & $0.744 \pm 0.111$ & $1.676 \pm 0.109$ \\
\hline 2MASS J03001631+2130205 & $\ldots$ & L6 pec (sl. blue) & $1.146 \pm 0.103$ & $0.420 \pm 0.091$ & $1.566 \pm 0.098$ \\
\hline 2MASS J10461875+4441149 & $\ldots$ & L5 pec (sl. blue) & $0.966 \pm 0.082$ & $0.526 \pm 0.079$ & $1.492 \pm 0.078$ \\
\hline 2MASS J11181292-0856106 & L6 & L6 pec (blue) & $0.970 \pm 0.107$ & $0.590 \pm 0.103$ & $1.560 \pm 0.102$ \\
\hline 2MASS J13023811+5650212 & $\mathrm{L} 2$ & L3: pec (sl. blue) & $0.891 \pm 0.160$ & $0.497 \pm 0.162$ & $1.388 \pm 0.173$ \\
\hline 2MASS J14403186-1303263 & L1 & L1 pec (sl. blue) & $0.660 \pm 0.096$ & $0.468 \pm 0.105$ & $1.128 \pm 0.082$ \\
\hline 2MASS J19495702+6222440 & L2 pec (blue) & L2 pec (sl. blue) & $0.889 \pm 0.181$ & $0.720 \pm 0.173$ & $1.609 \pm 0.167$ \\
\hline 2MASS J21513979+3402444 & $\ldots$ & L7 pec (sl. blue) & $0.908 \pm 0.224$ & $0.813 \pm 0.202$ & $1.721 \pm 0.187$ \\
\hline
\end{tabular}

depending upon the wavelength coverage. Although on average the optical and near-infrared types should agree, there will be cases where they are different. This should not be regarded as a problem with either classification. The two wavelength regimes sample different levels in the atmosphere, so a difference in spectral types provides important clues about the underlying physics. Differing spectral types may also indicate the presence of binarity. Objects with discrepant types are discussed further in Section 6.

\subsection{Optical Types: Comparing to Pre-existing Anchors}

We have typed the LDSS3, MagE, GMOS, FOCAS, LRIS, and DEIMOS spectra as follows. Each spectrum has been normalized to one at $8250 \AA$ and overplotted on a suite of like-normalized LRIS spectra of primary M, L, and T optical spectral standards from Kirkpatrick et al. (1991), Kirkpatrick et al. (1999), and Burgasser et al. (2003). These plots have then been examined by eye to determine the best match and to 
Table 10

Known Blue L Dwarfs

\begin{tabular}{|c|c|c|c|c|c|c|c|c|c|}
\hline $\begin{array}{l}\text { 2MASS Designation } \\
\text { J2000 Coords } \\
\text { (1) }\end{array}$ & $\begin{array}{l}\text { Discovery } \\
\text { Ref. } \\
\text { (2) }\end{array}$ & $\begin{array}{c}\mu \\
\left({ }^{\prime \prime} \mathrm{yr}^{-1}\right) \\
(3)\end{array}$ & $\begin{array}{l}\text { Dist. } \\
\text { (pc) } \\
(4)\end{array}$ & $\begin{array}{c}V_{\tan } \\
\left(\mathrm{km} \mathrm{s}^{-1}\right) \\
(5)\end{array}$ & $\begin{array}{c}\text { Kinematic } \\
\text { Ref. } \\
\text { (6) }\end{array}$ & $\begin{array}{l}\text { Opt. } \\
\text { Sp. Type } \\
(7)\end{array}$ & $\begin{array}{l}\text { Near-IR } \\
\text { Sp. Type } \\
(8)\end{array}$ & $\begin{array}{l}\text { Sp. Type } \\
\text { Ref. } \\
\text { (9) }\end{array}$ & $\begin{array}{c}\text { 2MASS } J-K_{S} \\
\text { (mag) } \\
(10)\end{array}$ \\
\hline 2MASS J00150206+2959323 & 1 & $0.50 \pm 0.09$ & 21: & $50 \pm 9$ & 1 & L7 & L7.5 pec (blue) & 1 & $1.676 \pm 0.109$ \\
\hline 2MASS J03001631+2130205 & 1 & $0.27 \pm 0.19$ & 22: & $28 \pm 20$ & 1 & $\ldots$ & L6 pec (sl. blue) & 1 & $1.566 \pm 0.098$ \\
\hline SDSS J080531.84+481233.0 & 2 & $0.46 \pm 0.02$ & $15 \pm 3$ & $32 \pm 6$ & 8 & L4 & $\mathrm{L} 9 \pm 1.5$ & 14,2 & $1.290 \pm 0.055$ \\
\hline SIPS J0921-2104 ${ }^{\mathrm{b}}$ & 6 & $0.94 \pm 0.02$ & $12 \pm 1$ & $56 \pm 4$ & 8 & $\mathrm{~L} 1.5$ & $\mathrm{~L} 4 \pm 1.5$ & 11,10 & $1.089 \pm 0.033$ \\
\hline SDSS J093109.56+032732.5 & 2 & $0.63 \pm 0.03$ & $36 \pm 8$ & $108 \pm 23$ & 8 & $\ldots$ & $\mathrm{L} 7.5 \pm 1.5$ & 2 & $\ldots$ \\
\hline SDSS J103321.92+400549.5 & 5 & $0.24 \pm 0.02$ & $45 \pm 8$ & $53 \pm 10$ & 8 & $\ldots$ & L6 & 5 & $\ldots$ \\
\hline 2MASS J10461875+4441149 & 1 & $0.27 \pm 0.07$ & 24: & $31 \pm 8$ & 1 & $\ldots$ & L5 pec (sl. blue) & 1 & $1.492 \pm 0.078$ \\
\hline 2MASS J11181292-0856106 & 1 & $0.59 \pm 0.14$ & 21: & $59 \pm 14$ & 1 & L6 & L6 pec (blue) & 1 & $1.560 \pm 0.101$ \\
\hline SDSS J112118.57+433246.5 & 5 & $0.06 \pm 0.04$ & $46 \pm 6$ & $14 \pm 6$ & 8 & $\ldots$ & $\mathrm{L} 7.5$ & 5 & $1.434 \pm 0.286$ \\
\hline 2MASS J11263991-5003550 & 4 & $1.63 \pm 0.01$ & $14 \pm 1$ & $106 \pm 11$ & 10 & L4.5 & $\mathrm{L} 6.5 \pm 2$ & 10 & $1.168 \pm 0.043$ \\
\hline 2MASS J13004255+1912354 & 13 & $1.49 \pm 0.03$ & $14 \pm 1$ & $99 \pm 7$ & 8 & L1 & L3 & 13,10 & $1.093 \pm 0.030$ \\
\hline 2MASS J13023811+5650212 & 1 & $0.33 \pm 0.27$ & 65: & $102 \pm 84$ & 1 & $\mathrm{~L} 2$ & L3: pec (sl. blue) & 1 & $1.388 \pm 0.173$ \\
\hline SDSS J133148.92-011651.4 & 2 & $1.11 \pm 0.02$ & $23 \pm 2$ & $119 \pm 11$ & 8 & L6 & $\mathrm{L} 8 \pm 2.5$ & 14,2 & $1.386 \pm 0.080$ \\
\hline SDSS J142227.25+221557.1 & 5 & $0.07 \pm 0.03$ & $42 \pm 14$ & $14 \pm 6$ & 8 & $\ldots$ & $\mathrm{L} 6.5 \pm 2$ & 5 & $1.422 \pm 0.250$ \\
\hline 2MASS J14313097+1436539 & 9 & $0.43 \pm 0.04$ & 40: & 82: & 9 & L2 & $\mathrm{L} 3.5 \pm 1.5$ & 12,9 & $1.026 \pm 0.074$ \\
\hline 2MASS J14403186-1303263 & 1 & $0.45 \pm 0.05$ & 48: & $103 \pm 11$ & 1 & L1 & L1 pec (sl. blue) & 1,7 & $1.128 \pm 0.082$ \\
\hline 2MASS J17114559+4028578c & 7 & $0.25 \pm 0.02$ & $21.2 \pm 3.9$ & 25: & 8 & L5 & $\mathrm{L} 5 \pm 2$ & 7 & $1.205 \pm 0.075$ \\
\hline 2MASS J17210390+3344160 & 3 & $1.95 \pm 0.02$ & $16 \pm 1$ & $144 \pm 13$ & 8 & L3 & $\mathrm{L} 5 \pm 1$ & 3,10 & $1.136 \pm 0.030$ \\
\hline 2MASS J19495702+6222440 & 1 & $0.22 \pm 0.22$ & 65: & $68 \pm 68$ & 1 & L2 pec & L2 pec (sl. blue) & 1 & $1.609 \pm 0.167$ \\
\hline 2MASS J21513979+3402444 & 1 & $0.25 \pm 0.34$ & 27: & $32 \pm 44$ & 1 & $\ldots$ & L7 pec (sl. blue) & 1 & $1.721 \pm 0.187$ \\
\hline
\end{tabular}

Notes.

a SDSS J0805+4812: suspected binary comprised of mid-L and mid-T components; Burgasser et al. (2007).

b SIPS J0921-2104: also known as 2MASS J09211411-2104446.

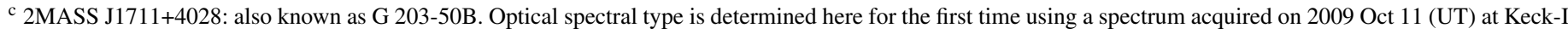
with LRIS (1200 s integration).

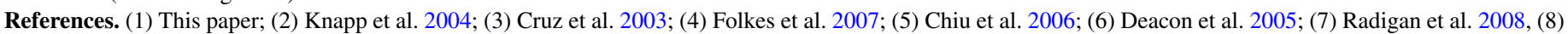

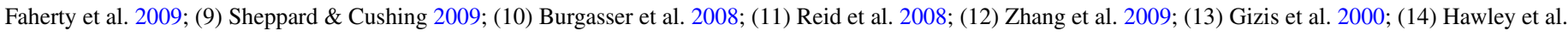
2002.

Table 11

New T Dwarfs

\begin{tabular}{|c|c|c|c|c|c|}
\hline $\begin{array}{c}\text { 2MASS Designation } \\
\text { (1) }\end{array}$ & $\begin{array}{c}\text { Opt. Sp. Ty. } \\
\text { (2) }\end{array}$ & $\begin{array}{l}\text { NIR Sp. Ty. } \\
\text { (3) }\end{array}$ & $\begin{array}{c}J-H \\
(4)\end{array}$ & $\begin{array}{c}H-K_{s} \\
\quad(5)\end{array}$ & $\begin{array}{c}J-K_{s} \\
(6)\end{array}$ \\
\hline 2MASS J11061191+2754215 & $\ldots$ & $\mathrm{T} 2.5$ & $0.674 \pm 0.069$ & $0.349 \pm 0.074$ & $1.023 \pm 0.066$ \\
\hline 2MASS J13243553+6358281 & $\ldots$ & $\mathrm{T} 2.5 \mathrm{pec}$ & $1.020 \pm 0.088$ & $0.518 \pm 0.082$ & $1.538 \pm 0.090$ \\
\hline 2MASS J14044948-3159330 & ear-T & $\mathrm{T} 3$ & $0.622 \pm 0.092$ & $0.417 \pm 0.116$ & $1.039 \pm 0.113$ \\
\hline 2MASS J15111466+0607431 ${ }^{\mathrm{a}}$ & $\ldots$ & $\mathrm{T} 2$ pec & $1.061 \pm 0.109$ & $0.411 \pm 0.125$ & $1.472 \pm 0.127$ \\
\hline 2MASS J21265916+7617440 & L7 & T0 pec & $0.750 \pm 0.050$ & $0.426 \pm 0.054$ & $1.176 \pm 0.050$ \\
\hline
\end{tabular}

Note. ${ }^{a}$ Discovered independently by Chiu et al. (2006).

Table 12

Other Interesting Discoveries

\begin{tabular}{|c|c|c|c|c|c|}
\hline $\begin{array}{c}\text { 2MASS Designation } \\
\text { (1) }\end{array}$ & $\begin{array}{c}\text { Opt. Sp. Ty. } \\
\text { (2) }\end{array}$ & $\begin{array}{c}\text { NIR Sp. Ty. } \\
\text { (3) }\end{array}$ & $\begin{array}{c}J-H \\
(4) \\
\end{array}$ & $\begin{array}{c}H-K_{S} \\
(5)\end{array}$ & $\begin{array}{c}J-K_{s} \\
(6)\end{array}$ \\
\hline 2MASS J04205430-7327392 & $\ldots$ & $\ldots$ & $1.354 \pm 0.034$ & $1.602 \pm 0.032$ & $2.956 \pm 0.032$ \\
\hline 2MASS J09073735-1457036 & $\ldots$ & $\ldots$ & $0.132 \pm 0.115$ & $0.343 \pm 0.183$ & $0.475 \pm 0.156$ \\
\hline 2MASS J16002647-2456424 & $\ldots$ & M7.5 pec & $0.600 \pm 0.084$ & $0.301 \pm 0.103$ & $0.901 \pm 0.084$ \\
\hline 2MASS J18284076+1229207 & M8 sl pec & M7.5 pec & $0.471 \pm 0.066$ & $0.566 \pm 0.082$ & $1.037 \pm 0.078$ \\
\hline 2MASS J21403907+3655563 & $\ldots$ & M8 pec & $0.539 \pm 0.103$ & $0.396 \pm 0.132$ & $0.935 \pm 0.127$ \\
\hline
\end{tabular}

look for any peculiarities with respect to the standard sequence. Objects falling midway between integral classes (such as L5 and L6) were assigned the half-class in between (in this case, L5.5). Objects showing notable peculiarities were given a suffix of "pec." If the peculiarities were determined to be only slight, the suffix "sl. pec" was used instead. For a few of these, the underlying physical reason appears to be low gravity, so those are also labeled with either a "(low-g)" or a $\beta / \gamma / \delta$ (Cruz et al. 2009, J. D. Kirkpatrick et al. 2010, in preparation) suffix as further discussed in Section 6.1. Other peculiar spectra were later determined to be low-metallicity objects and were reclassified against a suite of subdwarf standards. These objects, given a prefix of "sd," are described further in Section 6.3.

\subsection{Near-infrared Types: Building a Standard Sequence}

Spectral classification in the near-infrared should be accomplished the same way as in the optical. Surprisingly, however, 
Table 13

Measured Velocities and Distance Estimates

\begin{tabular}{|c|c|c|c|c|c|c|c|}
\hline 2MASS Designation & Source $^{\mathrm{a}}$ & Sp. Ty. & $\begin{array}{l}\text { Obs. Date } \\
\text { (UT) } \\
\text { (4) }\end{array}$ & $\begin{array}{c}v_{\mathrm{rad}} \\
\left(\mathrm{km} \mathrm{s}^{-1}\right) \\
(5)\end{array}$ & $\begin{array}{l}\text { Dist. Est. } \\
\text { (pc) } \\
(6)\end{array}$ & $\begin{array}{c}v_{\tan } \\
\left(\mathrm{km} \mathrm{s}^{-1}\right) \\
(7)\end{array}$ & $\begin{array}{c}\text { Tot. Space Vel. } \\
\left(\mathrm{km} \mathrm{s}^{-1}\right) \\
(8)\end{array}$ \\
\hline \multicolumn{8}{|l|}{ Low-gravity Objects } \\
\hline 2MASS J00040288-6410358 & optical & $\mathrm{L} 1 \gamma$ & 2006 Nov 21 & $-57 \pm 50$ & 57 & $92 \pm 54$ & $108 \pm 74$ \\
\hline 2MASS J02340093-6442068 & optical & $\mathrm{L} 0 \gamma$ & 2005 Dec 4 & $-46 \pm 50$ & 45 & $133 \pm 109$ & $140 \pm 120$ \\
\hline 2MASS J03032042-7312300 & optical & $\mathrm{L} 2 \gamma$ & 2006 Nov 21 & $-21 \pm 50$ & 59 & $382 \pm 256$ & $383 \pm 261$ \\
\hline \multirow[t]{2}{*}{ 2MASS J04062677-3812102 } & optical & $\mathrm{L} 0 \gamma$ & 2009 Oct 11 & $-76 \pm 50$ & 90 & $103 \pm 120$ & $128 \pm 130$ \\
\hline & optical & $\mathrm{L} 0 \gamma$ & 2009 Oct 12 & $-67 \pm 50$ & 90 & $103 \pm 120$ & $123 \pm 130$ \\
\hline 2MASS J05341594-0631397 & optical & M8 $\gamma$ & 2009 Oct 11 & $-93 \pm 50$ & 223 & $234 \pm 138$ & $251 \pm 147$ \\
\hline 2MASS J15575011-2952431 & optical & M9 $\delta$ & 2008 Aug 12 & $4 \pm 50$ & 140 & $173 \pm 73$ & $173 \pm 89$ \\
\hline \multicolumn{8}{|l|}{ Red L Dwarfs } \\
\hline 2MASS J13313310+3407583 & optical & LO & 2009 Feb 17 & $-151 \pm 50$ & 14 & $29 \pm 4$ & $154 \pm 50$ \\
\hline 2MASS J18212815+1414010 & optical & $\mathrm{L} 4.5$ & 2007 Aug 20 & $243 \pm 50$ & 10 & $11 \pm 5$ & $243 \pm 50$ \\
\hline \multirow[t]{3}{*}{ 2MASS J21481633+4003594 } & near-IR & L6/7 pec & $2005 \operatorname{Dec} 9$ & $55 \pm 50$ & 10 & $66 \pm 12$ & $86 \pm 52$ \\
\hline & near-IR & L6/7 pec & 2006 Aug 4 & $-13 \pm 50$ & 10 & $66 \pm 12$ & $67 \pm 52$ \\
\hline & optical & L6 & 2007 Aug 21 & $183 \pm 50$ & 10 & $66 \pm 12$ & $195 \pm 52$ \\
\hline 2MASS J23174712-4838501 & optical & L4 pec & 2007 Aug 19 & $155 \pm 50$ & 24 & $42 \pm 19$ & $161 \pm 54$ \\
\hline 2MASS J23512200+3010540 & optical & $\mathrm{L} 5.5$ & 2008 Oct 5 & $177 \pm 50$ & 23 & $31 \pm 8$ & $180 \pm 51$ \\
\hline \multicolumn{8}{|l|}{ Late-M and L Subdwarfs } \\
\hline 2MASS J04470652-1946392 & optical & sdM7.5 & 2007 Aug 20 & $110 \pm 50$ & 102 & $325 \pm 39$ & $344 \pm 63$ \\
\hline 2MASS J06453153-6646120 & optical & sdL8 & 2008 Feb 23 & $126 \pm 50$ & 16 & $120 \pm 6$ & $174 \pm 50$ \\
\hline \multirow[t]{2}{*}{ 2MASS J11582077+0435014 } & near-IR & sdL7 & $2005 \operatorname{Dec} 9$ & $81 \pm 50$ & 16 & $94 \pm 18$ & $124 \pm 53$ \\
\hline & optical & sdL7 & 2009 Feb 17 & $130 \pm 50$ & 16 & $94 \pm 18$ & $161 \pm 53$ \\
\hline \multirow[t]{2}{*}{ 2MASS J17561080+2815238 } & near-IR & sdL1 & 2006 Aug 4 & $-176 \pm 50$ & 35 & $147 \pm 22$ & $229 \pm 54$ \\
\hline & optical & sdL1 & 2007 Aug 21 & $-182 \pm 50$ & 35 & $147 \pm 22$ & $234 \pm 54$ \\
\hline \multicolumn{8}{|l|}{ Blue L Dwarfs } \\
\hline 2MASS J00150206+2959323 & Optical & L7 & 2008 Aug 13 & $-71 \pm 50$ & 21 & $50 \pm 9$ & $87 \pm 51$ \\
\hline 2MASS J10461875+4441149 & Near-IR & L4(L5pec) & $2005 \operatorname{Dec} 9$ & $105 \pm 50$ & 24 & $31 \pm 8$ & $109 \pm 51$ \\
\hline 2MASS J11181292-0856106 & Optical & L6 & 2006 May 8 & $250 \pm 50$ & 21 & $59 \pm 14$ & $257 \pm 52$ \\
\hline 2MASS J13023811+5650212 & Optical & L2 & 2009 Feb 17 & $117 \pm 50$ & 65 & $102 \pm 84$ & $155 \pm 97$ \\
\hline 2MASS J14403186-1303263 & Optical & L1 & 2008 Aug 12 & $-40 \pm 50$ & 48 & $103 \pm 11$ & $110 \pm 51$ \\
\hline 2MASS J19495702+6222440 & Optical & L2 pec & 2009 Oct 12 & $104 \pm 50$ & 65 & $68 \pm 68$ & $124 \pm 84$ \\
\hline
\end{tabular}

Notes. See Sections 6.1 and 6.3 for an object-by-object discussion of the distance estimates for the low-gravity objects and subdwarfs. Distances to the red and blue L dwarfs have been estimated with the Looper et al. (2008) relations and use the listed spectral type and measured 2MASS $J$ magnitude.

a Type of spectrum, as given in Table 2, from which the listed spectral type and radial velocity were measured.

a set of near-infrared $\mathrm{M}$ and $\mathrm{L}$ dwarf anchors has never been established although those for $\mathrm{T}$ dwarfs have been (Burgasser et al. 2006). Previous near-infrared classifications, such as those in Reid et al. (2001), Geballe et al. (2002), and McLean et al. (2003), have relied on comparing a target object's spectral ratios with those same ratios derived for an object of known optical type. These optically classified comparison objects, however, might not necessarily create a smooth near-infrared spectral sequence across all of the $J, H, K$ spectral windows (1.0-2.5 $\mu \mathrm{m})$ since that was not a requirement. Now that a very large number of spectra have been acquired over these wavelengths (e.g., the suite of SpeX prism data available at http://www.browndwarfs.org/spexprism, as well as the set of SpeX spectra acquired here), it is now possible to create a grid of spectra whose near-infrared features change smoothly and monotonically as a function of type. This spectral morphological sequence was built to satisfy the following properties.

1. The near-infrared spectral standards should have nearinfrared types identical or very close to the types assigned to them in the optical. In this way it can be assured that a random $\mathrm{M}$ or $\mathrm{L}$ dwarf will, on average, have identical or very similar optical and near-infrared classifications. Primary standards from the optical classification sequence have been reused here if they satisfy the other criteria below.
2. The near-infrared color of each primary standard should be as close as possible to the median $J-K_{s}$ color of other objects with that same optical classification. Median colors as a function of optical type are given in Table 3 of Kirkpatrick et al. (1994) for early- to mid-M dwarfs and Figure 14 of Kirkpatrick et al. (2008) for late-M and L dwarfs. The goal of this criterion is to assure that each nearinfrared standard describes the average color (and hence, presumably, overall morphological shape over the $J, H$, and $K$ bandpasses) of objects in that class.

3. The resulting spectral sequence should be smoothly continuous in morphology. That is, each primary standard should be analogous to a frame in a movie, where each frame is only subtly different from the frames (standards) on either side.

In cases where multiple objects at a single class met the above three requirements, objects were given preference if they were relatively bright or if they fell within $30^{\circ}$ of the celestial equator. The resulting M0-L9 near-infrared primary standards are listed in Table 4, and the full M0-T8 near-infrared spectral sequence is illustrated in Figures 9-11. It should be noted that no suitable near-infrared L1 standard was found with an optical type of L1, so an object of optical type L1.5 was used instead. Likewise, at near-infrared type L7 an object of optical type L6 was used. 


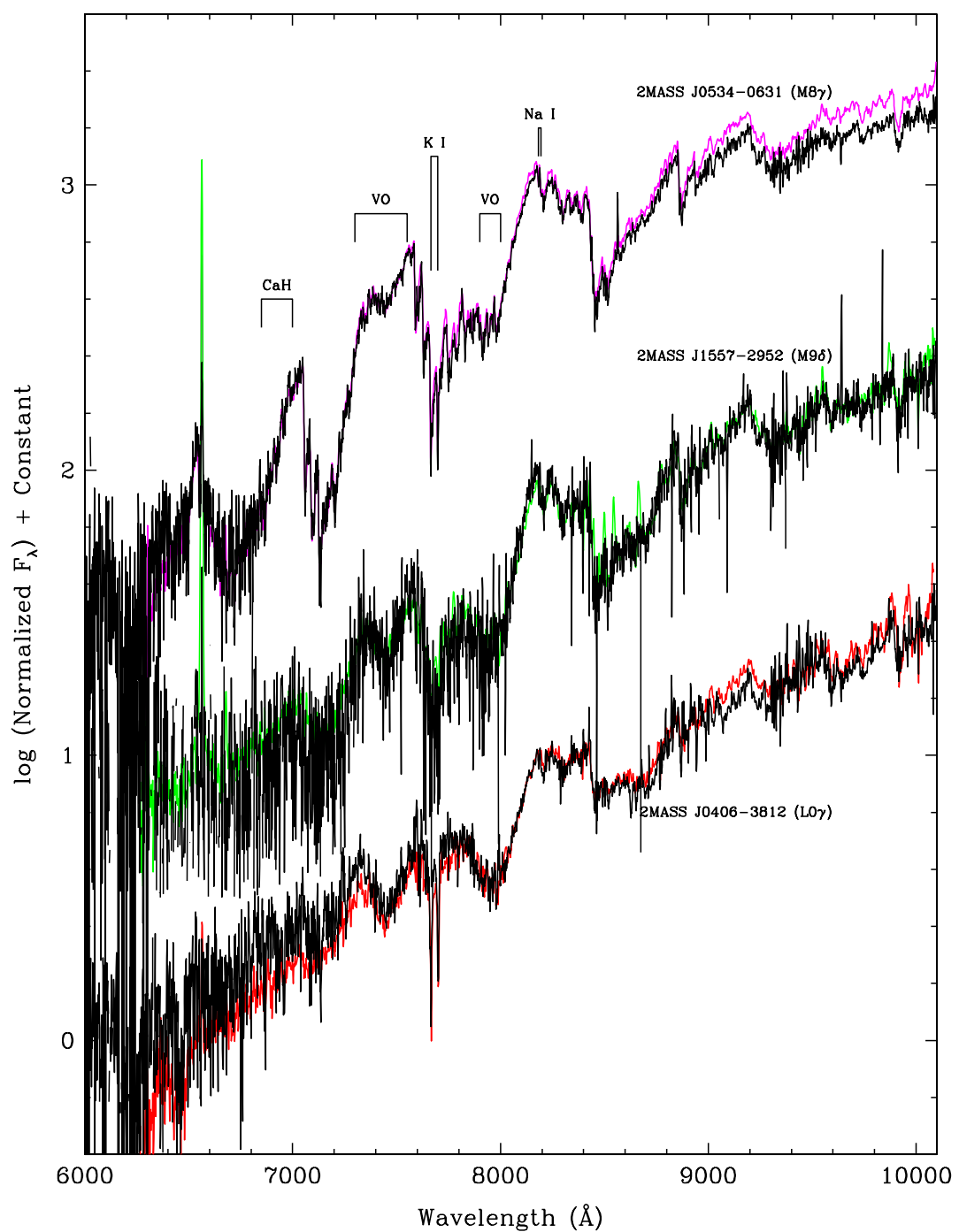

Figure 12. Optical spectra of low-gravity dwarf discoveries (black lines) with late-M to early-L types. Overplotted for comparison are the low-gravity M8 dwarf 2MASS J1207-3932 (magenta), the low-gravity M9 dwarf KPNO-Tau 12 (green), and the L0 $\gamma$ dwarf 2MASS J0141-4633 (red). Spectra are normalized to one at $8250 \AA$ and integer offsets added when needed to separate the spectra vertically.

(A color version of this figure is available in the online journal.)

The optical spectral sequence does not include an L9 class-in fact, there is a rather large jump in morphology between known optical L8 dwarfs and the optical T0 standard (Kirkpatrick et al. 2008) - so an optical L8 that bridged the morphological gap between the near-infrared L8 standard and the near-infrared T0 standard of Burgasser et al. (2006) was used in its place.

With this suite of standards in hand, near-infrared spectral classifications for the SpeX, NIRSPEC, and OSIRIS data were performed as follows. Each target spectrum was normalized to one at $1.28 \mu \mathrm{m}$ and compared to the near-infrared spectral standards from Figures 9-11 normalized the same way. The core near-infrared type was determined only from the 0.9-1.4 $\mu \mathrm{m}$ portion. With that best match in hand, the corresponding goodness of fit to the same spectral standard from 1.4 to $2.5 \mu \mathrm{m}$ was judged. In most cases the same spectral standard also provided the best fit in this region. In other cases, the target spectrum was notably much bluer or redder so the fit across the $H$ and $K$ windows was very poor despite the excellent fit in the $J$ window. These objects were given suffixes of "blue" or "red" (or for ones only slightly discrepant, "sl. blue" or "sl. red") to denote the slope of the spectrum relative to the standard. Other spectra simply had no good match even in the $0.9-1.4 \mu \mathrm{m}$ window, so those were given a suffix of "pec." Some of these were deemed to be either low gravity ("low-g") or low metallicity (prefix of "sd"), as discussed further in the sections that follow.

Interesting objects and comparable, previously published objects of similar type are discussed below and tabulated in Tables 5-12. For our noteworthy discoveries having high-quality spectra with $R \geqslant 1000$, we also derive radial velocities and then derive distance estimates and tangential velocities to compute total space motions in Table 13. Radial velocities are derived using standard cross-correlation to objects of known radial velocity and similar spectral type. Because of the relatively lowresolution of our spectra, these radial velocity measurements typically have large uncertainties, which we conservatively overestimate to be $\sim 50 \mathrm{~km} \mathrm{~s}^{-1}$.

\section{OBJECTS WORTHY OF SPECIAL NOTE}

\subsection{Young (Low-gravity) Objects}

Some of the peculiar late-M and L dwarf spectra were recognized as being unusual because they have much lower 


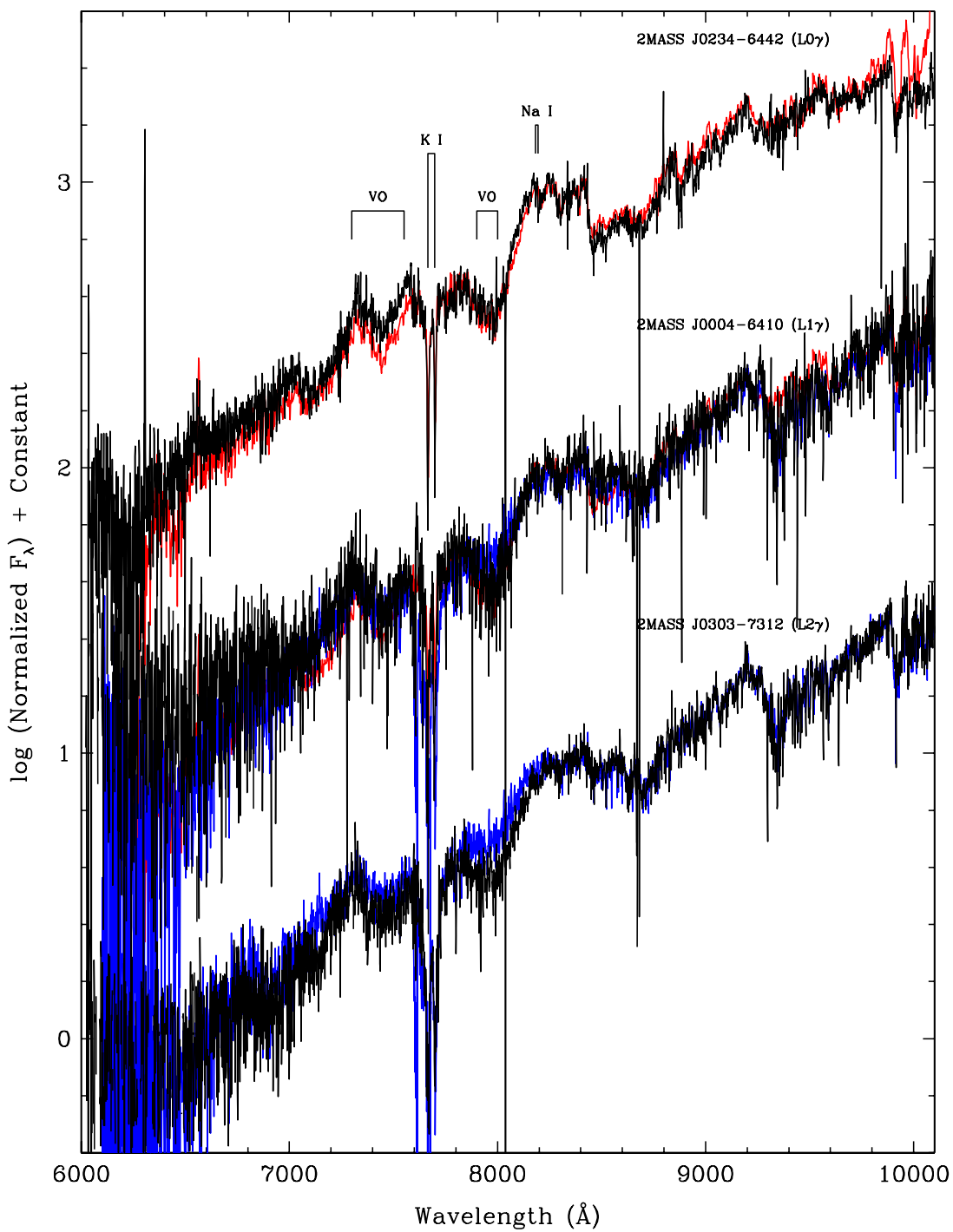

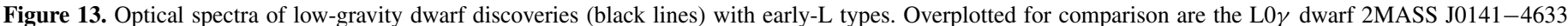

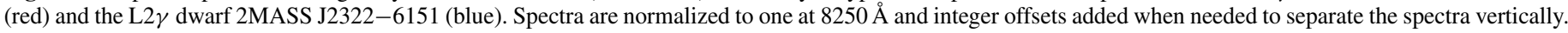
(A color version of this figure is available in the online journal.)

gravity than normal field dwarfs. Low gravity in these objects is the observational by-product of youth. These objects are less massive than an older dwarf of the same temperature (i.e., at the same mass, younger objects have had less time to cool) and have more distended atmospheres because they have not yet completed their gravitational contraction. Both effects contribute to the lowering of the gravity relative to a collapsed, more massive object of similar temperature.

The first late-M dwarfs exhibiting the telltale signs of low gravity were identified over a decade ago in young clusters such as the Pleiades (Steele \& Jameson 1995), the $\rho$ Ophiuchi star formation region (Luhman et al. 1997), and the Taurus-Auriga complex (Luhman et al. 1998). Many other low-gravity late$\mathrm{M}$ dwarfs are now recognized both in clusters as well as in loose, young associations. The first $\mathrm{L}$ dwarf recognized as young through its low-gravity features was the field L0 dwarf 2MASS J01415823-4633574 (Kirkpatrick et al. 2006). This object, predicted to be a member of either the 12 Myr old $\beta$ Pictoris moving group or the $30 \mathrm{Myr}$ old Tucana-Horologium association from its sky location and low-gravity signatures, now has a proper motion measurement by Faherty et al. (2009) that is statistically consistent with membership in Tucana-Horologium. This leads to a predicted distance of $44 \pm 8 \mathrm{pc}$ and a radial velocity of $\sim+8 \mathrm{~km} \mathrm{~s}^{-1}$ (E. Mamajek 2009, private communication), both of which await verification. Many other low-gravity field $M$ and $\mathrm{L}$ dwarfs have now been recognized because of their low-gravity signatures (Kirkpatrick et al. 2008; Cruz et al. 2009), and these are now the targets of dedicated astrometric and radial velocity programs that will establish the $U V W$ space motions needed to confirm membership in young moving groups.

It has been shown that $7.6 \% \pm 1.6 \%$ of field $\mathrm{L}$ dwarfs exhibit signs of lower gravity and are presumably younger than $100 \mathrm{Myr}$, in agreement with theoretical expectations (Kirkpatrick et al. 2008). However, proper motion studies are biased toward objects with halo kinematics, so one would not expect a significant contribution by young objects. Indeed, all six of our young discoveries below have measured proper motions that are within $1 \sigma$ or $2 \sigma$ of zero and were primarily targeted for follow-up due to their red colors.

We identify six objects, listed in Table 5, that show the hallmarks of lower gravity. Optical spectra for all of these are shown in Figures 12 and 13, and near-infrared spectra 


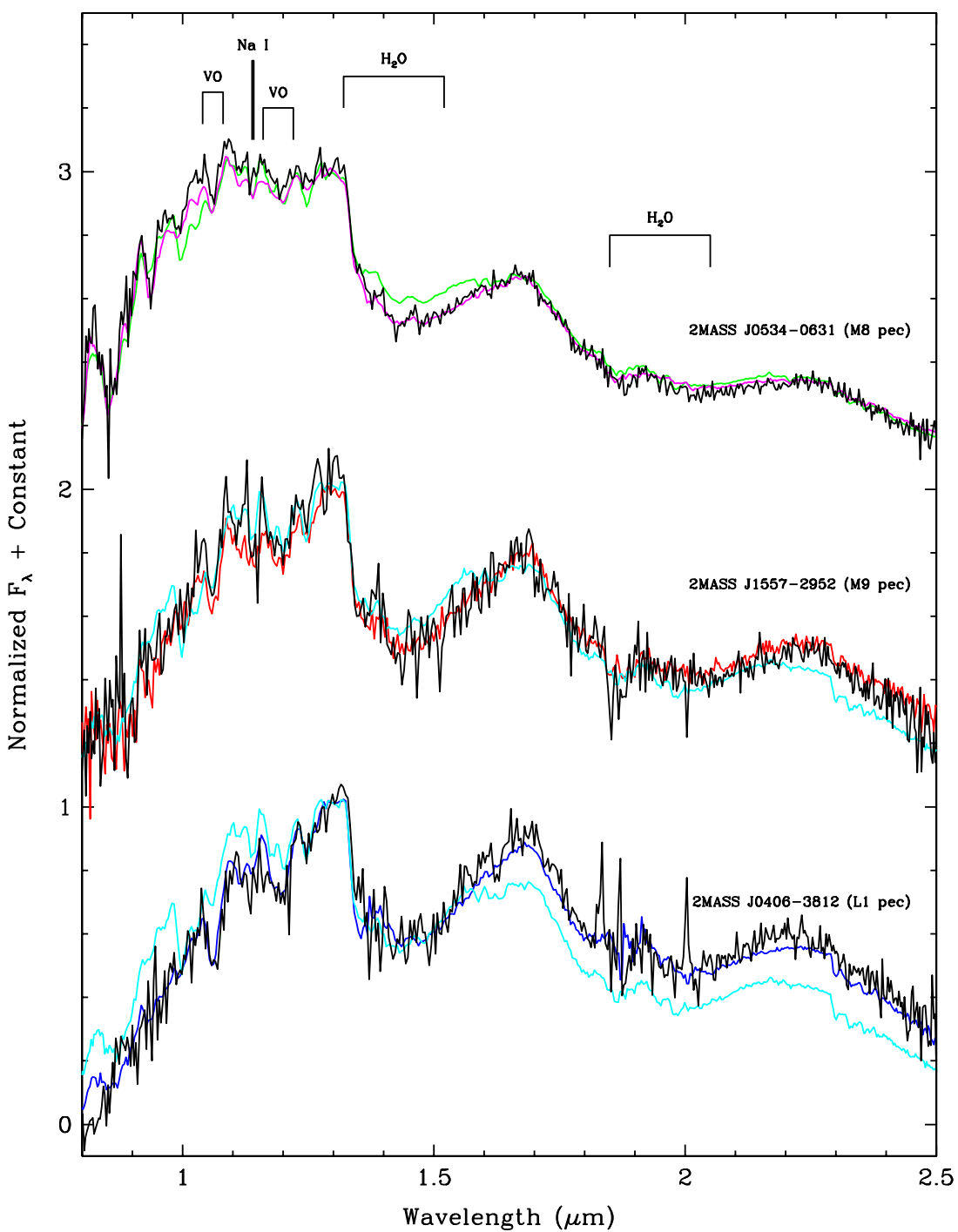

Figure 14. Near-infrared spectra of low-gravity discoveries (black lines). Overplotted for comparison are the standard M8 dwarf vB 10 (green), the low-gravity M8 dwarf 2MASS J1207-3932 (magenta), the low-gravity M9 dwarf KPNO-Tau 12 (red), the standard L1 dwarf 2MASS J2130-0845 (cyan), and the low-gravity L1 dwarf 2MASS J0141-4633 (blue). Spectra are normalized to one at $1.28 \mu \mathrm{m}$ and integer offsets added when needed to separate the spectra vertically.

(A color version of this figure is available in the online journal.)

of three are shown in Figure 14. As has been true in other studies (Kirkpatrick et al. 2008; Cruz et al. 2009), these young field objects tend to be found in the southern sky where, not coincidentally, most of the young associations are concentrated. For optical spectral types we use the Greek suffix designations of Cruz et al. (2009) in which $\alpha$ (usually dropped for convenience) is used to denote objects of normal gravity, $\beta$ for intermediate gravity, and $\gamma$ for low gravity. Objects of extremely low gravity are further denoted by $\delta$, as originally outlined in Kirkpatrick (2005) and Kirkpatrick et al. (2006).

2MASS J00040288-6410358. The optical spectrum of this object (Figure 13) falls intermediate between that of 2MASS J0141-4633 and 2MASS J23225299-6151275, which are classified as $\mathrm{L} 0 \gamma$ and $\mathrm{L} 2 \gamma$, respectively, on the scheme of Cruz et al. (2009). We therefore assign 2MASS J0004-6410 an optical spectral type of $\mathrm{L} 1 \gamma$. Assuming its absolute $J$ magnitude is identical to that of a normal L1 dwarf, then the magnitude estimates from Table 3 of Looper et al. (2008) can be used to predict a distance of $\sim 57 \mathrm{pc}$ for this object. The spectrophotometric distance estimate, sky location, and similarity to 2MASS J0141-4633 suggest that 2MASS J0004-6410 may be a mem- ber of the $\sim 50 \mathrm{pc}$ distant, 30 Myr old Tucana-Horologium association (Torres et al. 2008).

2MASS J02340093-6442068. The optical spectrum of this object (Figure 13) is a good match to that of 2MASS J0141-4633. On the classification scheme of Cruz et al. (2009) this gives 2MASS J0234-6442 an optical spectral type of L0 $\gamma$. Assuming its absolute $J$ magnitude is identical to that of a normal L0 dwarf, then the magnitude estimates from Table 3 of Looper et al. (2008) can be used to predict a distance of $\sim 45 \mathrm{pc}$ for this object. The spectrophotometric distance estimate, sky location, and similarity to 2MASS J0141-4633 suggest that 2MASS J0234-6442 may also be a member of the Tucana-Horologium association.

2MASS J03032042-7312300. The optical spectrum of this object (Figure 13) falls intermediate between 2MASS J00452143+1634446 (L2 $\beta$ ) and 2MASS J2322-6151 (L2 $\gamma$ ). Because 2MASS J0303-7312 has very weak NaI at 8183$8195 \AA$, we believe that a classification of $\mathrm{L} 2 \gamma$ is the more appropriate one. Assuming its absolute $J$ magnitude is identical to that of a normal L2 dwarf, the magnitude estimates from Table 3 of Looper et al. (2008) can be used to predict a distance 


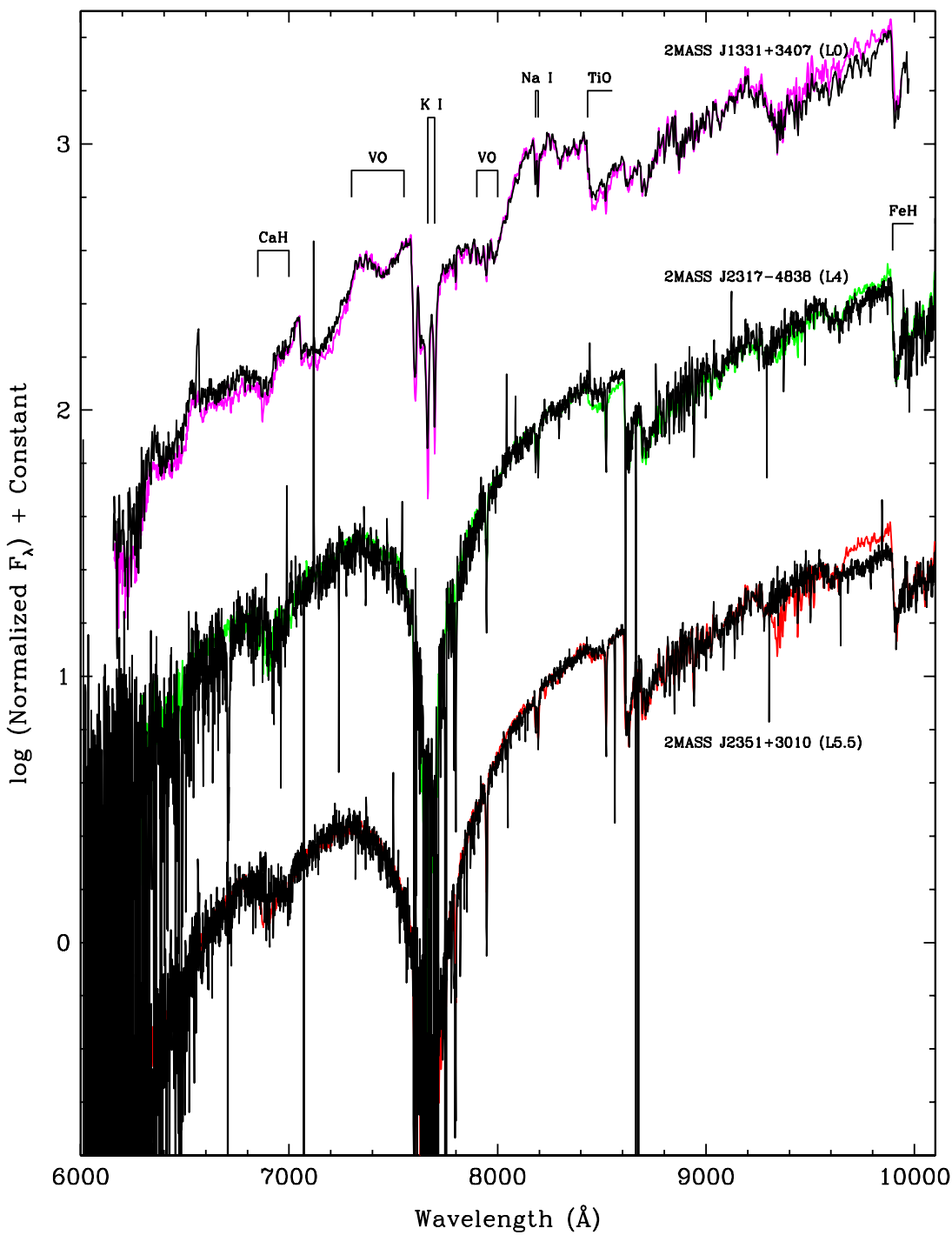

Figure 15. Optical spectra of three red L dwarf discoveries (black lines). Overplotted for comparison are optical standards: the L0 dwarf 2MASS J0345+2540 (magenta), L4 dwarf 2MASS J1155+2307 (green), and L5 dwarf DENIS J1228-1547 (red). Spectra are normalized to one at $8250 \AA ̊$ and integer offsets added when needed to separate the spectra vertically.

(A color version of this figure is available in the online journal.)

of $\sim 59 \mathrm{pc}$ for this object. As with the two objects noted above, the spectrophotometric distance estimate, sky location, and $\gamma$ suffix suggest that 2MASS J0303-7312 may also be a member of the Tucana-Horologium association.

2MASS J04062677-3812102. The optical spectrum of this object (Figure 12) is a good match to that of 2MASS J0141-4633, giving 2MASS J0406-3812 an optical type of L $0 \gamma$. Its near-infrared spectrum (Figure 14) roughly matches a type of L1 but has some peculiarities. The spectrum is redder than the $\mathrm{L} 1$ standard, the $H$-band peak is much more triangular, and there are much stronger $\mathrm{VO}$ bands at 1.06 and $1.18 \mu \mathrm{m}$ than normally seen in an L1. These discrepancies mimic those seen in the near-infrared spectrum of 2MASS J0141-4633 (Kirkpatrick et al. 2006), which provides an excellent match to the overall shape of 2MASS J0406-3812. Its L1 near-infrared spectral type and $J$-band magnitude suggest a distance of $90 \mathrm{pc}$, and the match to 2MASS J0141-4633 suggests an age of very roughly $30 \mathrm{Myr}$ (assuming 2MASS J0141-4633 itself is a member of the Tucana-Horologium association). Given these clues as well as its sky location, 2MASS J0406-3812 may therefore be a member of the 90 pc distant, 30 Myr old Columba association (Torres et al. 2008).

2MASS J05341594-0631397. The optical spectrum of this object (Figure 12) is a good match to the M8 $\gamma$ dwarf 2MASS $\mathrm{J} 12073346-3932539 \mathrm{~A}$, which is a member of the $8 \mathrm{Myr}$ old TW Hydrae Association. (The gravity classification suffix $\gamma$ is given to $\mathrm{M}$ dwarfs with $\log ($ age $(\mathrm{yr})) \approx 7$; J. D. Kirkpatrick et al. 2010, in preparation) The near-infrared spectrum (Figure 14) shows peculiarities when compared to a standard late-M dwarf but is again almost identical to the low-gravity $\mathrm{M}$ dwarf 2MASS J1207-3932A. Using the trigonometric parallax ( $\pi=$ 0'01851 \pm 0 '.00103; Gizis et al. 2007) and $J$-band magnitude (12.995 mag) of 2MASS J1207-3932A as a guide, we estimate that 2MASS J0534-0631 is roughly 223 pc distant. This distance estimate places it further away than any of the young associations studied by Zuckerman \& Song (2004) or Torres et al. (2008).

2MASS J15575011-2952431. The optical spectrum of this object shows the telltale signs of a low-gravity late-M dwarf (Figure 12), namely weaker $\mathrm{TiO}$ bands and alkali lines together 


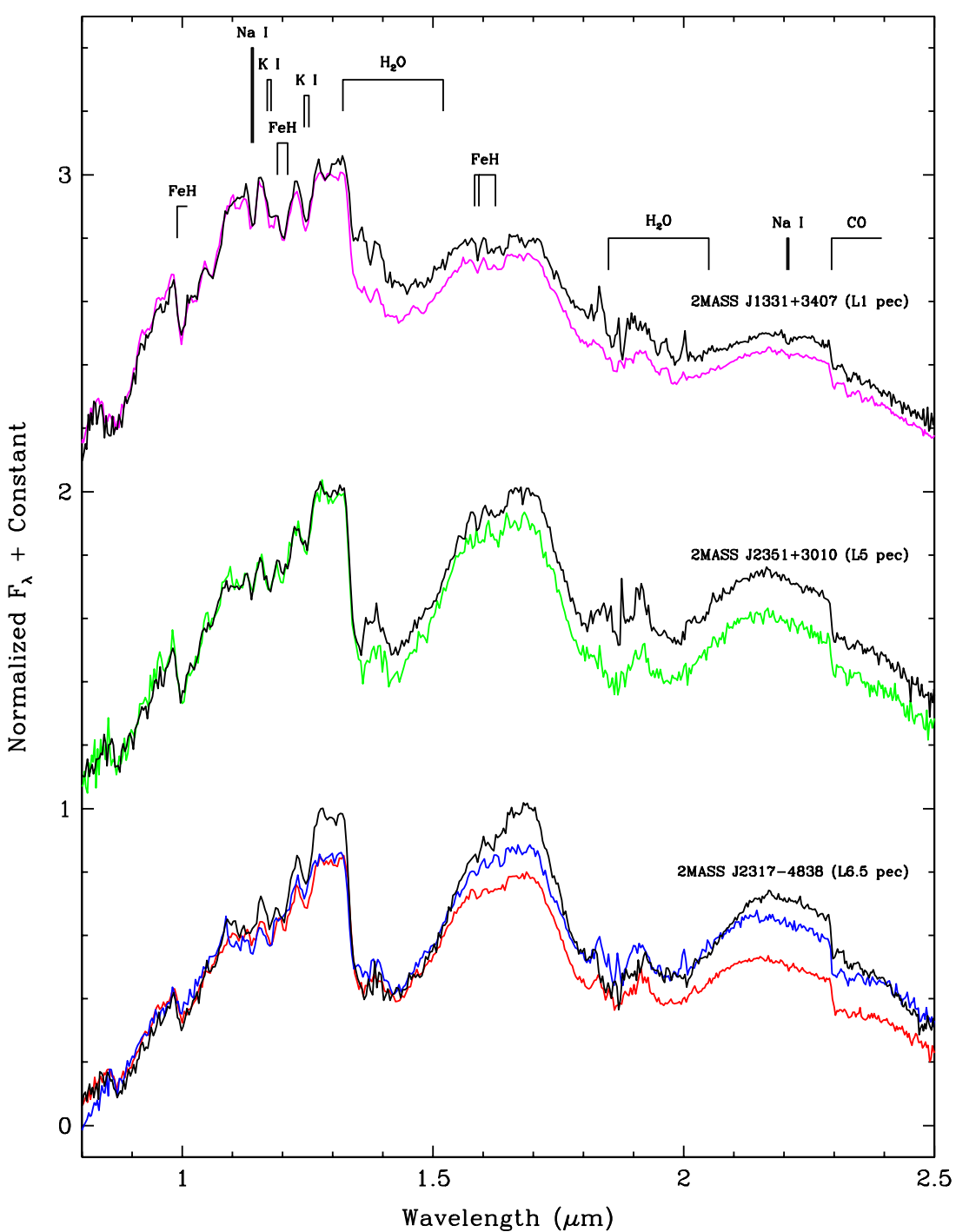

Figure 16. Near-infrared spectra of three red L dwarf discoveries (black lines). Overplotted for comparison are near-infrared standards: the L1 dwarf $2 \mathrm{MASS}$ J2130-0845 (magenta), L5 dwarf 2MASS J0835+1953 (green), L6 dwarf 2MASS J1010-0406 (red), and L7 dwarf 2MASS J0103+1935 (blue). Spectra are generally normalized to one at $1.28 \mu \mathrm{m}$ and integer offsets added when needed to separate the spectra vertically. The spectra of 2MASS J1010-0406 and 2MASS J0103+1935 have been scaled at $1.0 \mu \mathrm{m}$ to match the flux level of 2MASS J2317-4838 to maximize the agreement among all three spectra at the shortest wavelengths shown.

(A color version of this figure is available in the online journal.)

with stronger VO bands. The overall spectral morphology is very similar to that of the $1 \mathrm{Myr}$ old Taurus member KPNO-Tau 12 (aka 2MASS J04190126+2802487). A tentative core type of M9 is therefore assigned, and a suffix of $\delta$ is given because that is the suffix given to M dwarfs like KPNO-Tau 12 with $\log (\operatorname{age}(\mathrm{yr})) \approx 6$ (J. D. Kirkpatrick et al. 2010, in preparation) The near-infrared spectrum also shows the hallmarks of lower gravity (Figure 14) - a triangular-shaped $H$-band peak and a strong VO band at $1.08 \mu \mathrm{m}-$ which are again similar to those features seen in KPNO-Tau 12. Given that 2MASS J1557-2952 and KPNO-Tau 12 have similar optical classifications and $J$-band magnitudes (2MASS measures of 16.316 and 16.305 mag, respectively), we can assume they fall at roughly the same distance, which for KPNO-Tau 12 is assumed to be that of the Taurus Molecular Cloud (140 pc; Luhman et al. 2006). Given the sky location, estimated distance, and young implied age of 2MASS J1557-2952, it may be a member of the broad Scorpius-Centaurus complex.

Radial velocities, tangential velocities, and total space motions for these six low-gravity objects are given in Table 13. The measured proper motions for these objects, with the exception of 2MASS J1557-2952, are within $2 \sigma$ of zero motion, and an accurate derivation of the tangential velocity is further complicated by the fact that these objects are more distant than most other objects listed in Table 13. The radial velocity measurements, which are independent of distance, are also within $2 \sigma$ of zero motion. Despite the large uncertainties, the lack of high radial velocities in this list is nonetheless consistent with our interpretation of these objects as young.

\subsection{Unusually Red L Dwarfs}

The first unusually red L dwarf, 2MASS J22443167+2043433 (Dahn et al. 2002), was discovered during the search for red active galactic nuclei with 2MASS. Its 2MASS color of $J-K_{s}=$ $2.45 \pm 0.16$ is far redder than the mean $J-K_{s}$ color of other L6.5 dwarfs (see Figure 14 of Kirkpatrick et al. 2008), and its near-infrared spectrum is markedly odd compared to most other late-L dwarfs (McLean et al. 2003). We now believe that the redness of 2MASS J2244+2043 is caused by low gravity (Kirkpatrick et al. 2008) and low-gravity L dwarfs can become 


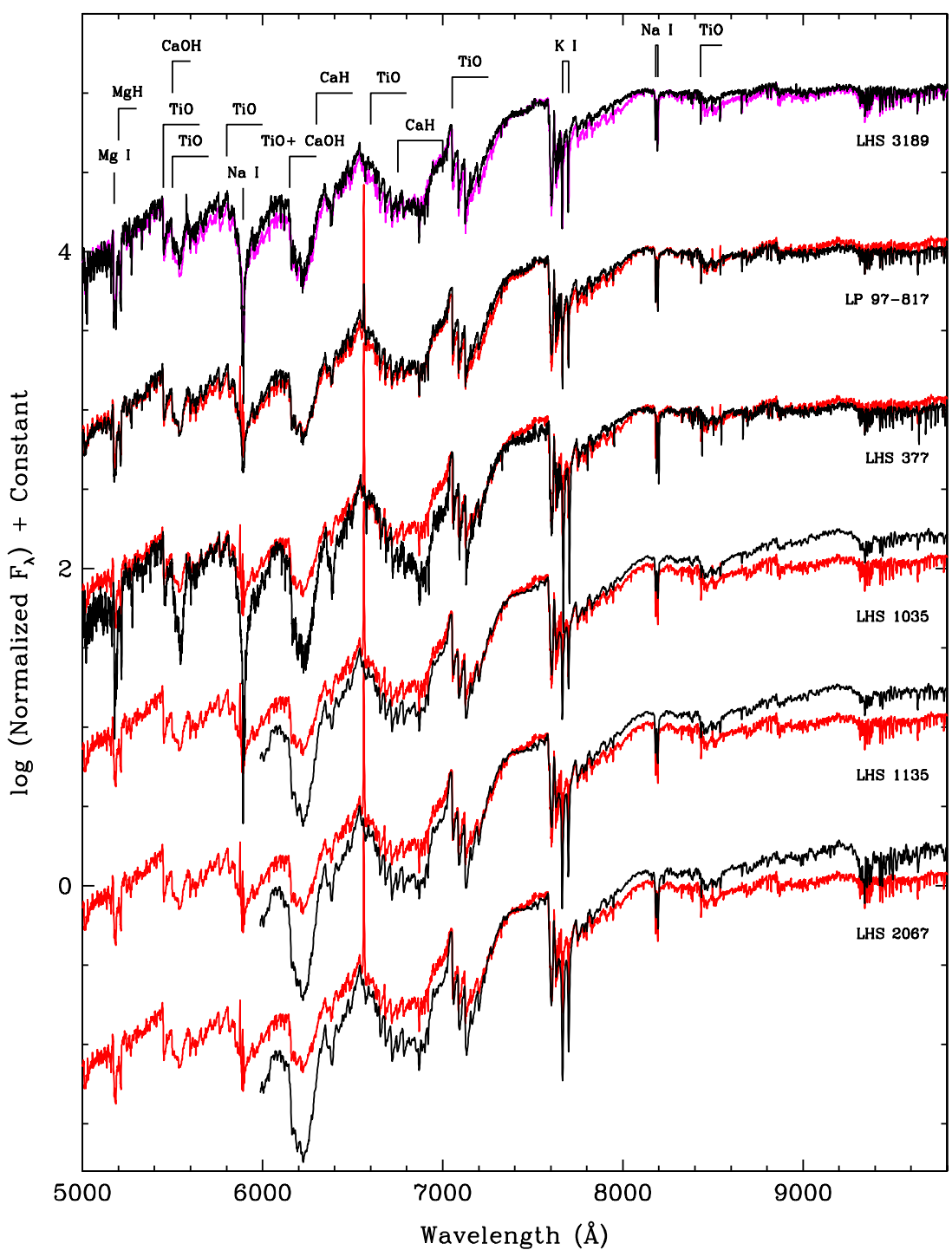

Figure 17. Optical spectra from Keck/DEIMOS (top three objects, in black) or Gemini-North/GMOS (bottom three objects, in black) compared to normal dwarfs of type M4.5 (LHS 3001, magenta) or M5 (Gliese 51, red) taken with Keck/DEIMOS. Spectra are renormalized so that the target and comparison spectra overlap either at 7500 or $8250 \AA$. Integer offsets have been added when needed to separate the spectra vertically.

(A color version of this figure is available in the online journal.)

even redder than this, as evinced by the TW Hydrae association member 2MASS J12073346-3932539B, a late-L dwarf with $J-K \sim 3.05$ (Gizis et al. 2007).

As mentioned in the above section, a cause has been proposed (Kirkpatrick et al. 2006) and a classification system developed (Cruz et al. 2009) for these low-gravity, red L dwarfs. Our proper motion survey has uncovered a few other red L dwarfs, however, whose peculiarities cannot as easily be attributed to low gravity, and for these we assign a suffix of "pec" to indicate their peculiar morphology in the near-infrared. The oddest of these, 2MASS J21481633+4003594, has been discussed at length by Looper et al. (2008) along with another bright example, 2MASS $\mathrm{J} 18212815+1414010$, both of which were discovered during our proper motion survey. We discuss three other red L dwarf discoveries below:

2MASS J13313310+3407583. In the optical (Figure 15) this object most closely resembles an L0 dwarf. In the near-infrared (Figure 16) it most closely resembles an L1 at $J$ band. If the flux of the 2MASS J1331+3407 spectrum is normalized to the $J$ band of the L1 near-IR standard, the $H$ - and $K$-band portions of the 2MASS J1331+3407 spectrum have too much flux relative to the standard (hence the "red" designation). This redness is also obvious in the shallow depth of the $\mathrm{H}_{2} \mathrm{O}$ bands between the $J$ - and $H$-band peaks and again between the $\mathrm{H}$ and $\mathrm{K}$ peaks. The 2MASS color of $J-K_{s}=1.448 \pm 0.035 \mathrm{mag}$ is $\sim 0.2 \mathrm{mag}$ redder than the median color of optically defined L0 dwarfs in Figure 14 of Kirkpatrick et al. (2008).

2MASS J23174712-4838501. This object most closely resembles an L4 in the optical (Figure 15), the only major deviation being that its $8432 \AA \mathrm{TiO}$ band is weaker than the L4 standard and more like that of an L5 or L6 dwarf. In the nearinfrared, the spectrum continues to match that of an L6 up to $\sim 1.1 \mu \mathrm{m}$. At longer wavelengths - even in the longer half of the $J$ bandpass - there is excess flux relative to both the L6 and L7 near-infrared standards. The 2MASS color of $J-K_{s}=$ $1.969 \pm 0.048$ mag is $\sim 0.2$ mag redder than the median color of optically defined L4 dwarfs in Figure 14 of Kirkpatrick et al. (2008).

2MASS J23512200+3010540. Overall, this object matches the L5 (or L6) optical standard well (Figure 15). In the near- 


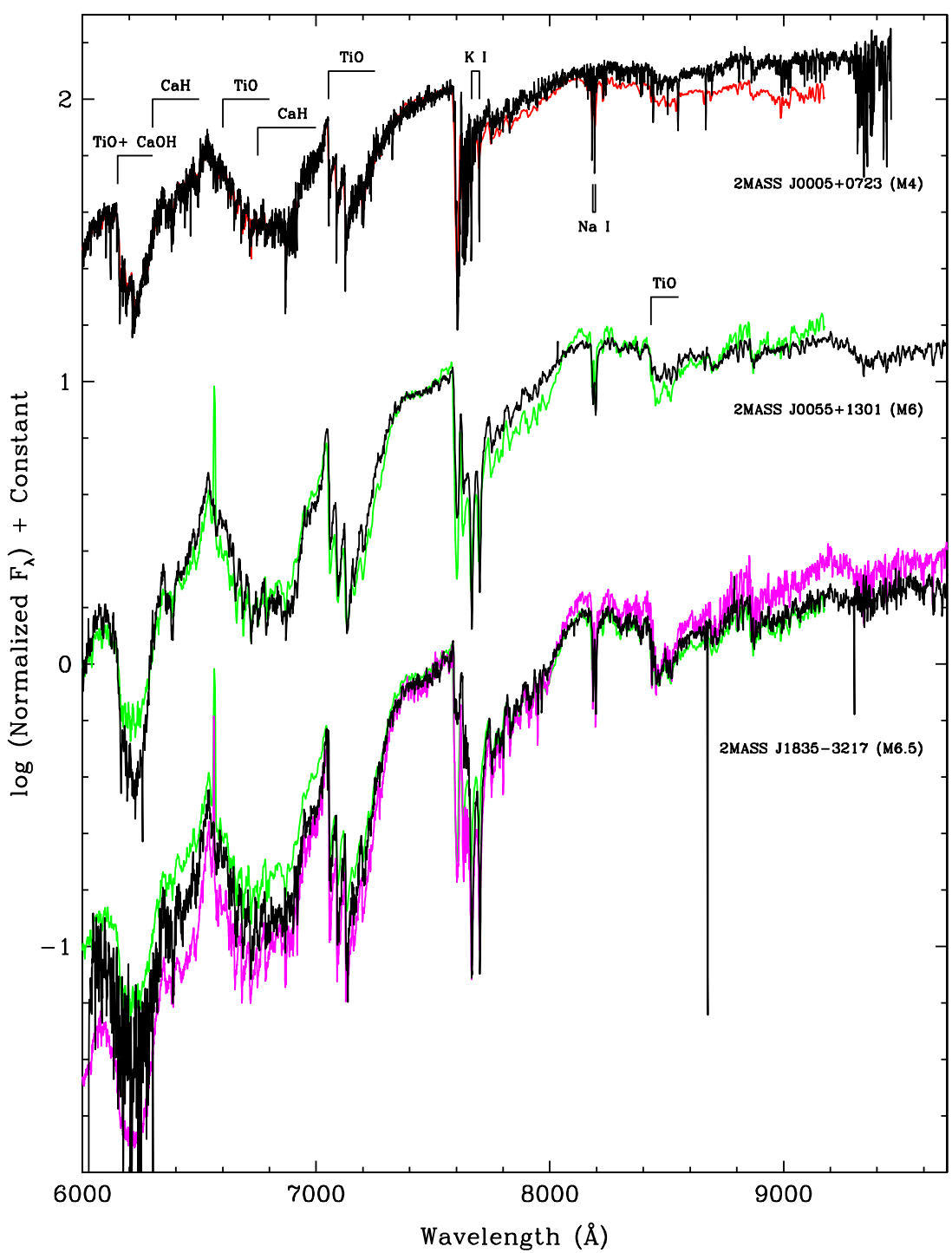

Figure 18. Optical spectra for three objects (black lines) showing intermediate dwarf/subdwarf characteristics in the near-infrared. Overplotted for comparison are optical standards: the M4 dwarf Gl 402 (red), the M6 dwarf Wolf 359 (green), and the M7 dwarf vB 8 (magenta). Spectra are normalized to one at 7500 Å. Integer offsets have been added when needed to separate the spectra vertically.

(A color version of this figure is available in the online journal.)

infrared (Figure 16) its $J$-band spectrum is a good match to the L5 near-infrared standard, but there is excess flux at $H$ - and $K$ bands compared to the standard. Curiously, the 2MASS color of $J-K_{s}=1.831 \pm 0.118 \mathrm{mag}$ places it near the peak color of optically defined L5 or L6 dwarfs in Figure 14 of Kirkpatrick et al. (2008), but the error in this color measurement is large.

These three red L dwarfs along with the other two discoveries published by Looper et al. (2008) have substantial proper motions: $0.44 \pm 0.06,0.24 \pm 0.11,1.39 \pm 0.26,0.37 \pm 0.17$, and $0.28 \pm 0.07 \operatorname{arcsec}^{\mathrm{yr}^{-1}}$ for 2MASS J1331+3407, 2MASS $\mathrm{J} 1821+1414$, 2MASS J2148+4003, 2MASS J2317-4838, and 2MASS J2351+3010, respectively. For estimated distances of roughly $13.8,9.8,9.9,24.4$, and $23.1 \mathrm{pc}$, these correspond to transverse velocities of roughly $28.9,11.2,65.5,43.0$, and $30.8 \mathrm{~km} \mathrm{~s}^{-1}$, respectively. The two most extreme red L dwarfs, 2MASS J2148+4003 and 2MASS J2317-4838, have transverse velocities well above the median transverse velocity of $24.5 \mathrm{~km} \mathrm{~s}^{-1}$ for field L dwarfs (Vrba et al. 2004), but still well below the median transverse velocity of $250 \mathrm{~km} \mathrm{~s}^{-1}$ for
L subdwarfs (a sample of three from Schilbach et al. 2009). Table 13 lists our measured radial velocities and estimates of total space motion for these five objects. Using the median space velocity of the three 2MASS J2148+4003 measures, we find that the average space velocity of this sample is $165 \mathrm{~km} \mathrm{~s}^{-1}$. It therefore appears that these red $\mathrm{L}$ dwarfs are drawn from a considerably older population than the young, low-gravity $\mathrm{M}$ and $\mathrm{L}$ dwarfs discussed in the previous section. Analysis of the spectral energy distribution of 2MASS J2148+4003 by Looper et al. (2008) points to either high metallicity or low gravity as a cause for the spectral peculiarities, but neither of these would be a likely explanation for an older object. It should be noted that our three radial velocity measurements for 2MASS $\mathrm{J} 2148+4003$ are discrepant, ranging over almost $200 \mathrm{~km} \mathrm{~s}^{-1}$, which is a significant variation despite the large uncertainties. Follow-up observations at higher resolution, which may shed additional light on the red L dwarf phenomenon, are desired and easily accomplished since this object is relatively bright (2MASS $J=14.147$ and $\left.K_{s}=11.765\right)$. 


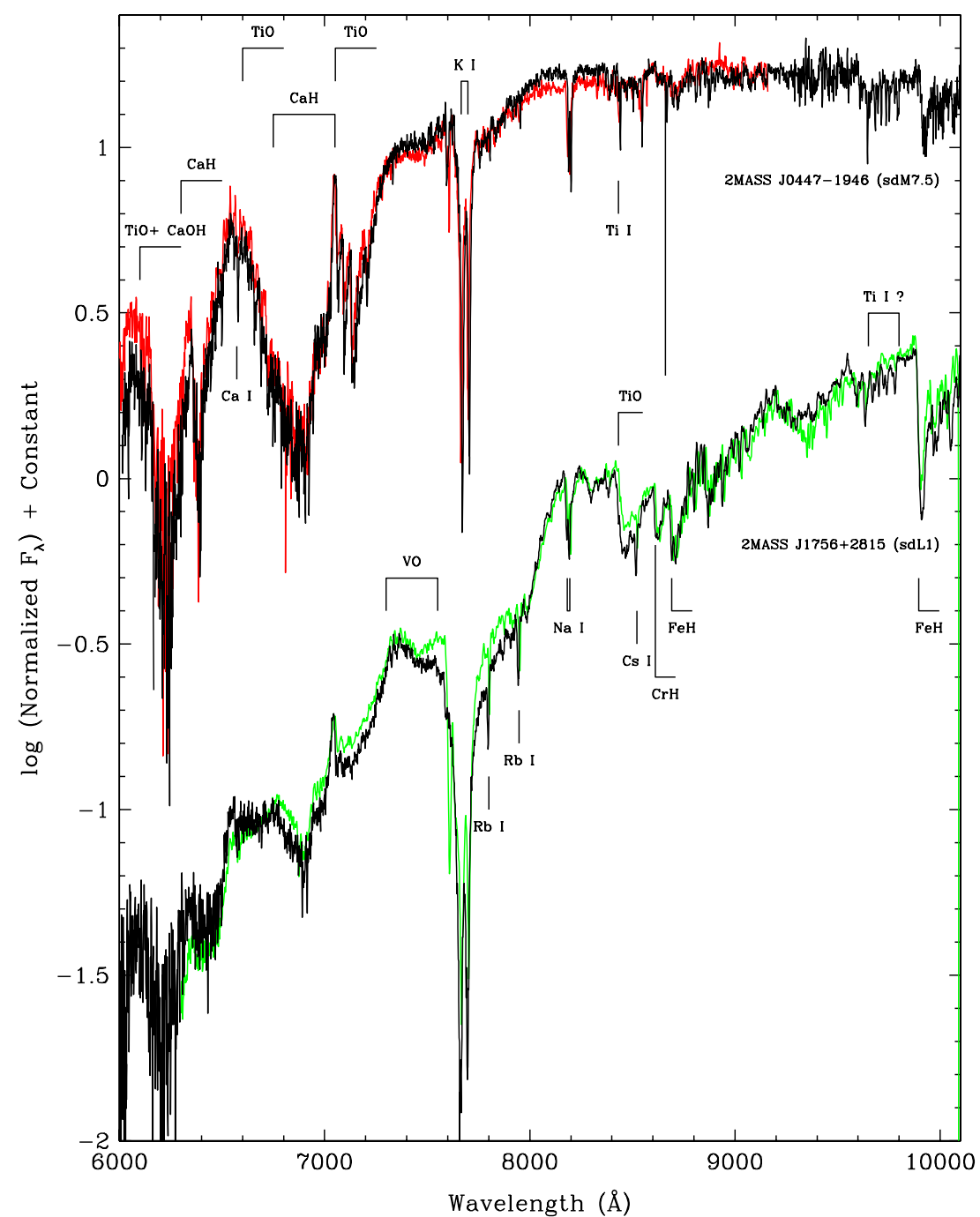

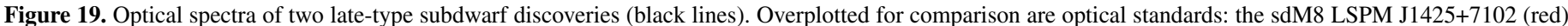
and the L1 dwarf 2MASS J2130-0845 (green). Spectra are normalized to one at $8250 \AA$ and integer offsets added when needed to separate the spectra vertically. (A color version of this figure is available in the online journal.)

\section{3. $M$ and L Subdwarfs}

The Galaxy's oldest constituents are rare in the Solar Neighborhood and sometimes difficult to discern with limited photometric information. Proper motion surveys are particularly well attuned to identifying such objects because of their high kinematics, which result from a long lifetime of gravitational encounters with objects of greater mass (giant molecular clouds, star clusters, etc.). Of particular interest are the low-mass stellar and substellar members of this population, as they contain a fossilized history of star formation near the brown dwarf limit at the Milky Way's earliest epochs. As a group they also contain empirical information about the brown dwarf cooling rate.

Old objects also tend to have low metal content since they are composed of matter devoid of substantial metal enrichment by earlier generations of stars. For the purposes of this paper, we use the term "subdwarfs" to describe those objects whose spectral morphology clearly indicates a metal-poor makeup. The classification of subdwarfs have seen great advancement over the past decade, including the discovery of subdwarfs at and below the hydrogen-burning limit (Gizis 1997; Lépine et al. 2007; Burgasser et al. 2009). Our proper motion survey has identified several new, low-temperature subdwarfs, ranging in type from late-K through late-L. Optical spectra with types of M9.5 or earlier have been classified on the system of Lépine et al. (2007). Because this new system has not yet been extended into the near-infrared and because so few of the extant subdwarfs have been classified on it so far, our near-infrared data are typed using an extension to those wavelengths of the older Gizis (1997) system.

To check for differences between the new Lépine et al. (2007) system and the Gizis (1997) system, we have taken a suite of late-M dwarf and subdwarf optical spectra (Table 3), along with optical spectra of the Lépine et al. (2007) standards (kindly provided by Sebastien Lépine), and typed them according to the Lépine et al. (2007) prescription. Of the thirty new standards, we recover the Lépine et al. (2007) prefix class (sd, esd, or usd) for all thirty. We recover the spectral subclass (K7, M2.5, $\mathrm{M} 7$, etc.) for twenty-four out of thirty; for the remaining six, our computed type is only a half-subclass different from the Lépine et al. (2007) subclass (e.g., we obtain a classification of usdM5.5 as opposed to the published type of usdM5). This sample of spectra, however, contains no normal dwarfs.

When the Lépine et al. (2007) prescription is run on the spectroscopic sample of Table 3, we find the following. As 


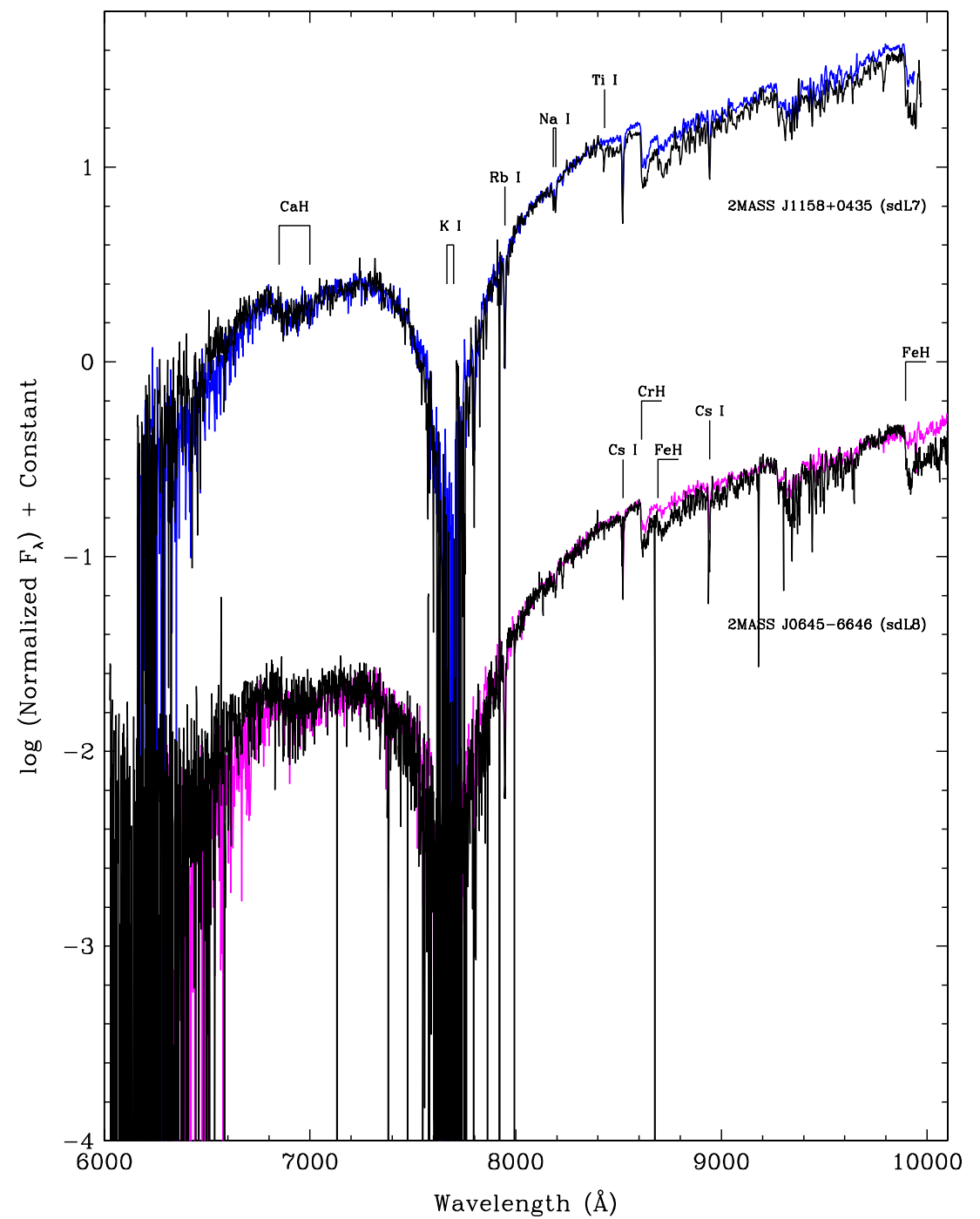

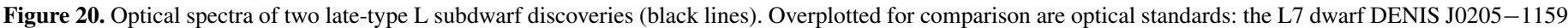

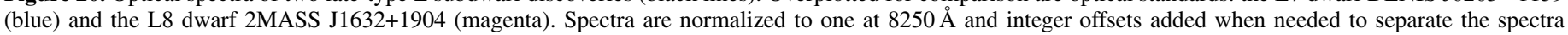
vertically.

(A color version of this figure is available in the online journal.)

expected, all of the normal $\mathrm{M}$ dwarfs were identified as such, although the Lépine et al. (2007) system does not specify a way to obtain the subclass of dM stars. ${ }^{25}$ Of seven objects previously classified as extreme subdwarfs ("esd"), six retain their "esd" classifications. One is reclassified as an ultrasubdwarf ("usd"), a distinction which is new to the Lépine et al. (2007) system. Subtypes sometimes vary by a full subclass or more between the old and new esdM (or usdM) classifications, but this is not surprising given that earlier published types are themselves not always homogeneously classified. More surprising, however, is the fact that of the ten previously typed sdM's (not including LSR J1610-0040, which is a true oddball; Cushing \& Vacca 2006), only four warrant a classification of sdM on the new system.

The six objects that fail to earn a subdwarf classification on the Lépine et al. (2007) system are shown in Figure 17. In two cases-LHS 3189 and LP 97-817-a comparison of their spectra to those of normal dwarfs confirms that types of

\footnotetext{
25 This may be because the weakening of TiO due to dust formation in the later types makes the classification with respect to $\mathrm{TiO}$ and $\mathrm{CaH}$ bands ambiguous beyond M6 (Reid et al. 1995).
}

dM, not sdM, are justified. In the other four cases-LHS 377, LHS 1035, LHS 1135, and LHS 2067-there appears to be sufficient evidence to classify these objects as something other than normal M dwarfs, since they appear to have evidence of low metallicity as demonstrated by their stronger bandstrengths of $\mathrm{CaH}$ relative to $\mathrm{TiO}$. For these four objects, we suggest types of "d/sdM" to denote a morphology intermediate between normal dwarfs and sdMs. These are used as the adopted types in Table 3.

Subdwarf discoveries from our proper motion survey are listed in Table 7 and discussed below. Optical spectra are shown in Figures 18-20. Near-infrared spectra are shown in Figures 21-27.

\subsubsection{Borderline Subdwarfs}

Three objects have near-infrared spectra which support a type intermediate between normal M7 and sdM7 (i.e., d/sdM7) ${ }^{26}$. We also have optical spectra for two of these objects, and in both cases a normal dwarf spectral type, not a subdwarf type, appears warranted.

\footnotetext{
26 Note that the near-infrared sdM7 being plotted is LHS 377, an object
} classified in the optical (see discussion above) as d/sdM5. 


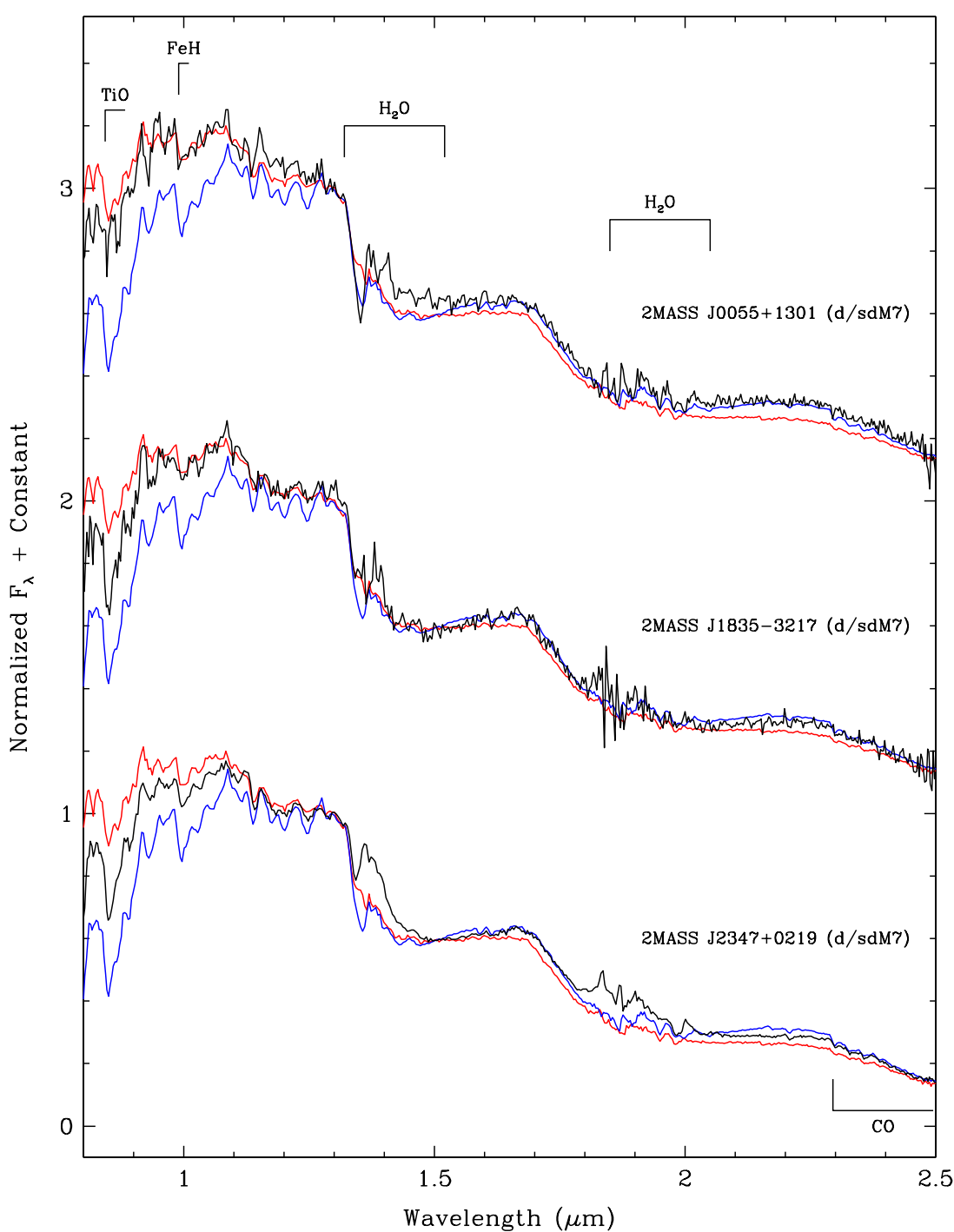

Figure 21. Near-infrared spectra of three discoveries having near-IR classifications intermediate between M7 and sdM7 (black lines). Overplotted for comparison are the optically classified sdM7 LHS 377 (red) and the near-infrared M7 standard vB8 (blue). Spectra are normalized to one at $1.28 \mu \mathrm{m}$ and integer offsets added when needed to separate the spectra vertically.

(A color version of this figure is available in the online journal.)

2MASS J00554279+1301043. Whereas the optical spectrum of this object (Figure 18) supports a type of M6, the near-infrared spectrum supports a type intermediate between M7 and sdM7 (Figure 21).

2MASS J18355309-3217129. Whereas the optical spectrum of this object (Figure 18) supports a type of M6.5, the nearinfrared spectrum supports a type intermediate between M7 and sdM7 (Figure 21).

2MASS J23470713+0219127. The near-infrared spectrum supports a type intermediate between M7 and sdM7 (Figure 21). We have no optical spectrum for this object.

\subsubsection{Late-M Subdwarfs}

2MASS J00054517+0723423. In the optical the spectrum of this object matches a normal M4 dwarf (Figure 18). The nearinfrared spectrum of this object (Figure 22), however, best fits an sdM6. Note that the near-infrared spectrum of the M4 standard provides a poor match, as it shows pronounced differences below $1 \mu \mathrm{m}$ and its infrared water bands are too shallow.

2MASS J04470652-1946392. This is our latest M-type subdwarf discovery. Figure 19 shows the optical spectrum compared to that of the sdM8 standard from Lépine et al. (2007). The overall bandstrengths and continua are very similar. In addition to the strong hydride bands of $\mathrm{CaH}$ near 6350 and $6950 \AA$ and $\mathrm{FeH}$ band at $9896 \AA$, this spectrum also shows the late subdwarf hallmarks of strong metal lines-Ca I at $6517 \AA$, Ti I (7209/7213, 8433, and 9600-9700 $\AA$ ), and Ca II (8498, 8542, and $8662 \AA)$. Using the Lépine et al. (2007) $\zeta_{\mathrm{TiO} / \mathrm{CaH}}$ metallicity index along with the relation between the $\mathrm{CaH} 2$ and $\mathrm{CaH} 3$ indices and spectral type, we classify this object in the optical as an sdM7.5. In the near-infrared (Figure 22) this object best matches the spectrum of LSR J2036+5100, which has also been classified as an sdM7.5. Using the trigonometric parallax of LSR J2036+5100 from Schilbach et al. (2009) and the 2MASS $J$-band magnitudes of LSR J2036+5100 and 2MASS J0447-1946, we estimate a distance to 2MASS J0447-1946 of $102 \mathrm{pc}$.

2MASS J10462067+2354307. The near-infrared spectrum of this object (Figure 22) best fits an sdM6. We have no optical spectrum.

$2 M A S S$ J16130315+6502051. The near-infrared spectrum of this object (Figure 22) best fits an sdM6. Again, we have no optical spectrum. 


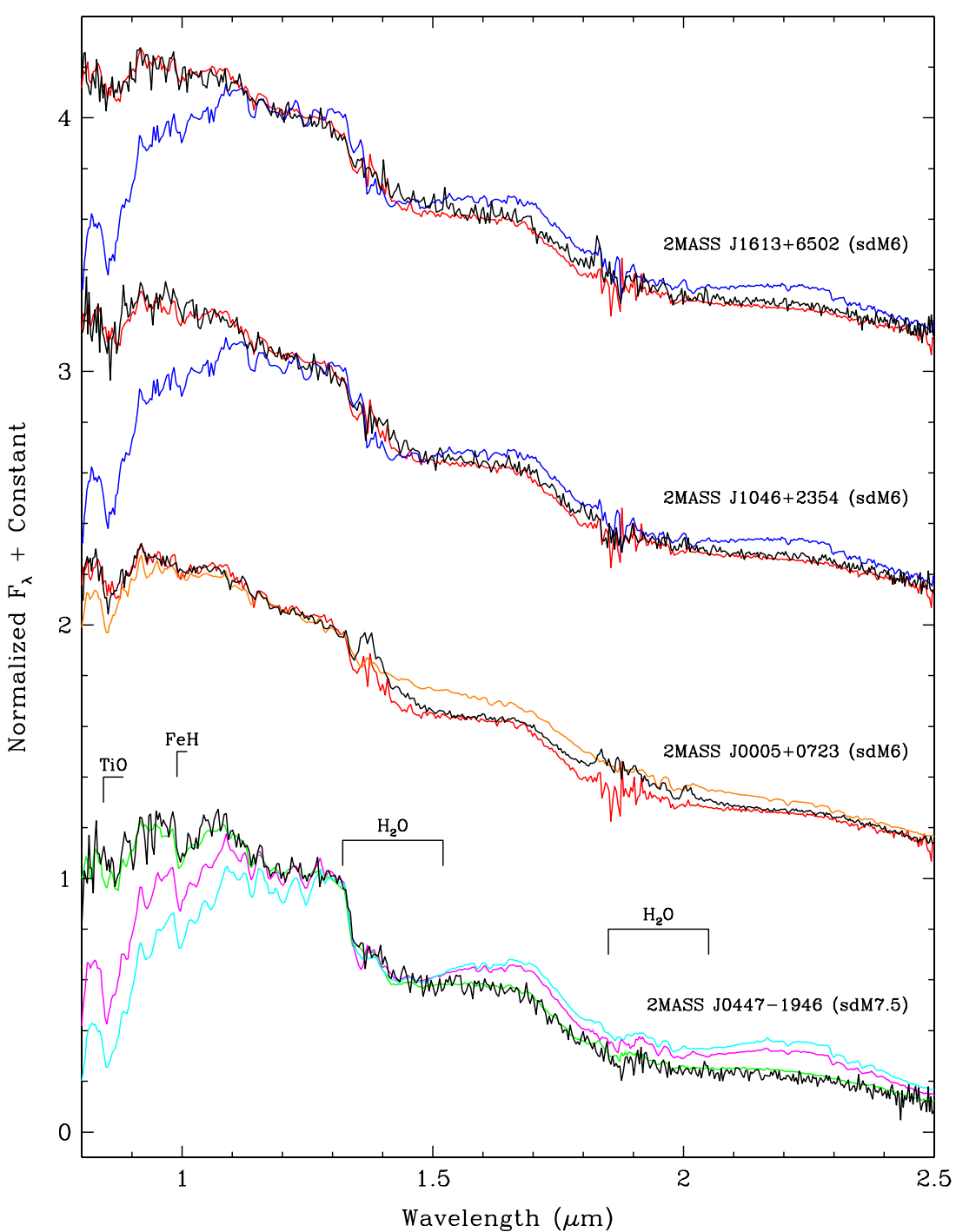

Figure 22. Near-infrared spectra of four late-M subdwarf discoveries (black lines). Overplotted for comparison are near-infrared spectra of the optically classified sdM6 LHS 1074 (red) and the optically classified sdM7.5 LSR J2036+5100 (green). Also plotted for comparison are near-infrared M dwarf standards: the M4 Gl 213 (orange), the M6 LHS 3933 (blue), the M7 vB 8 (magenta), and the M8 vB 10 (cyan). Spectra are normalized to one at $1.28 \mu \mathrm{m}$ and integer offsets added when needed to separate the spectra vertically.

(A color version of this figure is available in the online journal.)

\subsubsection{Subdwarfs}

Our discovery of three new L subdwarfs substantially increases the number of known members of this class (Table 8). Because there are few known examples, there are no classification anchors on which to pin spectral types, so the closest match to the normal L dwarf sequence is used instead.

2MASS J06453153-6646120. This is the latest subdwarf discovery from our survey. The optical spectrum, shown in Figure 20, most closely matches an L8 dwarf but there are peculiarities. Because the $\mathrm{FeH}$ and $\mathrm{CrH}$ bands are noticeably weaker in 2MASS J0645-6646 than in the L8 standard, we label this as an sdL8. In the near-infrared (Figure 23), 2MASS J0645-6646 shows a markedly blue continuum compared to a normal L8. The curious absorption trough just shortward of $1.6 \mu \mathrm{m}$ (Figure 27) appears to be enhanced $\mathrm{FeH}$, echoing the strong hydride bands seen in the optical. The 2MASS color of $J-K_{s}=1.259 \pm 0.050 \mathrm{mag}$ is bluer than all of the optically defined L8 dwarfs in Figure 14 of Kirkpatrick et al. (2008) and $\sim 0.5$ mag bluer than the median L8 color. At $\mu=$
$1.57 \pm 0^{\prime \prime} 08 \mathrm{yr}^{-1}, 2 \mathrm{MASS}$ J0645-6646 is the highest proper motion object found in the 1X-6X survey. Using its 2MASS $J$-band magnitude and assuming its absolute $J$-band magnitude is comparable to that of a normal L8 (Looper et al. 2008), we estimate a distance to 2MASS J0645-6646 of $\sim 16 \mathrm{pc}$.

2MASS J11582077+0435014. The optical spectrum of this object, shown in Figure 20, most closely matches a normal L7 but bands of $\mathrm{TiO}, \mathrm{FeH}$, and $\mathrm{CrH}$ are much stronger in 2MASS $\mathrm{J} 1158+0435$. For this reason, we classify the object as an sdL7. In addition to the strong alkali lines seen in late-L dwarfs, Ti I at $8433 \AA$ is also seen and this is another oddity sometimes seen in late-type subdwarfs. In the near-infrared (Figure 23) the best overall match to the suite of standard L dwarfs from Figure 10 is the L7 but there are marked differences. At $J$ band the $\mathrm{FeH}$ bands are much stronger than in the normal L7 and the overall continuum at $H$ and $K$ bands is markedly suppressed. The 2MASS $J-K_{s}$ color of this object, $1.172 \pm$ $0.084 \mathrm{mag}$, is bluer than all of the optically defined L7 dwarfs in Figure 14 of Kirkpatrick et al. (2008) and $\sim 0.6$ mag bluer than the median L7 color. A detail of the NIRSPEC N3 spectrum of 


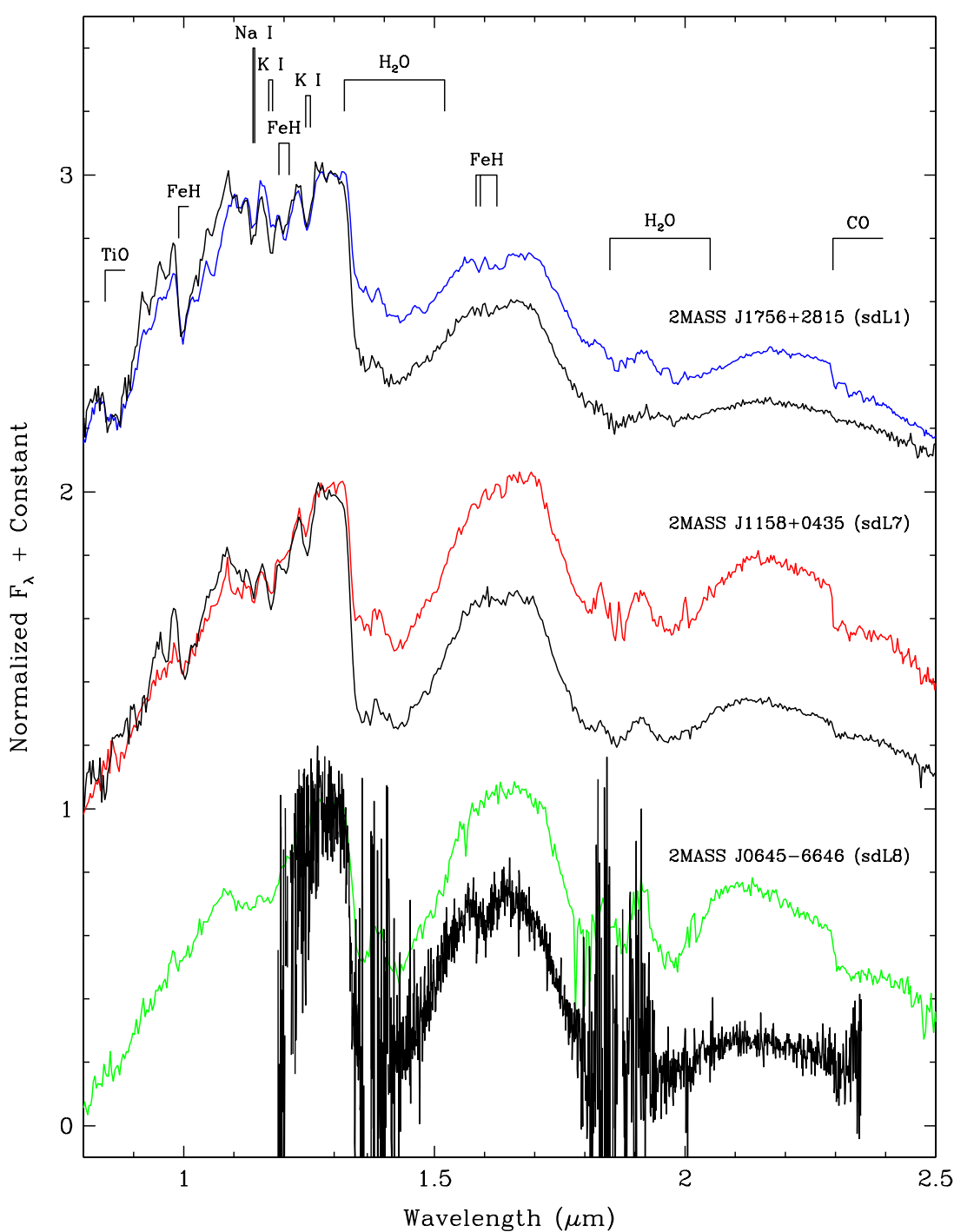

Figure 23. Near-infrared spectra of three subdwarf L discoveries (black lines). Overplotted for comparison are near-infrared standards: the L1 dwarf $2 \mathrm{MASS}$ J2130-0845 (blue), the L7 dwarf 2MASS J0103+1935 (red), and the L8 dwarf 2MASS J1632+1904 (green). Spectra are normalized to one at $1.28 \mu \mathrm{m}$ and integer offsets added when needed to separate the spectra vertically.

(A color version of this figure is available in the online journal.)

this object is shown in Figure 26. Both the FeH bands and the $\mathrm{K}$ I doublet lines are stronger than in the L7 standard. In lieu of a trigonometric parallax, we estimate a distance of $\sim 16 \mathrm{pc}$ to 2MASS J1158+0435 using its 2MASS $J$-band magnitude and the absolute $J$-band magnitude of a normal L 7 from Looper et al. (2008).

2MASS J17561080+2815238, In the optical, this object most resembles an L1 dwarf but shows several discrepancies. Figure 19 illustrates that 2MASS J1756+2815 has stronger TiO, $\mathrm{FeH}$, and $\mathrm{CrH}$ bands than the standard L1, leading us to assign a classification of sdL1. Note also that 2MASS J1756+2815 has stronger $\mathrm{K}_{\mathrm{I}}$ line cores and, particularly, line wings than the L1 standard. Lines of $\mathrm{Rb}$ I and Cs I are also stronger. In the near-infrared (SpeX data in Figure 23; NIRSPEC data in Figure 24) the most striking feature is the suppression of the $H$ - and $K$ bands relative to a normal $\mathrm{L} 1 \mathrm{dwarf}$. Also noticeable are its stronger lines of $2.20 \mu \mathrm{m} \mathrm{NaI}$ and stronger bands of $2.30 \mu \mathrm{m} \mathrm{CO}$ at $K$ band. Its 2MASS $J-K_{s}$ color of $0.899 \pm$ 0.053 mag is bluer than all of the optically defined L1 dwarfs in Figure 14 of Kirkpatrick et al. (2008) and $\sim 0.4$ mag bluer than the median L1 color. A detail of the NIRSPEC N3 spectrum of this object is shown in Figure 25. Note that the K I doublet lines are stronger and broader than in the L1 standard although, curiously, the strengths of the $\mathrm{FeH}$ bands here are not markedly different. Because of this, we are not able to classify this object in the near-infrared as an L subdwarf, but denote it as a blue L1. (Blue L dwarfs are discussed further below.) This is the only object so far identified that has this strongly dual nature, so it is worthy of concentrated follow-up work. Using the 2MASS $J$-band magnitude and the Looper et al. (2008) relation noted above, we estimate a distance of $\sim 35 \mathrm{pc}$.

\subsubsection{Discussion of L Subdwarfs}

Table 13 lists the radial velocities, tangential velocities, and total space motions measured for the three new $\mathrm{L}$ subdwarfs and one late-M subdwarf. We find an average tangential velocity of $171.5 \mathrm{~km} \mathrm{~s}^{-1}$, or an average space motion of $223 \mathrm{~km} \mathrm{~s}^{-1}$, for this sample of four. Although high, this velocity is not as high as the average tangential velocity of $263.5 \mathrm{~km} \mathrm{~s}^{-1}$ found for late-M and L subdwarfs in Schilbach et al. (2009). Given that the L subdwarfs comprising the bulk of the Schilbach et al. (2009) sample are much bluer than those found here-mainly because 


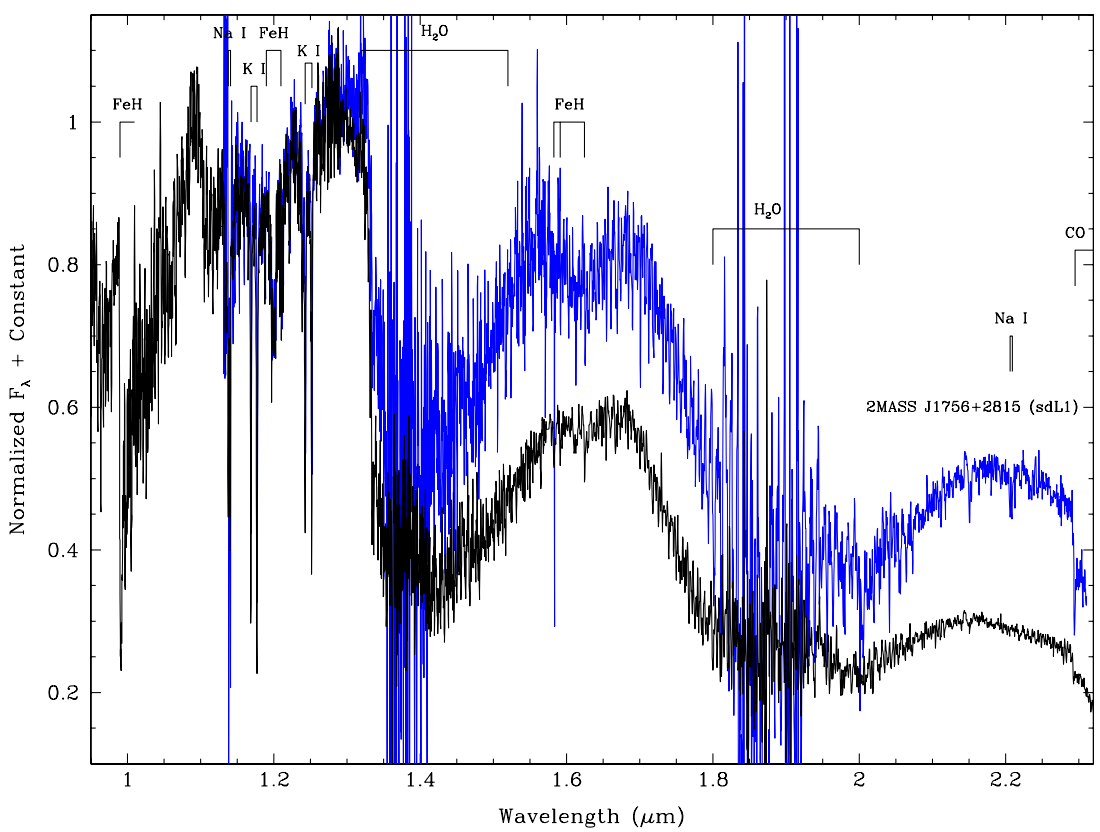

Figure 24. NIRSPEC data for the sdL1 2MASS J1756+2815 (black line) compared to the normal L1 dwarf 2MASS J1658+7027 (blue). Spectra are normalized to one at $1.28 \mu \mathrm{m}$.

(A color version of this figure is available in the online journal.)

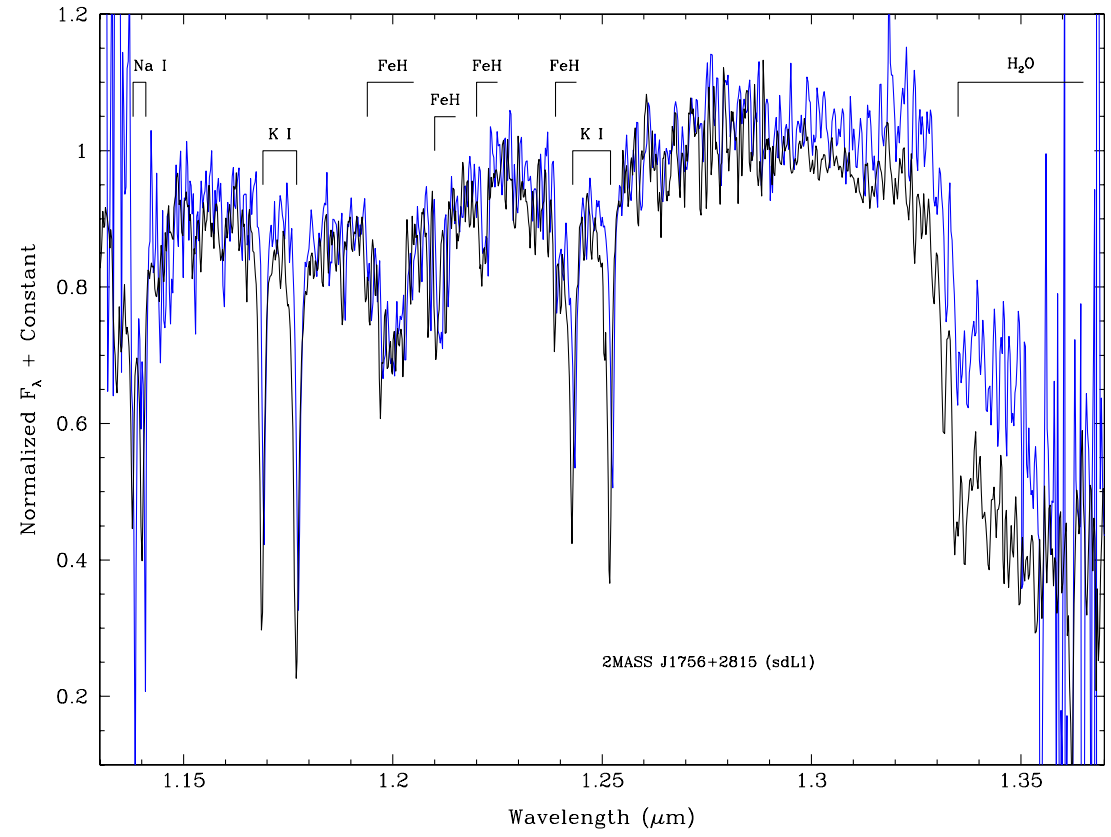

Figure 25. Blow-up of Figure 24 showing the $J$-band portion from 1.13 to $1.37 \mu \mathrm{m}$. Spectra are normalized to one at $1.28 \mu \mathrm{m}$.

(A color version of this figure is available in the online journal.)

the earliest L subdwarf discoveries were made as by-products of T dwarf searches focused on very blue objects in $J-K_{s}$ color-they may also represent a substantially more metal poor and older population than those found here. Nonetheless, our new L subdwarf discoveries are kinematically distinct from the field L dwarf population, whose median transverse velocity is $24.5 \mathrm{~km} \mathrm{~s}^{-1}$ (Vrba et al. 2004).

There are several reasons to believe that these three L subdwarfs, with the possible exception of the enigmatic 2MASS $\mathrm{J} 1756+2815$, are objects low in metallicity. (1) Stronger hydride bands are the by-product of an atmosphere where metal-metal molecules such as $\mathrm{TiO}$ or $\mathrm{VO}$ are less abundant. With less absorption by the oxides, the hydride bandstrengths are more prominent. (2) In a cool, metal-deficient atmosphere, the relative absorption by collision-induced $\mathrm{H}_{2}$ is stronger than in an atmosphere with a rich array of lines and bands from metalbearing species. Because this broad $\mathrm{H}_{2}$ absorption is strongest at $H$ - and $K$ bands (Borysow et al. 1997), this leads to a bluer near-infrared continuum, and hence a bluer $J-K_{s}$ color, for L subdwarfs when compared to L dwarfs of higher metallicity (Burgasser et al. 2003). (3) Somewhat counterintuitively, the presence of metal lines (such as Ti I and Ca I) and the increased strengths of the alkali lines (NaI, KI, Cs I, and RbI) in lowtemperature atmospheres may indicate retarded condensate 


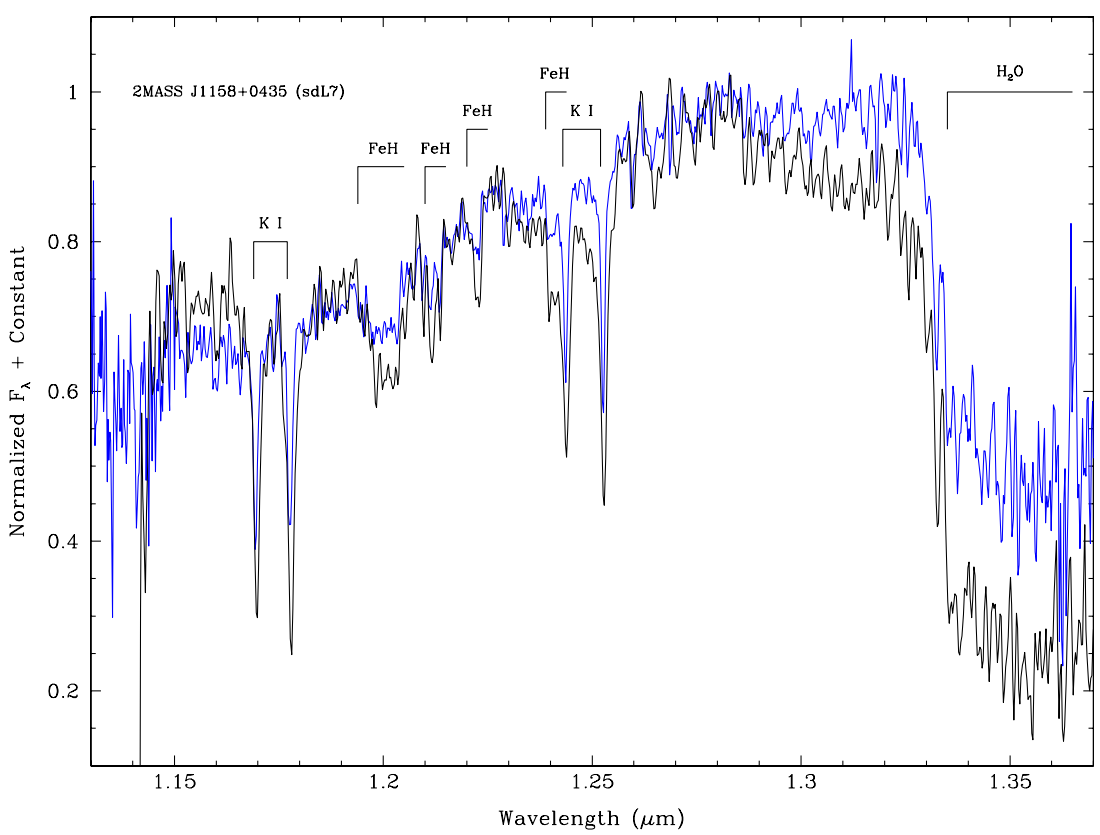

Figure 26. NIRSPEC data for the sdL7 2MASS J1158+0435 (black line) compared to the standard L7 dwarf 2MASS J0103+1935 (blue). Spectra are normalized to one at $1.28 \mu \mathrm{m}$.

(A color version of this figure is available in the online journal.)

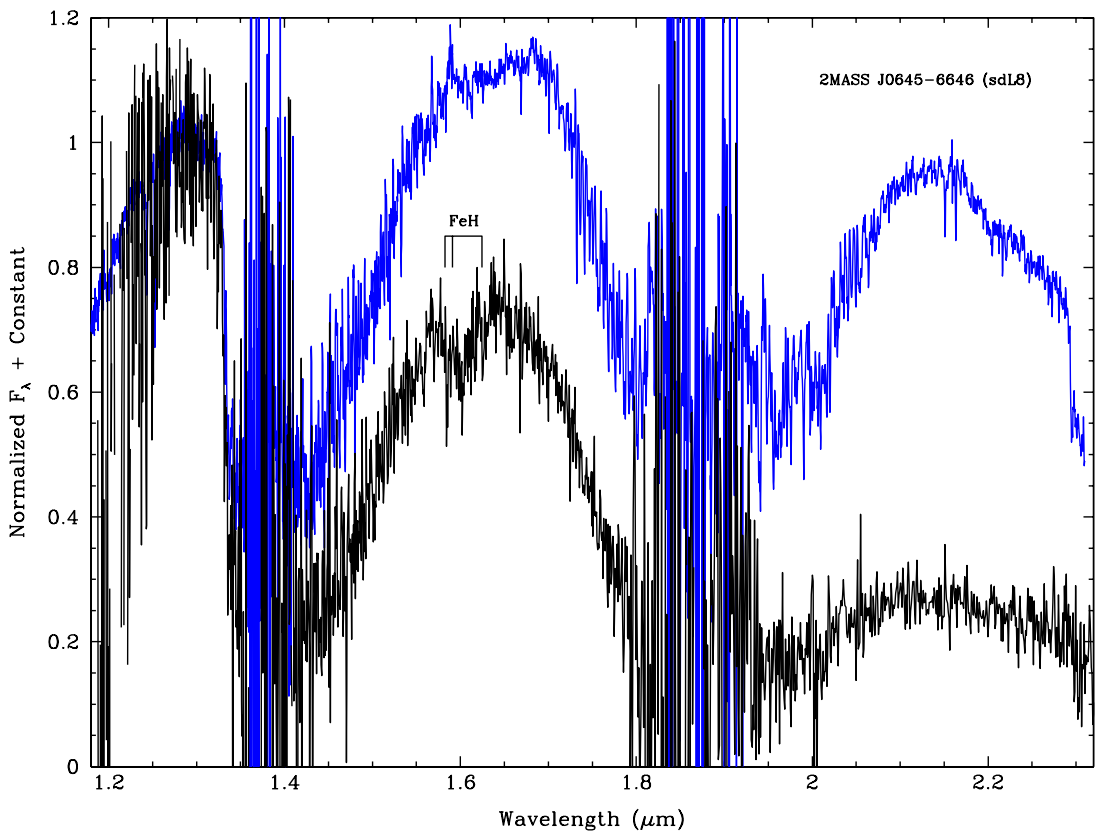

Figure 27. Blow-up of the OSIRIS spectrum of the sdL8 discovery 2MASS J0645-6646 (black line) compared to the NIRSPEC spectrum of the near-infrared L8 standard 2MASS J1632+1904 (blue). Both spectra have been normalized to one at $1.28 \mu \mathrm{m}$.

(A color version of this figure is available in the online journal.)

formation in a metal-poor environment. The rarity of metal atoms in the atmosphere means that condensates are more difficult to form, and thus these metals remain in the atmosphere to lower temperatures.

Using one of these new discoveries (2MASS J1158+0435) along with a new discovery from the SDSS team (SDSS J1416+1348; Schmidt et al. 2010), we are able to build an empirical sequence in metallicity for spectral type L7. Figures 28 and 29 show the optical and near-infrared spectra of these two objects compared to the standard L7, DENIS J0205-1159 (presumably of solar metallicity), and the first late-L subdwarf dis- covered, 2MASS J05325346+8246465. 2MASS J1158+0435 and SDSS J1416+1348 are very similar in spectral appearance, although the SDSS object shows slightly stronger hydride bands $(\mathrm{CaH}, \mathrm{CrH}$, and $\mathrm{FeH})$, slightly stronger $\mathrm{TiO}$, and a bluer near-infrared continuum (by $0.14 \mathrm{mag}$ in $J-K_{s}$ ). The spectrum of 2MASS J0532+8246 is even more extreme than these, particularly in the strength of its $\mathrm{CaH}$ band and in its overall near-infrared color, which is 0.77 mag bluer than in SDSS J1416+1348. (The slightly weaker $\mathrm{TiO}, \mathrm{CrH}$, and $\mathrm{FeH}$ bands in 2MASS J0532+8246 may indicate that this object is a bit later in type than the other two subdwarf comparisons.) 

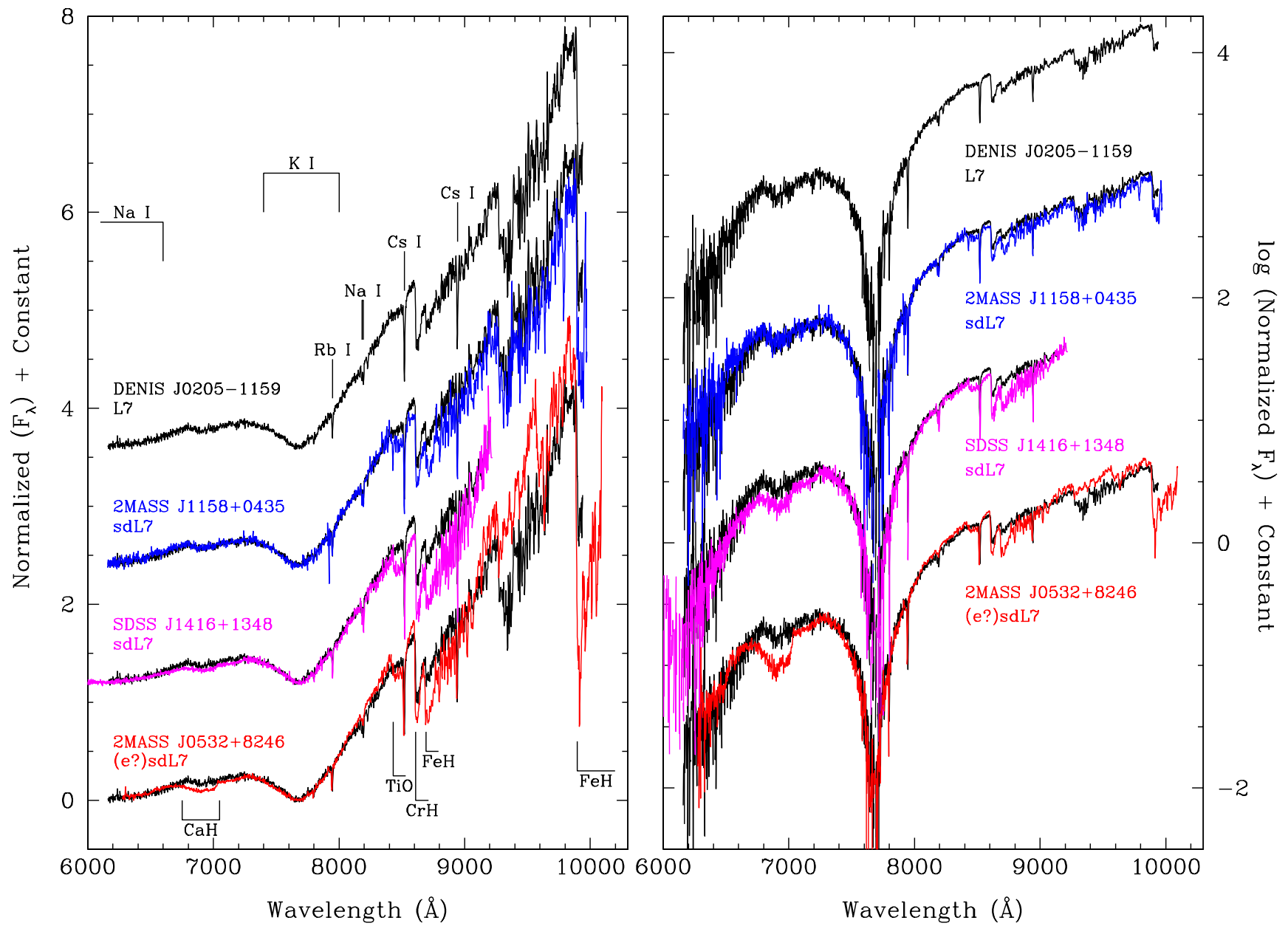

Figure 28. L7 metallicity sequence at optical wavelengths. Shown are the optical L7 dwarf standard DENIS J0205-1159 (black line) from Kirkpatrick et al. (1999), the new sdL7 2MASS J1158+0435 (blue) from this paper, the bright sdL7 SDSS J1416+1348 (magenta) from Schmidt et al. (2010), and the more extreme L7 subdwarf 2MASS J0532+8246 (red) from Burgasser et al. (2003). Spectra have been normalized to one at $8250 \AA$ and offsets in multiple units of 1.2 have been added to space the spectra vertically. The left panel shows the flux in linear units and the right panel shows the flux in logarithmic units.

(A color version of this figure is available in the online journal.)

Because of its more extreme nature, we propose that 2MASS J0532+8246 should actually be labeled as an esdL7 to indicate an even more unusual spectral morphology. Further investigation will be needed to determine if such morphological traits easily translate into a lower value of $[\mathrm{Fe} / \mathrm{H}]$. In one example, the sdL5 2MASS J06164006-6407194 has a spectral morphology that is not nearly as abnormal as that of 2MASS J0532+8246 yet kinematic investigations place the former in the outer halo (Cushing et al. 2009) and the latter in the inner halo (Burgasser et al. 2008). Single objects may not be representative of their population, however, so larger collections of L subdwarfs will be needed to further gauge the variation in spectral appearance of these two, very old samples.

\subsection{Unusually Blue L Dwarfs}

Although L subdwarfs have particularly blue $J-K_{s}$ colors due to increased relative collision-induced opacity by $\mathrm{H}_{2}$ at $H$ and $K_{s}$ bands, there is a class of $\mathrm{L}$ dwarfs that shows unusually blue near-infrared colors not obviously caused by low metallicity. These so-called blue $\mathrm{L}$ dwarfs have unusually blue $J-K_{s}$ colors for their optical spectral types and were first pointed out in follow-up of 2MASS sources by Gizis et al. (2000) and Cruz et al. (2003). Subsequent follow-up of SDSS sources revealed objects with peculiar near-infrared spectral morphologies. Specifically, spectral indices measuring the depth of $\mathrm{H}_{2} \mathrm{O}$ bands versus those measuring $\mathrm{FeH}$ or $\mathrm{K}$ I give discordant spectral types, with the $\mathrm{H}_{2} \mathrm{O}$-based index always suggesting a much later L subclass (Knapp et al. 2004; Chiu et al. 2006). Some of these blue $\mathrm{L}$ dwarfs are suspected to be the composite spectra of an L dwarf + Tdwarf binary (e.g., Burgasser 2007). One intriguing blue L dwarf, 2MASS J11263991-5003550, for which binarity seems not to be the cause, has been extensively studied by Folkes et al. (2007) and Burgasser et al. (2008). The latter authors argue that the spectral features are the result of thin and/or large-grained clouds, the cause for which appears not to be easily attributable to either surface gravity or low metallicity. Another example-an object known as 2MASS $\mathrm{J} 17114559+4028578$ (G 203-50B) -is particularly interesting because it shares common proper motion with an M dwarf whose optical spectrum suggests a metallicity very similar to that of the Sun (Radigan et al. 2008).

Faherty et al. (2009) performed a kinematic study of 10 unusually blue $\mathrm{L}$ dwarfs and found a median transverse velocity of $99 \pm 47 \mathrm{~km} \mathrm{~s}^{-1}$, substantially larger than that of the field L dwarf population $\left(24.5 \mathrm{~km} \mathrm{~s}^{-1}\right.$; Vrba et al. 2004) but still smaller than the transverse velocity for M subdwarfs (196 \pm $91 \mathrm{~km} \mathrm{~s}^{-1}$ ). This suggests that many of the blue L dwarfs may be old, though not as old as the halo population. It therefore 


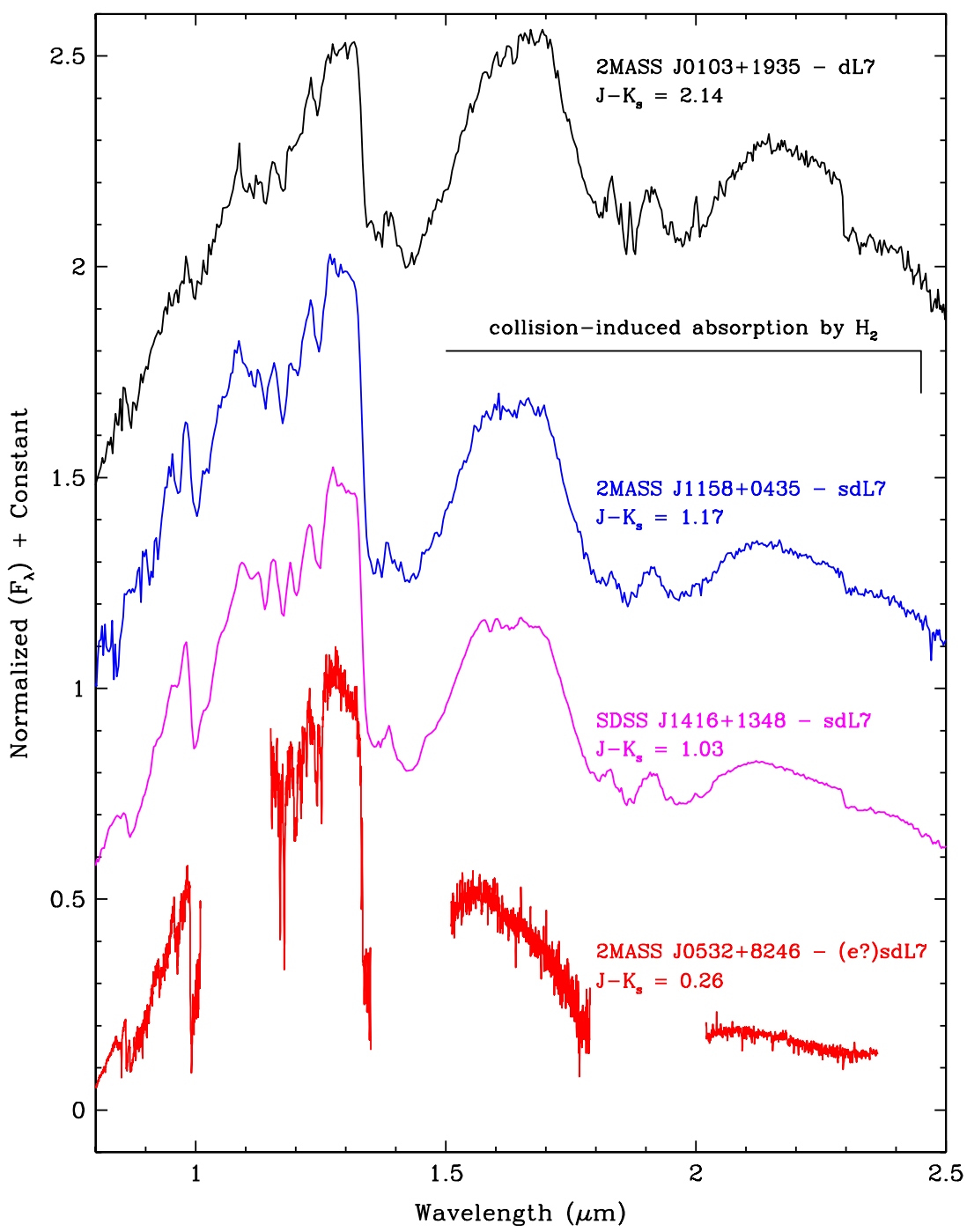

Figure 29. L7 metallicity sequence at near-infrared wavelengths. Color coding is the same as in Figure 28. Spectra have been normalized to $1.28 \mu \mathrm{m}$, and vertical offsets are in multiple units of 0.5 .

(A color version of this figure is available in the online journal.)

remains a possibility that these objects represent a slightly lowermetallicity population than normal field dwarfs but not so low in metal content that they can be confidently labeled as subdwarfs. For very cool atmospheres such as these, a small change in metal content can perhaps have more prominent effects on the formation of condensates (i.e., cloud properties) than on the strength of gas phase molecular transitions.

As a result of our proper motion study, we report another eight blue L dwarfs (Table 9), spectra for which are shown in Figures 30-34, For the purposes of this paper, we define a "blue L dwarf" to be an object whose $1.5-2.5 \mu \mathrm{m}$ continuum is significantly bluer than expected, given the continuum shape of the near-infrared spectroscopic standard that best fits its spectrum at $J$ band, but whose cause is not obviously low metallicity as judged primarily by the strength of hydride features. We discuss each of these eight objects below:

2MASS J00150206+2959323. The optical spectrum of this object (Figure 31 ) is very similar to the L7 optical standard except that the $\mathrm{FeH}$ and $\mathrm{CrH}$ bandstrengths are somewhat stronger in 2MASS J0015+2959. Our near-infrared spectrum (Figure 34) is best fit by either the L7 or the L8 near-infrared standard. The depth of the water band between the $J$ and $H$ bands is well matched by the L8 standard, but the flux peaks at $H$ and $K$ bands in 2MASS J0015+2959 are significantly suppressed relative to this same standard. This continuum suppression is even more extreme at $K$ band if the L7 standard is used as the comparison. The 2MASS $J-K_{s}$ value of 1.676 \pm 0.109 mag is only $\sim 0.15$ mag bluer than the median color of other optically defined L7 dwarfs (Kirkpatrick et al. 2008), although the uncertainty in this measurement is large.

2MASS J03001631+2130205. Our near-infrared spectrum (Figure 33) is an excellent match to the L6 near-infrared standard in the $J$ band. At $H$ and $K$ bands, however, the continuum is markedly bluer than that of the standard, earning 2MASS J0300+2130 a "blue L" classification. Its 2MASS $J-K_{s}$ color of $1.566 \pm 0.098 \mathrm{mag}$ is $\sim 0.35 \mathrm{mag}$ bluer than the median color for other optically defined L6 dwarfs.

2MASS J10461875+4441149. We have only a near-infrared spectrum of this object (Figure 33) but it shows a bluer continuum at wavelengths longward of $1.5 \mu \mathrm{m}$ compared to the L5 near-infrared standard that it best matches shortward of this. Except for this, the overall feature strengths through the spectrum look very similar to the standard itself. 


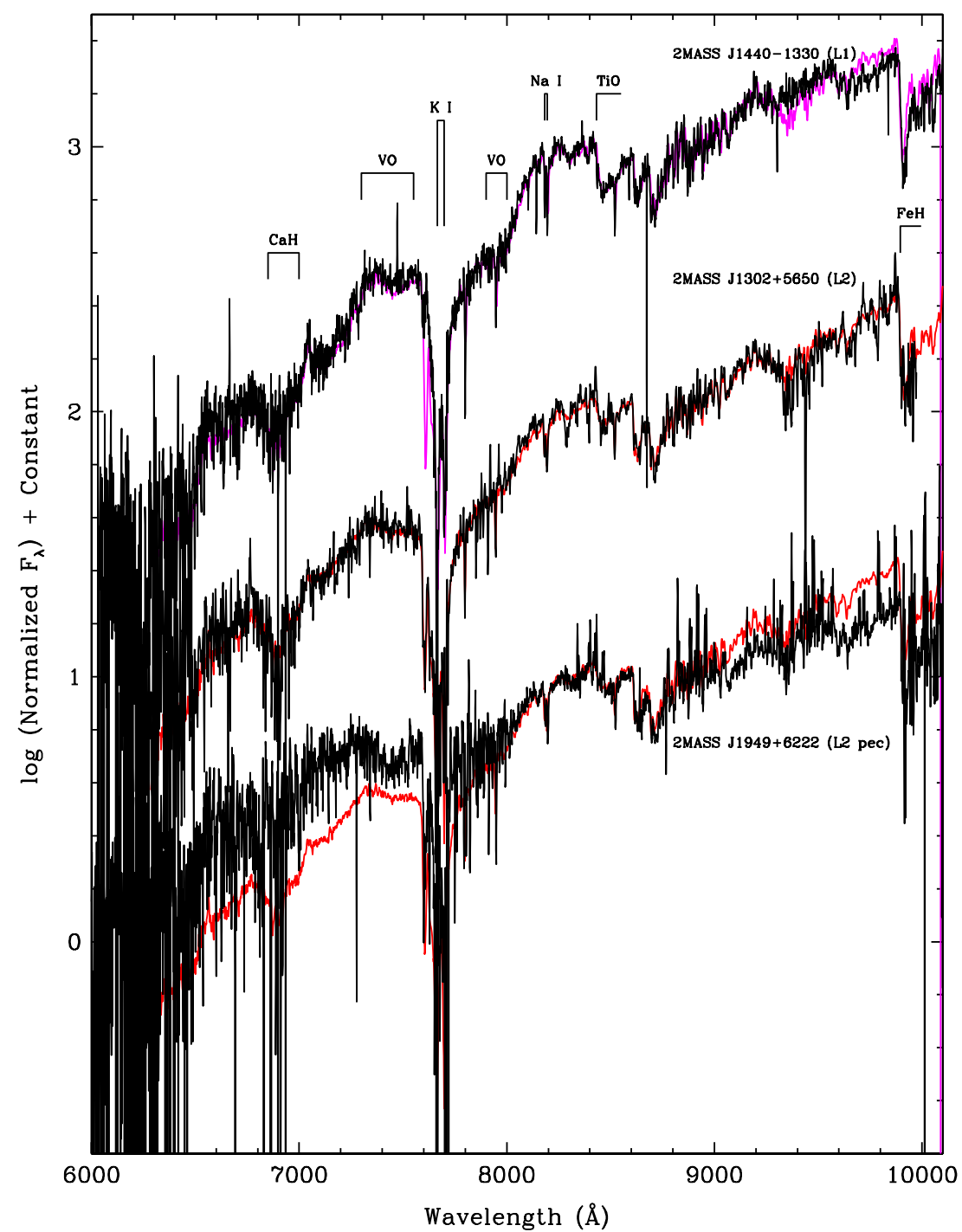

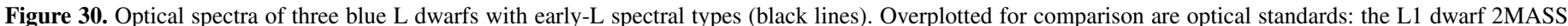

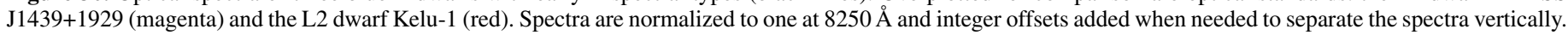
(A color version of this figure is available in the online journal.)

2MASS J11181292-0856106. Figure 31 shows the optical spectrum, which is a very good match to the optical L6 standard. In the near-infrared (Figure 33), the best $J$-band fit is again to the L6 standard, but the overall bandstrengths do not match well. At $H$ - and $K$ bands the flux in 2MASS J1118-0856 is considerably quenched relative to the L6 standard. Its 2MASS $J-K_{s}$ color of $1.560 \pm 0.102 \mathrm{mag}$ is $\sim 0.35 \mathrm{mag}$ bluer than the median color for other optically defined L6 dwarfs.

2MASS J13023811+5650212. The optical spectrum, shown in Figure 30, is very similar to the L2 optical standard. Our nearinfrared spectrum (Figure 32) is noisy but shows a suppressed continuum at $H$ and $K$ bands relative to the near-infrared L3 standard that it best matches at $J$ band. Its 2MASS $J-K_{s}$ color of $1.388 \pm 0.173 \mathrm{mag}$ is only $\sim 0.1 \mathrm{mag}$ bluer than the median color for other optically defined L2 dwarfs, but the uncertainty on this color measure is large.

2MASS J14403186-1303263: The optical spectrum, shown in Figure 30, is very similar to the L1 optical standard. The near-infrared spectrum (Figure 32) is most like the L1 nearinfrared standard at $J$ band but shows a suppressed continuum at longer wavelengths. Most striking is the much stronger $\mathrm{H}_{2} \mathrm{O}$ absorption trough between $J$ and $H$ bands. Its 2MASS $J-K_{s}$ color of $1.128 \pm 0.082$ mag is $\sim 0.2$ mag bluer than the median color for other optically defined L1 dwarfs.

2MASS J19495702+6222440. The optical spectrum of this object (Figure 30) best fits the L2 optical standard over the wavelength region from 8000 to $9000 \AA$ but is otherwise much too flat (blue) for an early-L dwarf. Longward of $9000 \AA$, the spectrum of 2MASS J1949+6222 is suppressed relative to the standard, although the $\mathrm{FeH}$ and $\mathrm{CrH}$ bandstrengths are very similar. Shortward of $8000 \AA$ the spectrum of $2 \mathrm{MASS}$ J1949+6222 shows excess flux relative to the standard, and the $7400 \AA$ A band of VO is much stronger than in the standard L2. Our near-infrared spectrum of 2MASS J1949+6222 (Figure 32) is noisy but nonetheless shows a somewhat suppressed continuum at $H$ and $K$ bands relative to the near-infrared L2 standard that it best matches at $J$ band. Surprisingly, its 2MASS-measured $J-K_{s}$ color of $1.609 \pm 0.167 \mathrm{mag}$ is $\sim 0.1$ mag redder than the median color for other optically defined L2 dwarfs, but the photometric error is substantially larger than this. Given the odd nature of its optical spectrum, we feel that 2MASS $\mathrm{J} 1949+6222$ is not in the same class of object as the other blue L dwarfs found during our survey and is worthy of additional follow-up. 


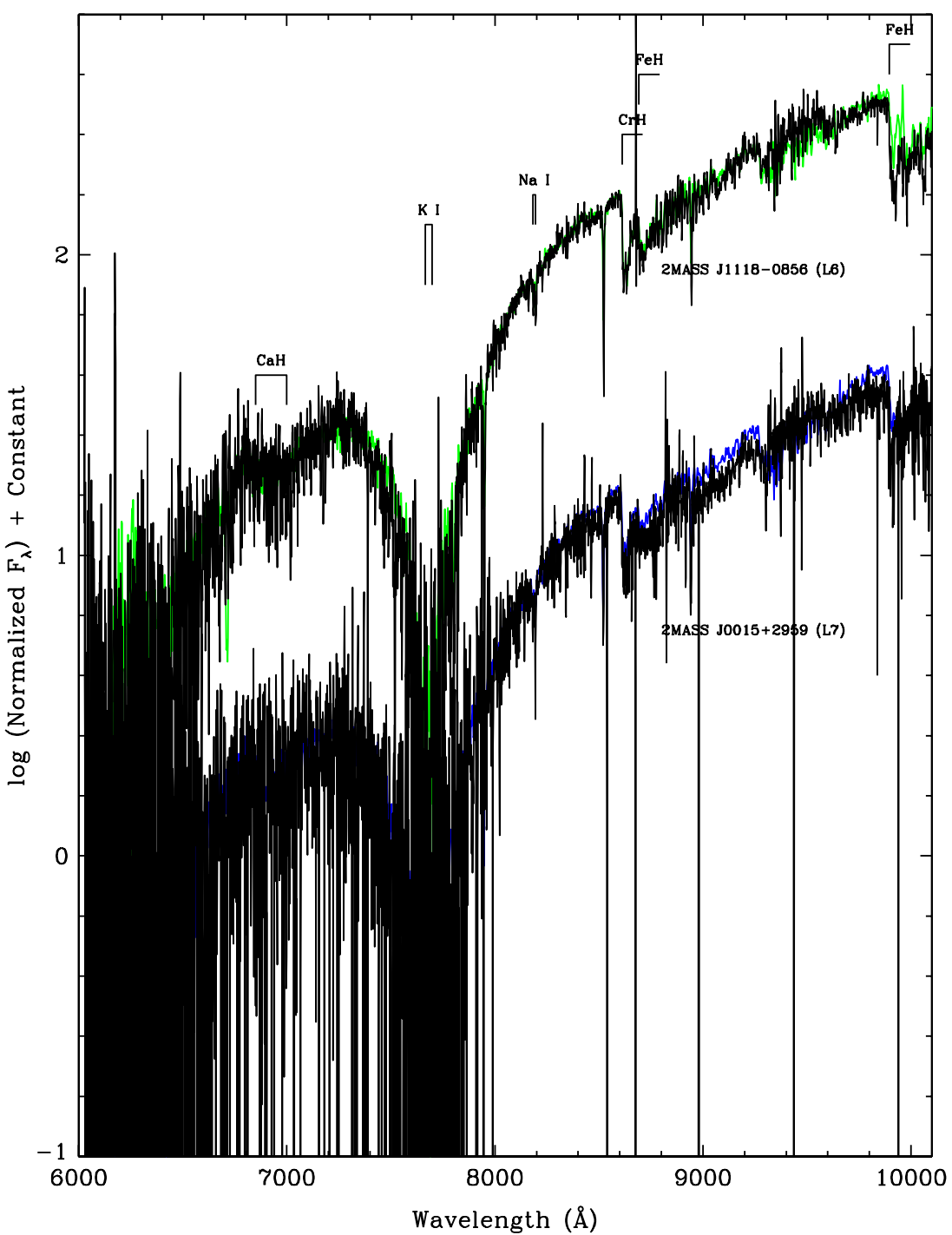

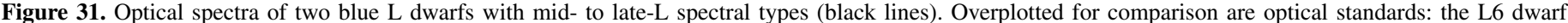

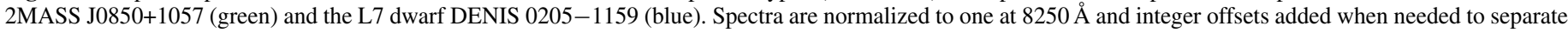
the spectra vertically.

(A color version of this figure is available in the online journal.)

2MASS J21513979+3402444. The near-infrared spectrum of this object (Figure 34) best fits the L7 near-infrared standard. At longer wavelengths, the overall continuum is suppressed relative to that same standard, earning this object a "blue L" designation.

A full list of known blue L dwarfs is given in Table 10. For the new discoveries, we estimate a distance using the 2MASS $J$-band magnitude and the predicted absolute $J$-band magnitude given by the optical spectral type, as given by the relation of Looper et al. (2008). (The near-infrared spectral type is used when an optical type is not available.) With this larger sample of blue $\mathrm{L}$ dwarfs we find a mean transverse velocity of $\sim 66 \mathrm{~km} \mathrm{~s}^{-1}$, which is somewhat lower than the number computed by Faherty et al. (2009) but still within their $1 \sigma$ errors. As stated earlier, the blue L dwarfs in this list probably represent a mixture of different types. Follow-up of suspected binaries such as SDSS J0805+4812 (Burgasser et al. 2007) should be done to eliminate objects that are extrinsically blue, leaving a purer sample of intrinsically blue L dwarfs for further study.

In Table 13, we compute radial velocities and total space motions for our blue L dwarfs. Discounting the two blue $\mathrm{L}$ dwarfs with large uncertainties in $V_{\tan }$, we find an average space motion of $141 \mathrm{~km} \mathrm{~s}^{-1}$, similar to the value found for the red $\mathrm{L}$ dwarfs but smaller than the value found for late-M and $\mathrm{L}$ subdwarfs. This raises the intriguing possibility that the red $\mathrm{L}$ dwarf and blue L dwarf phenomena are related, or at least occur in L dwarfs of similar age. It is even possible that these two phenomena occur in the same objects and that viewing angle determines the spectral appearance. This might be the case if clouds are not homogeneously distributed in latitude or if properties such as grain size and cloud thickness vary in latitude. In this scenario the red dwarf phenomenon would coincide with the less frequent case of pole-on viewing angle, and the blue L dwarf phenomenon would relate to the more frequent case of equator-on viewing angles. This hypothesis can be tested by measuring the rotational velocities of objects in the two samples to see if the red L dwarfs in general show smaller $V_{\text {rot }}$ values. Even then, the reason for the odd distribution of clouds - thicker or large-grained clouds at the poles and thinner or smaller-grained clouds near the equator-and its predilection for objects in this range of ages would have to be explained theoretically. Whether or not this hypothesis has any basis in reality, it is still possible to gather more observa- 


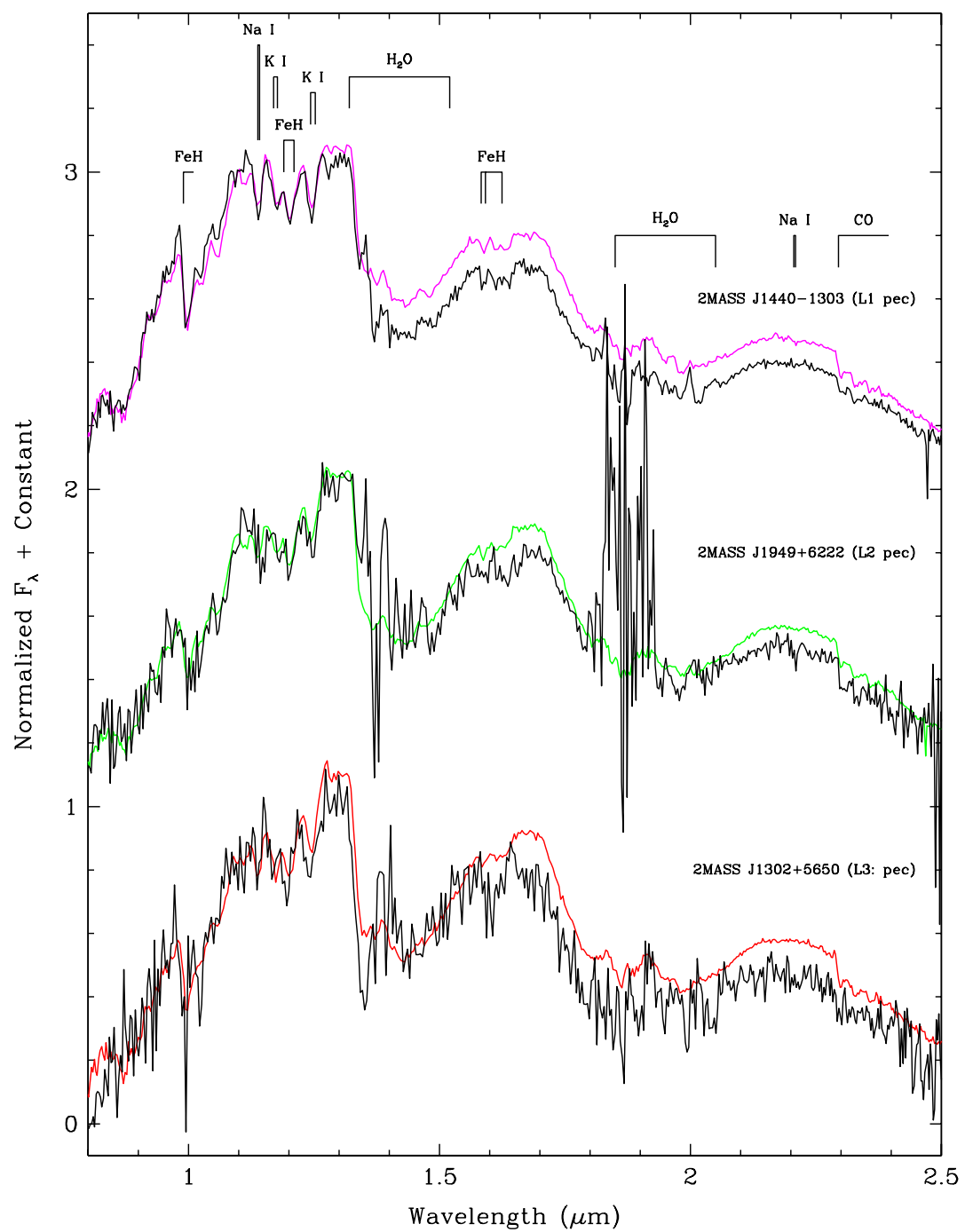

Figure 32. Near-infrared spectra of three blue L dwarf discoveries at early-L types (black lines). Overplotted for comparison are near-infrared standards: the L1 dwarf 2MASS J2130-0845 (magenta), L2 dwarf Kelu-1 (green), and L3 dwarf 2MASS J1506+1321 (red). Spectra are normalized to one at $1.28 \mu \mathrm{m}$ and integer offsets added when needed to separate the spectra vertically.

(A color version of this figure is available in the online journal.)

tional clues even on the red $\mathrm{L}$ and blue $\mathrm{L}$ samples currently identified.

\section{5. $T$ Dwarfs}

$\mathrm{T}$ dwarfs share the same locus as hotter stars and $\mathrm{L}$ dwarfs in the standard $J-H$ versus $H-K_{s}$ diagram. In particular, early$\mathrm{T}$ dwarfs are extremely difficult to identify in 2MASS because they have near-infrared colors similar to $\mathrm{M}$ dwarfs, which are detected in far greater numbers since they are visible to much greater distances. With our proper motion survey, however, we have been able to identify $\mathrm{T}$ dwarfs regardless of color selection by using kinematic criteria.

As expected, our survey recovered previously identified, later T dwarfs such as the T8 dwarf 2MASS J09393548-2448279, one of the coolest dwarfs currently known (Burgasser et al. 2008). Our survey also uncovered some previously unidentified early-T dwarfs (Table 11). Looper et al. (2007) has already discussed three of these discoveries-2MASS J11061191+2754215 (T2.5), 2MASS J13243553+6358281 (T2 pec), and 2MASS J14044948-3159330 (T3). A fourth object, 2MASS J15111466+0607431 (T2 pec), was independently identified by Chiu et al. (2006) using data from the SDSS. A fifth object is presented here for the first time,

$2 M A S S J 21265916+7617440$. The optical spectrum is shown in Figure 35. The overall continuum shape matches that of the L7 optical standard although there are difference in bandstrengths of some molecular features. The main disagreement between the two spectra is in the strengths of the $\mathrm{FeH}$ and $\mathrm{CrH}$ bands, which are weaker in 2MASS J2126+7617. (The spectrum of the optical standard, unlike that of 2MASS J2126+7617, has not been corrected for telluric absorption and thus does not match well in the 9300-9850 ̊ water band.) Despite the late-L dwarf classification in the optical, the near-infrared spectrum (Figure 36) shows methane absorption features at both $H$ and $K$ bands and warrants a T spectral type. A T0 standard spectrum best matches the near-infrared features, but there are clear discrepancies. In 2MASS J2126+7617, the FeH band at $9796 \AA$ is too strong, there is excess flux near the $1.28 \mu \mathrm{m}$ peak at $J$ band, the $H$-band methane feature is too strong, and the $K$-band portion of the spectrum is slightly suppressed.

These features can be reproduced if this object is assumed to be an L dwarf $+\mathrm{T}$ dwarf binary. Fitting to the suite of single spectra and synthetic composite spectra in Burgasser 


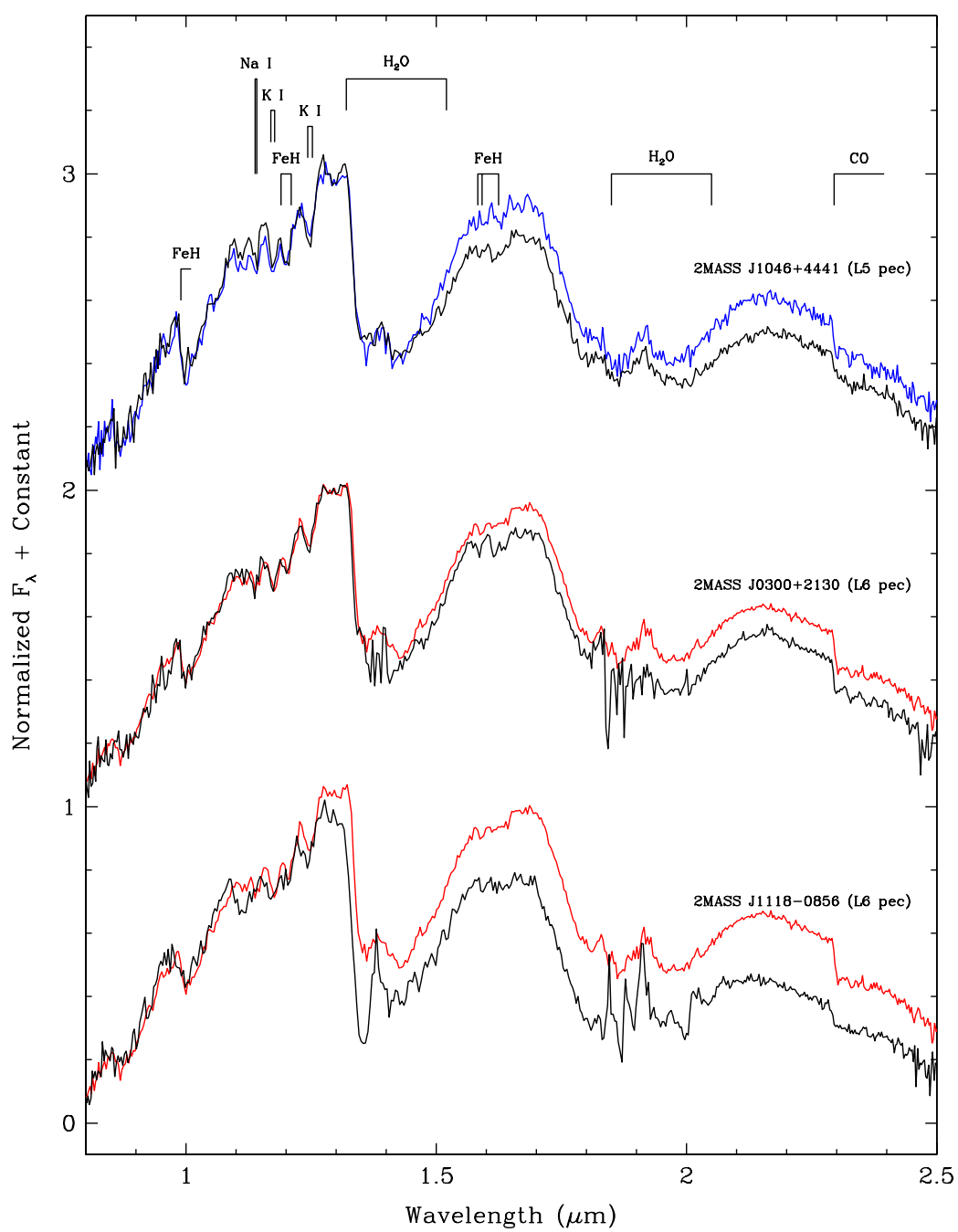

Figure 33. Near-infrared spectra of three blue L dwarf discoveries at mid-L types (black lines). Overplotted for comparison are near-infrared standards: the L5 dwarf 2MASS J0835+1953 (blue) and L6 dwarf 2MASS J1010-0406 (red). Spectra are normalized to one at $1.28 \mu \mathrm{m}$ and integer offsets added when needed to separate the spectra vertically.

(A color version of this figure is available in the online journal.)

et al. (2010) and using a modified $\chi^{2}$ statistic (called $\mathrm{G}_{k}$, from Cushing et al. 2008), we find that the best binary fit is a statistically significant better match than the best fit to a single source (see Equation (4) in Burgasser et al. 2010). Fits to spectroscopic composites whose components are scaled using the Looper et al. (2008) absolute magnitude versus spectral type relation suggest that the binary is comprised of an L7 and a T3.5 dwarf (see Figure 37), with an error of just under one spectral subclass for each component. Given that the secondary of 2MASS J2126+7617 is expected to fall very near the spectral type where the $J$-band bump is located (i.e., the local maximum in the $M_{J}$ versus spectral type relation for $\mathrm{T}$ dwarfs), this potential binary could be as important a probe of atmospheric flux distribution over the L/T transition as the binary 2MASS J1401-3159 (Looper et al. 2008), also discovered during our proper motion survey.

\subsection{Other Interesting Objects}

Other noteworthy objects not meeting the categories above are discussed here and listed in Table 12.

2MASS J04205430-7327392. This object is detected in the 2MASS $1 \mathrm{X}$ data at $J=13.159 \pm 0.024 \mathrm{mag}$ and $K_{s}=$
$10.203 \pm 0.021 \mathrm{mag}$. Curiously, this object is invisible in the 2MASS 6X data, which of course probe to much deeper limits $\left(\mathrm{S} / \mathrm{N}=10\right.$ limits of $J \approx 17.3 \mathrm{mag}$ and $K_{s} \approx 15.7 \mathrm{mag}$; Cutri et al. 2003). Given the extremely red color $\left(J-K_{s}=\right.$ $2.956 \pm 0.032 \mathrm{mag}$ ), presumed photometric (as opposed to astrometric) variability, and sky location, we believe this object may be a nova in the outskirts of the Large Magellanic Cloud.

2MASS J09073735-1457036. Like the previous object, this source is clearly visible in the $1 \mathrm{X}$ data, in this case with $\mathrm{J}=$ $15.322 \pm 0045 \mathrm{mag}$ and $K_{s}=14.847 \pm 0.149$, but completely disappears in the $6 \mathrm{X}$ data. Because of its blue color $\left(J-K_{s}=\right.$ $0.475 \pm 0.156$ ), it was observed as a candidate $\mathrm{T}$ dwarf in the survey of Burgasser et al. (2002) and was not found in deeper infrared images obtained on 2000 May 20 (UT). The 1X observation may have been of a previously unknown asteroid, uncataloged because this observation lies a full $30^{\circ}$ off the ecliptic.

2MASS J16002647-2456424. The near-infrared spectrum of this object (Figure 38) shows a morphology most like an M7, except that the 0.6-1.0 $\mu \mathrm{m}$ slope is steeper and the depth of the $\mathrm{H}_{2} \mathrm{O}$ band near $1.5 \mu \mathrm{m}$ is deeper than in a normal M7, the latter giving the $H$ band a triangular shape. This $H$-band morphology 
No. 1,2010

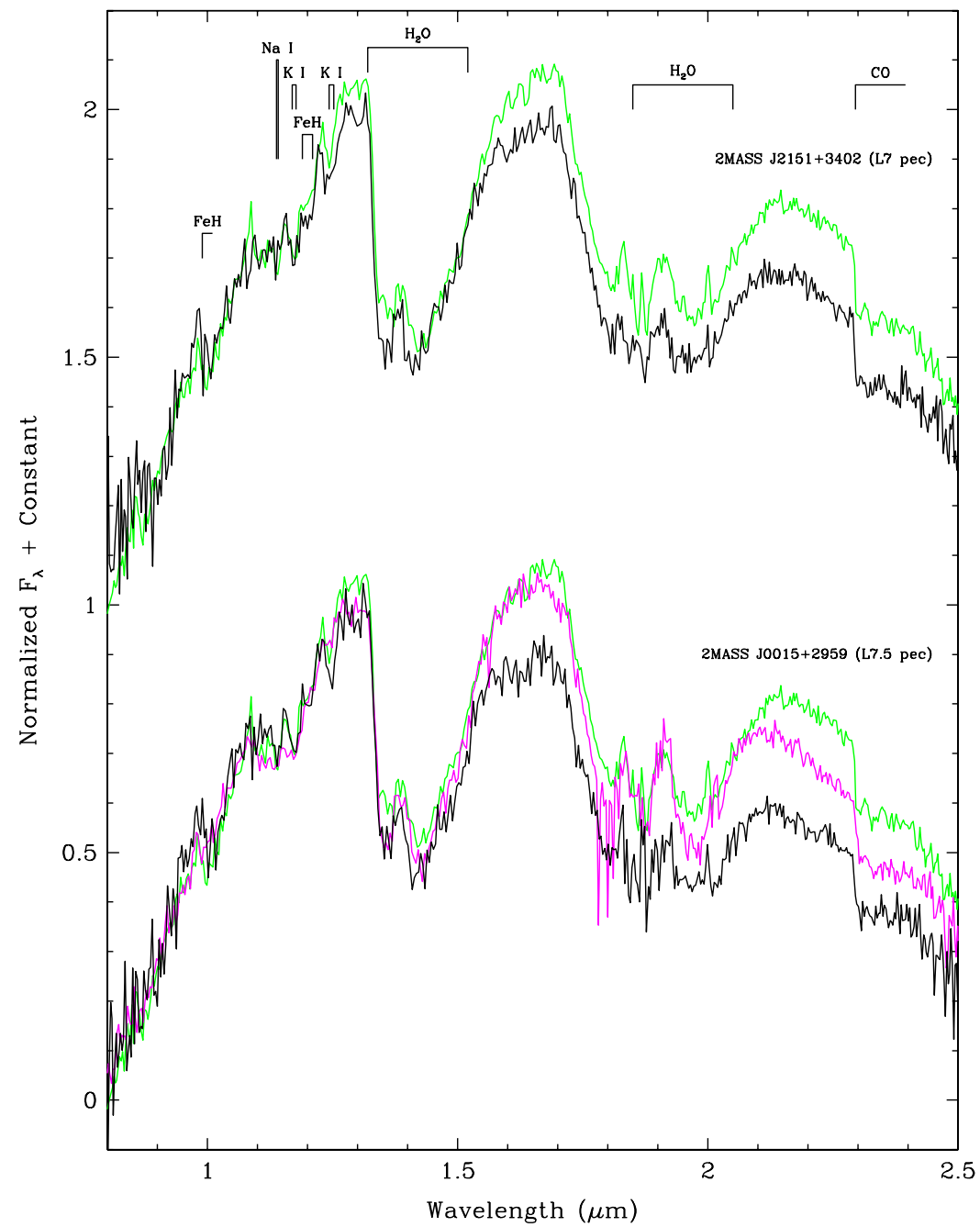

Figure 34. Near-infrared spectra of two blue L dwarf discoveries at late-L types (black lines). Overplotted for comparison are near-infrared standards: the L7 dwarf 2MASS J0103+1935 (green) and L8 dwarf 2MASS J1632+1904 (magenta). Spectra are normalized to one at $1.28 \mu \mathrm{m}$ and integer offsets added when needed to separate the spectra vertically.

(A color version of this figure is available in the online journal.)

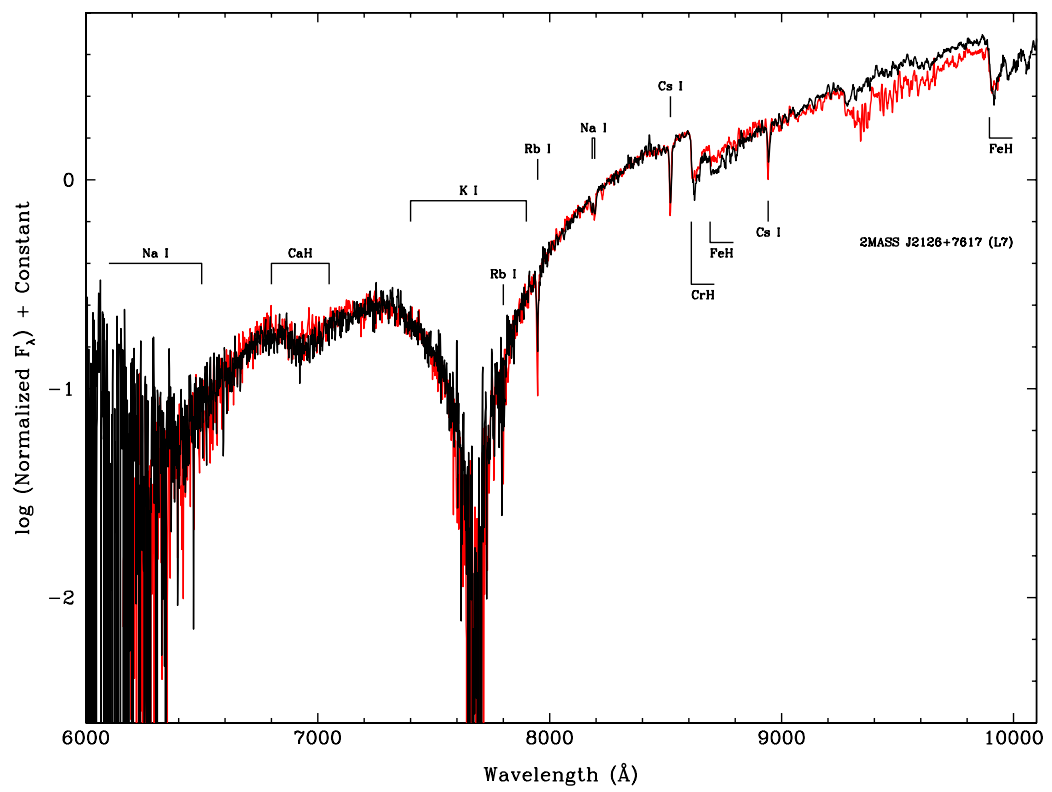

Figure 35. Subaru-FOCAS data for 2MASS J2126+7617 (black line) overplotted with the optical L7 standard DENIS J0205-1159 (red). Spectra are normalized to one at $8250 \mathrm{~A}$.

(A color version of this figure is available in the online journal.) 


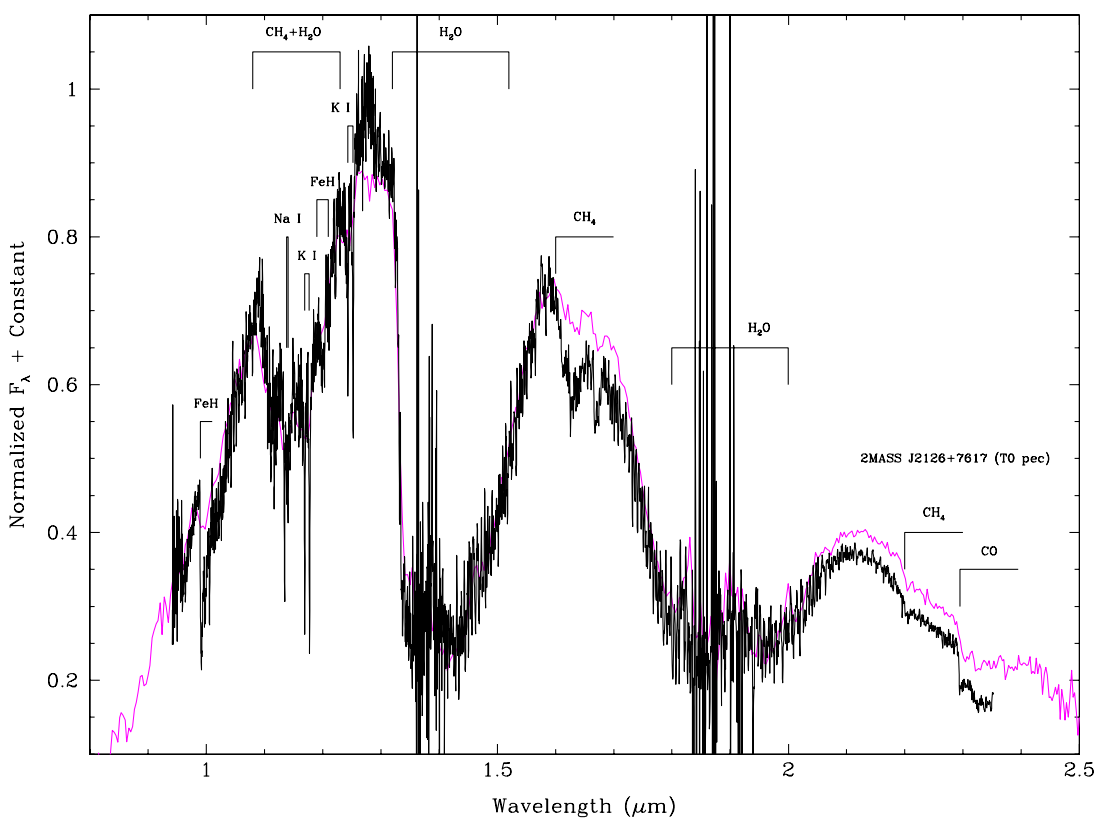

Figure 36. Keck-NIRSPEC spectrum of 2MASS J2126+7617, normalized to one at $1.28 \mu \mathrm{m}$ (black). Overplotted for comparison is the spectrum of the near-infrared T0 standard SDSS J1207+0244 (magenta), normalized to provide the best fit to the water bands of 2MASS J2126+7617.

(A color version of this figure is available in the online journal.)

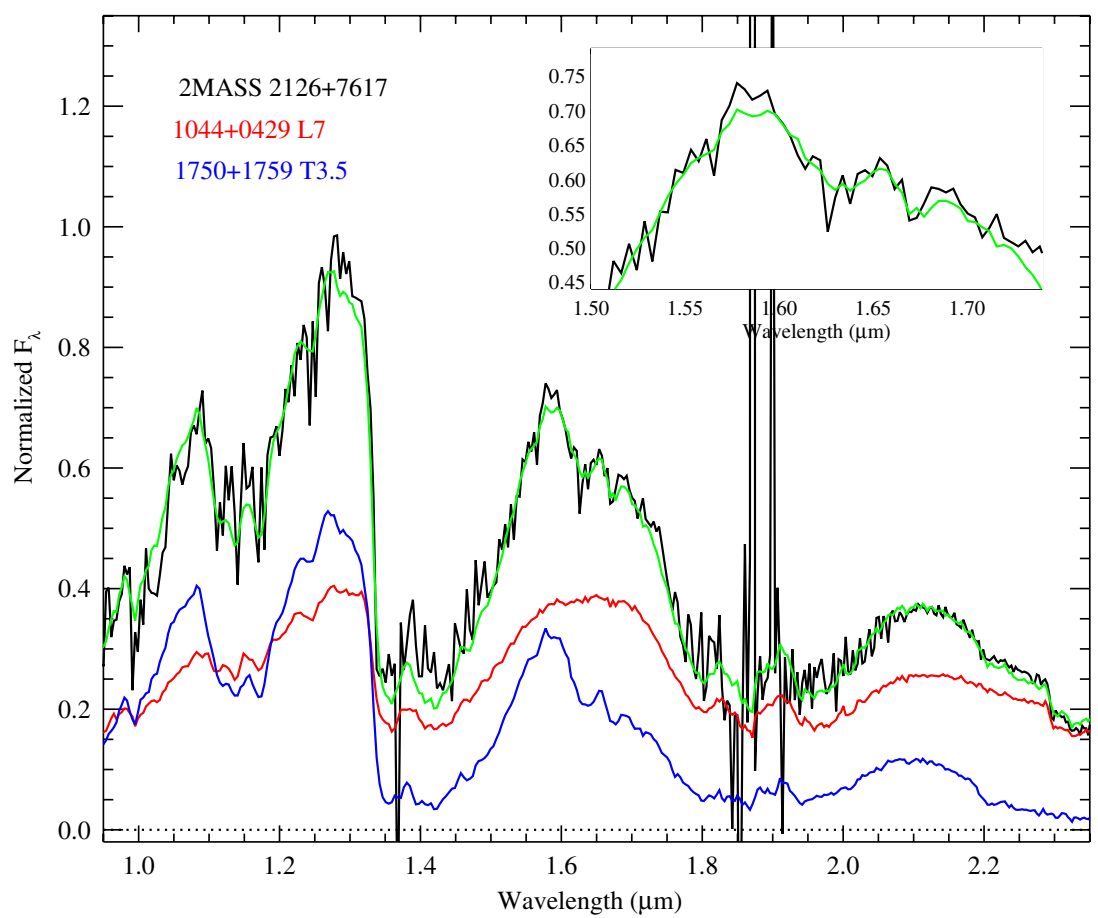

Figure 37. Spectral template of the best-fit binary (green line) compared to the near-infrared spectrum of 2MASS J2126+7617 (black line). Data for this source have been smoothed with a Gaussian kernel to match the resolution of the SpeX templates $(\lambda / \Delta \lambda \approx 120)$, and normalized in the $1.2-1.3 \mu \mathrm{m}$ window. The binary template is scaled to minimize its $\chi^{2}$ deviations. The primary (the L7 2MASS J1044+0429, red line) and secondary components (the T3.5 SDSS J1750+1759, blue line) of the best-fitting binary template are scaled according to their contribution to the combined-light template, based on the Looper et al. (2008) $M_{K_{s}} /$ spectral type relation. Inset box shows a close-up of the $1.5-1.75 \mu \mathrm{m}$ region.

(A color version of this figure is available in the online journal.)

is also seen in low-gravity objects, but this spectrum does not clearly exhibit other low-gravity features. We have no optical spectrum of this object.

2MASS J18284076+1229207. The near-infrared spectrum of this object (Figure 38) shows the same characteristics as the near-infrared spectrum of 2MASS J1600-2456 and is also best typed with a normal M7. In the case of 2MASS J1828+1229, we have an optical spectrum (not shown) which looks like a normal M8 dwarf.

2MASS J21403907+3655563. The near-infrared spectrum of this object (Figure 38 ) best fits the M8 standard over the $J$ band. At longer wavelengths the spectrum has excess flux relative to the M8 standard. This object may be a late-M analog to the "red L" dwarfs. 


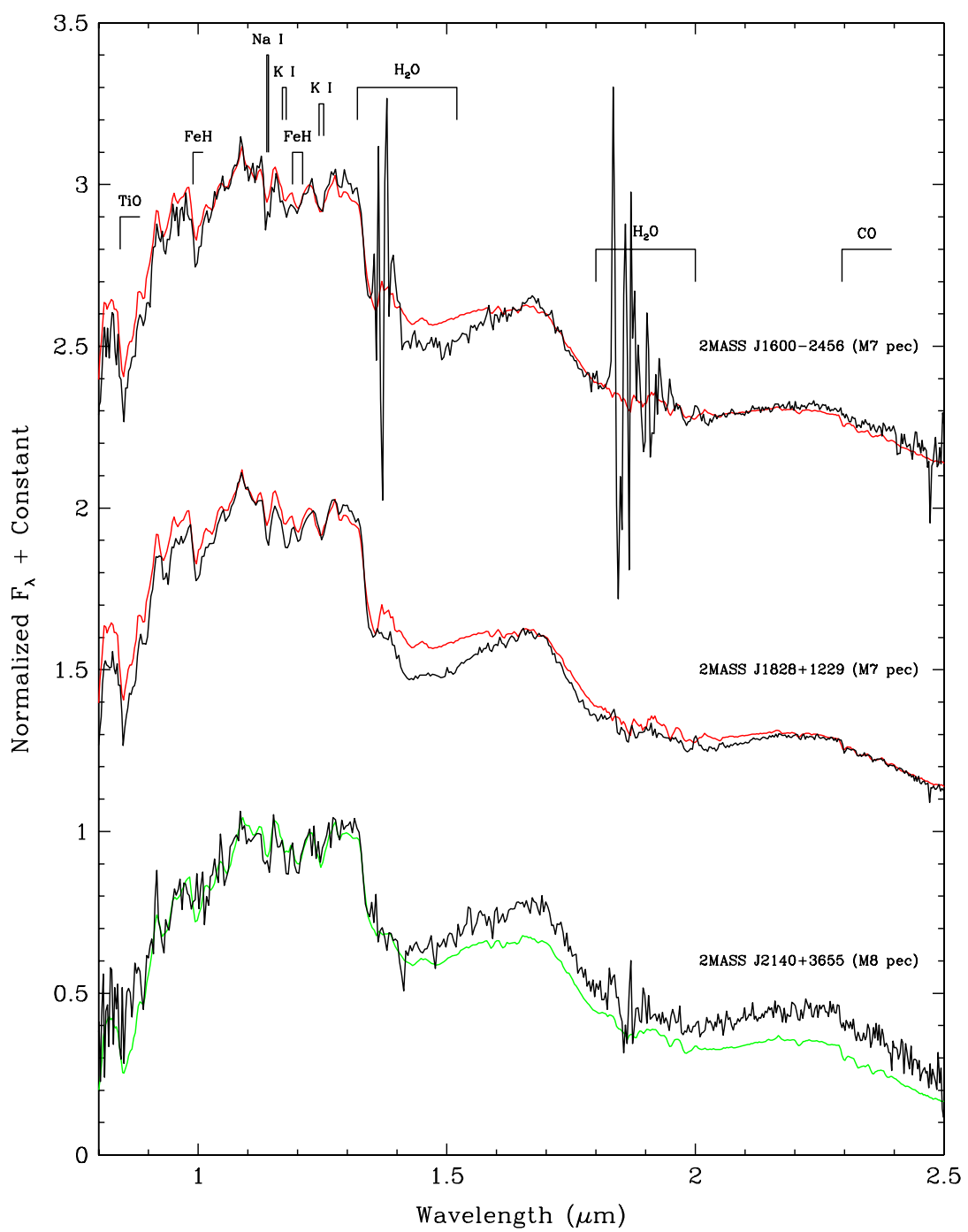

Figure 38. Near-infrared spectra of three slightly unusual spectra (black lines) that do not fit any of the other categories. Overplotted for comparison are spectra of the near-infrared M7 standard vB 8 (red) and M8 standard vB 10 (green). Spectra are normalized to one at $1.28 \mu \mathrm{m}$ and integer offsets added when needed to separate the spectra vertically.

(A color version of this figure is available in the online journal.)

\section{PROSPECTS FOR FUTURE BROWN DWARF SURVEYS}

Having now used proper motion to identify interesting objects that were not easily selected via their 2MASS colors alone, we look toward other surveys that will be able to extend this search. An extension in this case can be either to the $90 \%$ of the sky the 2MASS Proper Motion Survey was not able to reach or to even fainter magnitudes or longer wavelengths than probed here.

Two ongoing surveys that have both near-infrared sensitivity and multi-epoch coverage are the Large Area Survey of the United Kingdom Infrared Deep Sky Survey (UKIDSS-LAS) and surveys being done by the Visible and Infrared Survey Telescope for Astronomy (VISTA). The multi-epoch aspect of the UKIDSS-LAS will cover a similar area as the 2MASS Proper Motion Survey (4000 $\left.\mathrm{deg}^{2}\right)$, although in a single filter, but the sensitivity will be considerably deeper $(J \approx 20.0 \mathrm{mag}$; see http://www.ukidss.org/surveys/surveys.html) and the time baseline may be up to seven years. These areas also cover regions probed deeply by the SDSS at optical wavelengths; for objects in common to both surveys, these data will provide an even longer time baseline. VISTA (http://www.vista.ac.uk) has several surveys planned, the largest of which, the VISTA Hemisphere Survey (VHS), will cover the entire hemisphere in two near-infrared bands approximately 4 mag deeper than 2MASS. Three others (UltraVISTA, the VISTA Magellanic Survey, and VISTA Variables in the Via Lactea), though much smaller in extent, will have multi-epoch coverage.

Three other large-area surveys, all optically based, will enable astronomers to study the entire sky repeatedly in the time domain. The ongoing Panoramic Survey Telescope and Rapid Response System (Pan-STARRS; http://www.pan-starrs.ifa.hawaii.edu) plans to reach a cadence of $6000 \mathrm{deg}^{2}$ per night, surveying the entire sky (as seen from Hawaii, so $\sim 30,000 \mathrm{deg}^{2}$ ) over and over again in five or six bands, the longest wavelength of which will be either the $z$ - or $y$-band. The SkyMapper Telescope (Southern Sky Survey, aka S3; Keller et al. 2007) aims to observe all $\sim 20,000 \mathrm{deg}^{2}$ south of the celestial equator at $u, v, g, r, i$, and $z$ bands with cadences ranging from hours to years. The LSST (http://www.lsst.org) will cover over 20,000 $\mathrm{deg}^{2}$ of the Southern Hemisphere in six bands, covering the entire area roughly 1000 times during the course of the 10 year survey. Its longest wavelength filters will be $z$ - and $y$ bands. Even though based in the optical, all three of 


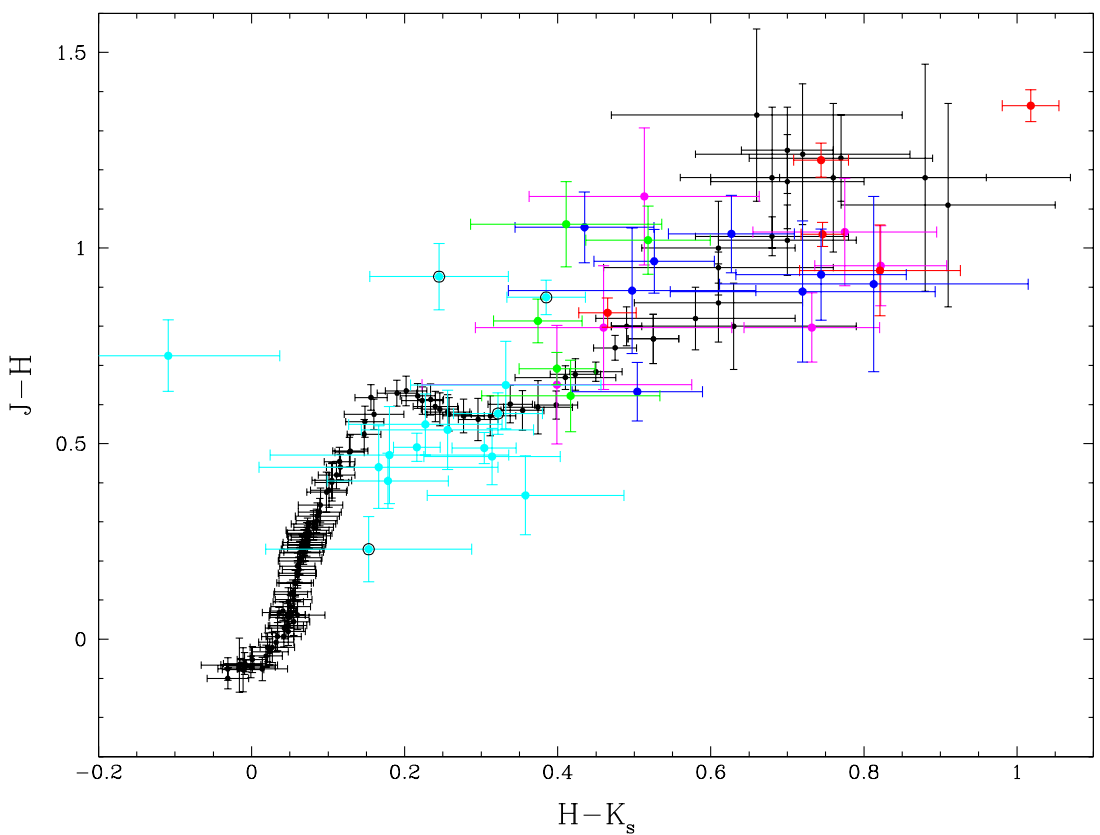

Figure 39. 2MASS $J-H$ vs. $H-K s$ color-color diagram. Black points represent averaged colors from the 2MASS All-Sky Point Source Catalog for dwarfs ranging in type from B2 (lower left) through L8 (upper right), sampled at every half spectral subclass. Objects from Tables 5, 6, 7, 9, and 11 are shown with colored points. Low-gravity (young) $\mathrm{M}$ and $\mathrm{L}$ dwarfs are shown in magenta, red L dwarf discoveries are in red, M and L subdwarfs are in cyan (with the sdM9.5, sdL1, sdL7, and sdL8 further highlighted with black open circles), blue $\mathrm{L}$ dwarf discoveries in blue, and $\mathrm{T}$ dwarf discoveries in green.

(A color version of this figure is available in the online journal.)

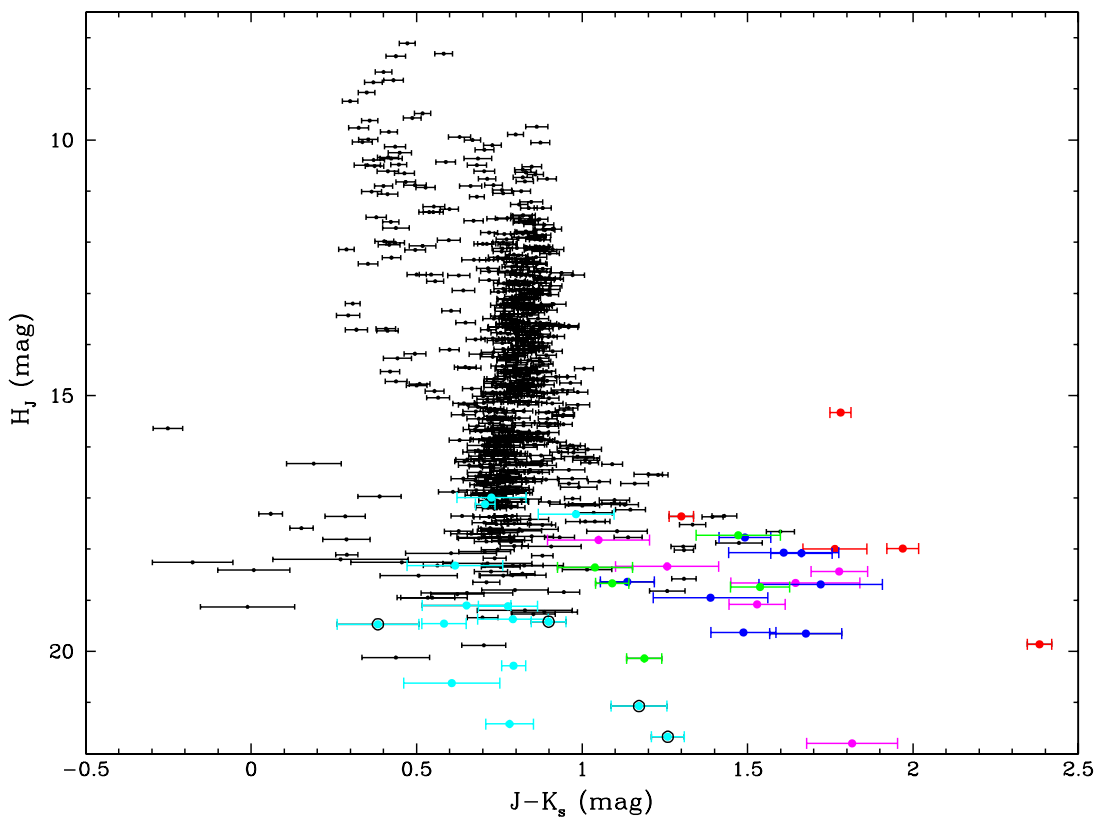

Figure 40. Blow-up of the top panel of Figure 7. Color coding is the same as that in Figure 39.

(A color version of this figure is available in the online journal.)

these surveys will probe deeply enough at $z$ and/or $y$ to canvass the Solar Neighborhood for $\mathrm{L}$ and $\mathrm{T}$ dwarfs.

Figure 39 shows the standard near-infrared color-color diagram with our most interesting discoveries from the 2MASS Proper Motion Survey color coded. With a notable exception or two-such as the extremely red color for 2MASS $\mathrm{J} 2148+4003$ - most of these objects fall in the same locus as normal late-M and $\mathrm{L}$ dwarfs. $\mathrm{M}$ subdwarfs, however, by virtue of their bluer $J-H$ and $H-K_{s}$ colors, fall below the locus of $\mathrm{K}$ and $\mathrm{M}$ dwarfs on this diagram and could be selected by the improved, very accurate colors of UKIDSS and VISTA alone.
Our late-M and $\mathrm{L}$ subdwarfs show a wider range of colors. Sometimes these objects are identifiable from their colors alone but sometimes (as in the case of 2MASS J1756+2815) they are indistinguishable from much more common M dwarfs.

With the addition of proper motion information, plots like Figure 40 can be used to find interesting objects. In this plot, the subdwarfs again distinguish themselves by being bluer than the other late-type dwarfs and also by having very large reduced proper motions (although this is partly a selection effect since we used this plot to prioritize candidates for follow-up). Lowgravity $\mathrm{M}$ and $\mathrm{L}$ dwarfs, red $\mathrm{L}$ dwarfs, blue $\mathrm{L}$ dwarfs, and 


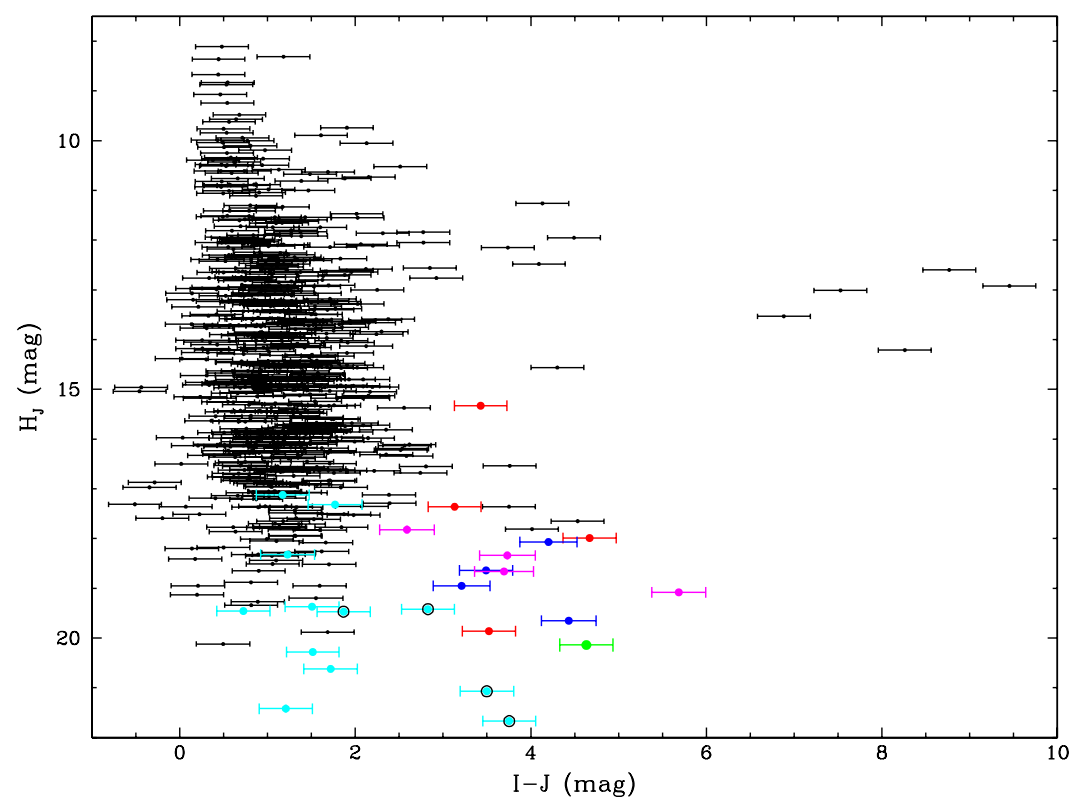

Figure 41. Reduced proper motion at $J$-band plotted against $I-J$ color. Black points represent those 2MASS proper motion objects whose motions are at least five times the astrometric error. Objects from Tables 6, 7, and 11 are shown with colored points. Red L dwarf discoveries are shown in red, M and L subdwarfs are in cyan, and $\mathrm{T}$ dwarf discoveries in green. (None of the low-gravity or blue L dwarfs were detected in the USNO-B $I$ band and hence cannot be displayed.) For a few of the brighter red L dwarfs, L subdwarfs, and T dwarfs, $I-J$ colors limits are shown as triangles. In these cases, an $I$-band limit of 18.5 mag is assumed. (Note that objects with $I-J>6$ have erroneous $I$-band magnitudes from the USNO-B.)

early-T dwarfs share the same locus on this diagram and cannot be easily distinguished from one another. Near-infrared surveys like UKIDSS-LAS and the various VISTA surveys, even with a proper motion component, will have trouble distinguishing these objects from the sea of normal L dwarfs.

If we use the 2MASS $J$ band as a proxy for $y$, we can envision what a similar reduced proper motion diagram might look like for Pan-STARRS or LSST. In Figure 41, we plot $I-J$ color as a proxy for $i-y$. As with Figure 40, subdwarfs tend to separate cleanly from the other objects, but other interesting types again fall in the same color locus. It is almost certainly the case that in the absence of spectroscopic follow-up, the only hope of distinguishing these objects is to combine photometry from large-area surveys at different wavelengths and look for other optical/infrared colors that might help segregate the types. Color combinations that come closest to the way these objects are defined may, of course, have the best degree of success. An optical color such as $i-z$ that can be used as a proxy for optical spectral type (Hawley et al. 2002, Knapp et al. 2004) can be plotted against $J-K_{s}$, in much the same way that Figure 14 of Kirkpatrick et al. (2008) can separate red L dwarfs and lowgravity objects from blue L dwarfs and low-metallicity objects.

Finally, there is one new survey that-by virtue of its allsky coverage, multi-epoch data (though only with an epoch difference of six months), and sensitivity at wavelengths where brown dwarfs have their peak flux - has the best chance of canvassing the immediate Solar Neighborhood completely for all M, L, and T dwarf exotica. The Wide-field Infrared Survey Explorer (WISE; http://wise.ssl.berkeley.edu) was launched on 2009 Dec 14 and promises to give a view of local brown dwarfs like no other previous study. Combining its four bands of data (centered near 3.4, 4.6, 12, and $22 \mu \mathrm{m}$ ) with other existing surveys will aid greatly in our ability to distinguish these exotica from the more mundane $\mathrm{M}, \mathrm{L}$, and $\mathrm{T}$ dwarfs.

We thank John Stauffer and Maria Morales-Calderon for giving us some telescope time in exchange for instrument expertise on 2005 December 9 (UT) at Keck-II. We thank Lee Rottler for assistance in installing and troubleshooting various science software packages in support of this paper. We thank Francesca Colonnese for help with intermediate versions of the tables for the $1 \mathrm{X}-6 \mathrm{X}$ survey and Rob Simcoe for taking some of the Magellan-Clay observations. We are indebted to the support staff at each of the observatories we used; their expertise was instrumental in making this survey a success. We acknowledge use of The Digitized Sky Surveys, which were produced at the Space Telescope Science Institute under U.S. Government grant NAG W-2166. The images of these surveys are based on photographic data obtained using the Oschin Schmidt Telescope on Palomar Mountain and the UK Schmidt Telescope. This publication makes use of data products from the Two Micron All Sky Survey, which is a joint project of the University of Massachusetts and the Infrared Processing and Analysis Center/California Institute of Technology, funded by the National Aeronautics and Space Administration and the National Science Foundation. This research has made use of the NASA/IPAC Infrared Science Archive, which is operated by the Jet Propulsion Laboratory, California Institute of Technology, under contract with the National Aeronautics and Space Administration. Our research has benefitted from the M, L, and T dwarf compendium housed at DwarfArchives.org, whose server was funded by a NASA Small Research Grant, administered by the American Astronomical Society. We are also indebted to the SIMBAD database, operated at CDS, Strasbourg, France. We further recognize and acknowledge the very significant cultural role and reverence that the summit of Mauna Kea has always had within the indigenous Hawaiian community. We are most fortunate to have the opportunity to conduct observations from this mountain.

\section{REFERENCES}

Allington-Smith, J., et al. 1994, PASP, 106, 983

Barnard, E. E. 1916, AJ, 29, 181

Benedict, G. F., et al. 1999, AJ, 118, 1086 
Bochanski, J. J., et al. 2009, PASP, 121, 1409

Borysow, A., Jorgensen, U. G., \& Zheng, C. 1997, A\&A, 324, 185

Bowler, B. P., Liu, M. C., \& Dupuy, T. J. 2010, ApJ, 710, 45

Burgasser, A. J. 2004, ApJ, 614, L73

Burgasser, A. J. 2007, AJ, 134, 1330

Burgasser, A. J., Cruz, K. L., Cushing, M., Gelino, C. R., Looper, D. L., Faherty, J. K., Kirkpatrick, J. D., \& Reid, I. N. 2010, ApJ, 710, 1142

Burgasser, A. J., Cruz, K. L., \& Kirkpatrick, J. D. 2007, ApJ, 657, 494

Burgasser, A. J., Geballe, T. R., Leggett, S. K., Kirkpatrick, J. D., \& Golimowski, D. A. 2006, ApJ, 637, 1067

Burgasser, A. J., Kirkpatrick, J. D., Liebert, J., \& Burrows, A. 2003, ApJ, 594, 510

Burgasser, A. J., Lépine, S., Lodieu, N., Scholz, R. D., Delorme, P., Jao, W. C. Swift, B. J., \& Cushing, M. C. 2009, in AIP Conf. Ser. 1094, Cool Stars, Stellar Systems and the Sun, ed. E. Stempels (Melville, NY: AIP), 242

Burgasser, A. J., Looper, D. L., Kirkpatrick, J. D., Cruz, K. L., \& Swift, B. J. 2008, ApJ, 674, 451

Burgasser, A. J., Tinney, C. G., Cushing, M. C., Saumon, D., Marley, M. S., Bennett, C. S., \& Kirkpatrick, J. D. 2008, ApJ, 689, L53

Burgasser, A. J., Vrba, F. J., Lépine, S., Munn, J. A., Luginbuhl, C. B., Henden, A. A., Guetter, H. H., \& Canzian, B. C. 2008, ApJ, 672, 1159

Burgasser, A. J., et al. 2002, ApJ, 564, 421

Burgasser, A. J., et al. 2003, ApJ, 592, 1186

Chiu, K., Fan, X., Leggett, S. K., Golimowski, D. A., Zheng, W., Geballe, T. R., Schneider, D. P., \& Brinkmann, J. 2006, AJ, 131, 2722

Cruz, K. L., Kirkpatrick, J. D., \& Burgasser, A. J. 2009, AJ, 137, 3345

Cruz, K. L., Reid, I. N., Liebert, J., Kirkpatrick, J. D., \& Lowrance, P. J. 2003, AJ, 126,2421

Cushing, M. C., Looper, D., Burgasser, A. J., Kirkpatrick, J. D., Faherty, J., Cruz, K. L., Sweet, A., \& Sanderson, R. E. 2009, ApJ, 696, 986

Cushing, M. C., \& Vacca, W. D. 2006, AJ, 131, 1797

Cushing, M. C., Vacca, W. D., \& Rayner, J. T. 2004, PASP, 116, 362

Cushing, M. C., et al. 2006, ApJ, 648, 614

Cushing, M. C., et al. 2008, ApJ, 678, 1372

Cutri, R. M., et al. 2003, The IRSA 2MASS All-Sky Point Source Catalog, NASA/IPAC Infrared Science Archive, http://irsa.ipac.caltech.edu/ applications/Gator/

Dahn, C. C., et al. 2002, AJ, 124, 1170

Deacon, N. R., \& Hambly, N. C. 2007, A\&A, 468, 163

Deacon, N. R., Hambly, N. C., \& Cooke, J. A. 2005, A\&A, 435, 363

Deacon, N. R., Hambly, N. C., King, R. R., \& McCaughrean, M. J. 2009, MNRAS, 394, 857

Faber, S. M., et al. 2003, Proc. SPIE, 4841, 1657

Faherty, J. K., Burgasser, A. J., Cruz, K. L., Shara, M. M., Walter, F. M., \& Gelino, C. R. 2009, AJ, 137, 1

Finch, C. T., Henry, T. J., Subasavage, J. P., Jao, W.-C., \& Hambly, N. C. 2007, AJ, 133, 2898

Folkes, S. L., Pinfield, D. J., Kendall, T. R., \& Jones, H. R. A. 2007, MNRAS, 378,901

Geballe, T. R., et al. 2002, ApJ, 564, 466

Giclas, H. L., Burnham, R., Jr., \& Thomas, N. G. 1978, Lowell Obs. Bull., 8, 89

Giclas, H. L., Burnham, R., \& Thomas, N. G. 1971, The Lowell Proper Motion Survey (Flagstaff, AZ: Lowell Observatory)

Gizis, J. E. 1997, AJ, 113, 806

Gizis, J. E., Jao, W.-C., Subasavage, J. P., \& Henry, T. J. 2007, ApJ, 669, L45

Gizis, J. E., Monet, D. G., Reid, I. N., Kirkpatrick, J. D., Liebert, J., \& Williams, R. J. 2000, AJ, 120, 1085

Hamuy, M., Suntzeff, N. B., Heathcote, S. R., Walker, A. R., Gigoux, P., \& Phillips, M. M. 1994, PASP, 106, 566

Hawley, S.L., et al. 2002, AJ, 123, 3409

Hook, I. M., Jørgensen, I., Allington-Smith, J. R., Davies, R. L., Metcalfe, N., Murowinski, R. G., \& Crampton, D. 2004, PASP, 116, 425

Houck, J. R., et al. 2004, ApJS, 154, 18

Innes, R. T. A. 1915, Union Obs. Circ., No. 30

Ivezic, Z., Tyson, J. A., Allsman, R., Andrew, J., \& Angel, R. (for the LSST Collaboration) 2008, arXiv:0805.2366

Kaiser, N. 2004, Proc. SPIE, 5489, 11

Kashikawa, N., et al. 2002, PASJ, 54, 819

Keller, S. C., et al. 2007, PASA, 24, 1

Kelson, D. D. 2003, PASP, 115, 688

Kirkpatrick, J. D. 2005, ARA\&A, 43, 195

Kirkpatrick, J. D., Barman, T. S., Burgasser, A. J., McGovern, M. R., McLean, I. S., Tinney, C. G., \& Lowrance, P. J. 2006, ApJ, 639, 1120

Kirkpatrick, J. D., Henry, T. J., \& McCarthy, D. W., Jr. 1991, ApJS, 77, 417
Kirkpatrick, J. D., Henry, T. J., \& Simons, D. A. 1995, AJ, 109, 797

Kirkpatrick, J. D., \& McCarthy, D.W., Jr. 1994, AJ, 107, 333

Kirkpatrick, J. D., et al. 1999, ApJ, 519, 802

Kirkpatrick, J. D., et al. 2000, AJ, 120, 447

Kirkpatrick, J. D., et al. 2008, ApJ, 689, 1295

Knapp, G. R., et al. 2004, AJ, 127, 3553

Lawrence, A., et al. 2007, MNRAS, 379, 1599

Lépine, S. 2005, AJ, 130, 1247

Lépine, S. 2008, AJ, 135, 2177

Lépine, S., Rich, R. M., \& Shara, M. M. 2007, ApJ, 669, 1235

Lépine, S., Shara, M. M., \& Rich, R. M. 2002, AJ, 124, 1190

Lépine, S., Shara, M. M., \& Rich, R. M. 2003, AJ, 126, 921

Lodieu, N., Zapatero Osorio, M. R., Martín, E. L., Solano, E., \& Aberasturi, M. 2010, ApJ, 708, L107

Looper, D. L., Gelino, C. R., Bugasser, A. J., \& Kirkpatrick, J. D. 2008, ApJ, 685,1183

Looper, D. L., Kirkpatrick, J. D., \& Burgasser, A. J. 2007, AJ, 134, 1162

Looper, D. L., et al. 2008, ApJ, 686, 528

Luhman, K. L., Briceno, D., Rieke, G. H., \& Hartmann, L. 1998, ApJ, 493, 909

Luhman, K. L., Liebert, J., \& Rieke, G. H. 1997, ApJ, 489, L165

Luhman, K. L., Whitney, B. A., Meade, M. R., Babler, B. L., Indebetouw, R., Bracker, S., \& Churchwell, E. B. 2006, ApJ, 647, 1180

Luyten, W. J. 1925, ApJ, 62, 8

Luyten, W. J. 1979a, New Luyten Catalogue of Stars with Proper Motions Larger than Two Tenths of an Arcsecond, 2, 0

Luyten, W. J. 1979b, New Luyten Catalogue of Stars with Proper Motions Larger than Two Tenths of an Arcsecond, 1, 0

Luyten, W. J. 1980a, New Luyten Catalogue of Stars with Proper Motions Larger than Two Tenths of an Arcsecond, 4, 0

Luyten, W. J. 1980b, New Luyten Catalogue of Stars with Proper Motions Larger than Two Tenths of an Arcsecond, 3, 0

Marshall, J. L., et al. 2008, Proc. SPIE, 7014, 169

Massey, P., \& Gronwall, C. 1990, ApJ, 358, 344

McLean, I. S., Graham, J. R., Becklin, E. E., Figer, D. F., Larkin, J. E., Levenson, N. A., \& Teplitz, H. I. 2000, Proc. SPIE, 4008, 1048

McLean, I. S., McGovern, M. R., Burgasser, A. J., Kirkpatrick, J. D., Prato, L., \& Kim, S. S. 2003, ApJ, 596, 561

McLean, I. S., et al. 1998, Proc. SPIE, 3354, 566

Monet, D. G., et al. 2003, AJ, 125, 984

Oke, J. B., et al. 1995, PASP, 107, 375

Pokorny, R. S., Jones, H. R. A., Hambly, N. C., \& Pinfield, D. J. 2004, A\&A, 421, 763

Radigan, J., Lafrenière, D., Jayawardhana, R., \& Doyon, R. 2008, ApJ, 689 471

Rayner, J. T., Toomey, D. W., Onaka, P. M., Denault, A. J., Stahlberger, W. E., Vacca, W. D., Cushing, M. C., \& Wang, S. 2003, PASP, 115, 362

Reid, I. N., Burgasser, A. J., Cruz, K. L., Kirkpatrick, J. D., \& Gizis, J. E. 2001, AJ, 121, 1710

Reid, I. N., Cruz, K. L., Kirkpatrick, J. D., Allen, P. R., Mungall, F., Liebert, J., Lowrance, P., \& Sweet, A. 2008, AJ, 136, 1290

Reid, I. N., Hawley, S. L., \& Gizis, J. E. 1995, AJ, 110, 1838

Ross, F. E. 1926, AJ, 36, 124

Ruiz, M. T., Wischnjewsky, M., Rojo, P. M., \& Gonzalez, L. E. 2001, ApJS, 133,119

Schilbach, E., Röser, S., \& Scholz, R.-D. 2009, A\&A, 493, L27

Schmidt, S. J., West, A. A., Burgasser, A. J., Bochanski, J. J., \& Hawley, S. L. 2010, AJ, 139, 1045

Scholz, R.-D., Irwin, M., Ibata, R., Jahreiß, H., \& Malkov, O. Y. 2000, A\&A, 353,958

Scholz, R.-D., \& Meusinger, H. 2002, MNRAS, 336, L49

Sheppard, S. S., \& Cushing, M. C. 2009, AJ, 137, 304

Steele, I. A., \& Jameson, R. F. 1995, MNRAS, 272, 630

Teegarden, B. J., et al. 2003, ApJ, 589, L51

Torres, C. A. O., Quast, G. R., Melo, C. H. F., \& Sterzik, M. F. 2008, in Handbook of Star Forming Regions, Vol. II, ed. B. Reipurth (The Southern Sky ASP Monograph Publications, Vol. 5; San Francisco, CA: ASP), 757

Vacca, W. D., Cushing, M. C., \& Rayner, J. T. 2003, PASP, 115, 389

van Biesbroeck, G. 1944, AJ, 51, 61

van Biesbroeck, G. 1961, AJ, 66, 528

Vrba, F. J., et al. 2004, AJ, 127, 2948

Wolf, M. 1919, Veroeffentlichungen der Badischen Sternwarte zu Heidelberg, 7, 195

Wroblewski, H., \& Costa, E. 2001, A\&A, 367, 725

Zhang, Z. H., et al. 2009, A\&A, 497, 619

Zuckerman, B., \& Song, I. 2004, ARA\&A, 42, 685 